University of Wisconsin Milwaukee UWM Digital Commons

Theses and Dissertations

5-1-2014

\title{
Transverse Anderson Localization in Disordered Optical Fibers
}

Salman Karbasivalashani

University of Wisconsin-Milwaukee

Follow this and additional works at: https://dc.uwm.edu/etd

Part of the Electrical and Electronics Commons

\section{Recommended Citation}

Karbasivalashani, Salman, "Transverse Anderson Localization in Disordered Optical Fibers" (2014). Theses and Dissertations. 464. https://dc.uwm.edu/etd/464

This Dissertation is brought to you for free and open access by UWM Digital Commons. It has been accepted for inclusion in Theses and Dissertations by an authorized administrator of UWM Digital Commons. For more information, please contact open-access@uwm.edu. 


\title{
TRANSVERSE ANDERSON LOCALIZATION IN DISORDERED OPTICAL FIBERS
}

\author{
by \\ Salman Karbasivalashani \\ A Dissertation Submitted in \\ Partial Fulfillment of the \\ Requirements for the Degree of
}

Doctor of Philosophy

in Engineering

at

The University of Wisconsin-Milwaukee

May 2014 


\title{
ABSTRACT \\ TRANSVERSE ANDERSON LOCALIZATION IN DISORDERED OPTICAL FIBERS
}

\author{
by \\ Salman Karbasivalashani \\ The University of Wisconsin-Milwaukee, 2014 \\ Under the supervision of Prof. Arash Mafi
}

In any wave-guiding system, disorder and randomness in the wave propagation medium are considered as annoyances that result in wave scattering and inefficient wave transport. In contrast, in this work, the disorder is utilized in the transverse directions of an optical fiber for an effective light transport. The transversely disordered refractive index profile is invariant along the direction of propagation that results in transverse Anderson localization of light. A launched beam of light into the disordered fiber initially expands until it reaches the localization regime then propagates without further expansion in the transverse directions. A disordered polymer optical fiber composed of poly methyl methacrylate (PMMA) and poly styrene (PS) is designed using numerical simulations. The disordered polymer fiber is fabricated by drawing a preform of randomly mixed PMMA and PS strands. The light propagation in the disordered polymer fiber results in a localized beam radius that is comparable to the ones in the conventional optical fibers. The location of the transported beam at the output follows the location of the scanning beam at the input. In order to show the origin of transverse Anderson localization, the full vectorial modes of the disordered polymer optical fiber are calculated. The impacts of different design parameters on the light propagation in the disordered optical fibers are investigated. It is shown that the ultimate practical design is a disordered optical fiber that consists of glass and air sites with equal probability. The light propagation in a disordered glass optical fiber fabri- 
cated from porous glass with disordered air voids is studied as the first investigation of transverse Anderson localization in silica optical fibers. The non-uniform distribution of air voids in the glass host results in the wave localization in the regions with high fill-fraction of air voids.

The possibility of simultaneous multiple-beam propagation in the disordered polymer optical fiber is examined numerically and experimentally. The impact of macrobending on drifting the center of a propagating beam in the disordered polymer fiber is inspected. The macro-bending locally increases the refractive index difference between the disordered sites that results in a bend-insensitive wave propagation. The spatial multiplexing property of the disordered polymer fiber is utilized for high quality image transport. The quality of the transported images in the disordered polymer optical fiber is numerically and experimentally compared with the ones in the commercially available imaging fibers. The quality of the transported images is assessed using an effective objective evaluation technique. The impact of disorder on improving the image quality is specifically investigated by randomizing the radii of the cores in a periodic multicore fiber. 
(c) Copyright by Salman Karbasivalashani, 2014

All Rights Reserved 


\section{Dedicated to my beloved parents}

$$
\text { تقليم بِ يلهر و مادر عزيزم }
$$




\section{Contents}

1 Introduction $\quad 1$

1.1 Anderson localization . . . . . . . . . . . . . . 1

1.2 Transverse Anderson localization . . . . . . . . . . . . . 4

1.3 Motivation for this work and outline of dissertation . . . . . . . . 6

2 Finite Difference Beam Propagation Method 10

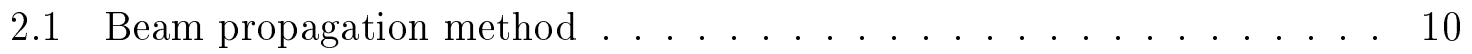

2.2 Runge-Kutta method for solving the first order differential equation . . 12

2.3 Stability of the Runge-Kutta method . . . . . . . . . . . . 13

2.3.1 Stability of forward Euler method . . . . . . . . . . . 13

2.3.2 Stability of $4^{t h}$ order Runge-Kutta method . . . . . . . . 15

2.4 Stability condition for a two-dimensional waveguide . . . . . . . . 19

2.5 Symplecticity and dissipation ................. 20

2.6 Transparent Boundary Condition for 1D waveguide . . . . . . . . 22

2.7 TBC for a $2 \mathrm{D}$ waveguide . . . . . . . . . . . . . 23 
2.8 Benchmarking the developed numerical code . . . . . . . . . . 25

3 Fabrication and characterization of the disordered Polymer Optical $\begin{array}{ll}\text { Fibers } & 27\end{array}$

3.1 Introduction . . . . . . . . . . . . . . . 27

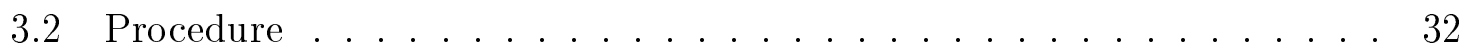

3.3 Representative results . . . . . . . . . . . . 36

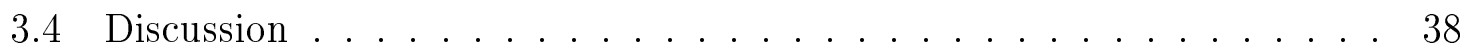

4 Transverse Anderson localization in the disordered polymer optical $\begin{array}{ll}\text { fiber } & 41\end{array}$

4.1 Introduction . . . . . . . . . . . . . . . . 41

4.2 Disordered polymer optical fibers . . . . . . . . . . . . . . 42

4.3 Comparing the experimental measurements with the theoretical calcu-

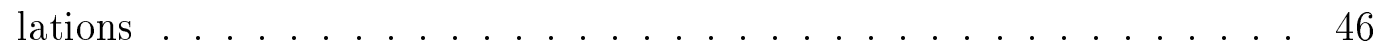

4.4 Localized modes in the disordered polymer optical fiber . . . . . . . . . 48

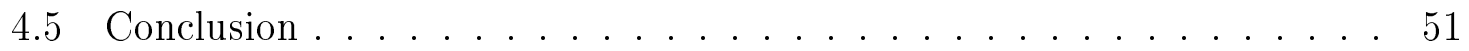

5 Effects of design parameters on the light localization in the Anderson $\begin{array}{ll}\text { localized optical fibers } & 52\end{array}$

5.1 Introduction . . . . . . . . . . . . . . 52

5.2 Anderson localized optical fiber . . . . . . . . . . . 56

5.3 Impact of the site size on localization radius . . . . . . . . . 60 
5.4 Impact of the fill-fraction on the localization radius . . . . . . . . 68

5.5 Impact of refractive index contrast . . . . . . . . . . . 70

5.6 Glass Anderson localized optical fibers . . . . . . . . . . 71

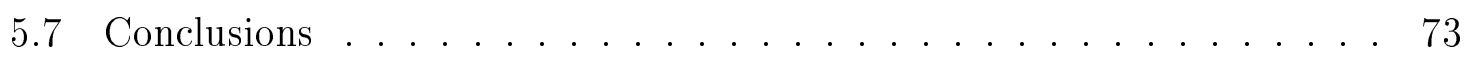

6 Transverse Anderson localization in a glass optical fiber with disor$\begin{array}{ll}\text { dered air voids } & 75\end{array}$

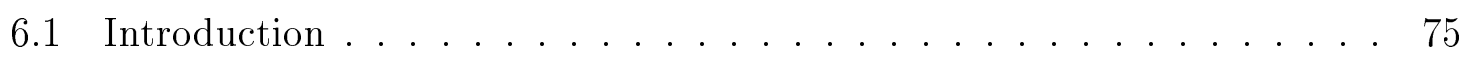

6.2 Disordered optical fiber with random air voids . . . . . . . . . 77

6.3 Localization near the outer boundary . . . . . . . . . . . 80

6.4 Non-uniform distribution of disorder . . . . . . . . . . . 83

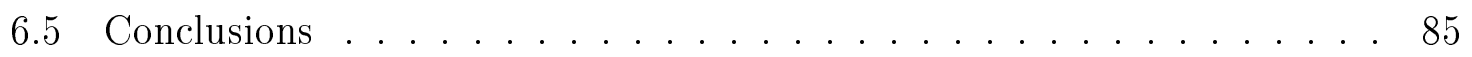

7 Multi-beam propagation in Anderson localized optical fibers $\quad 87$

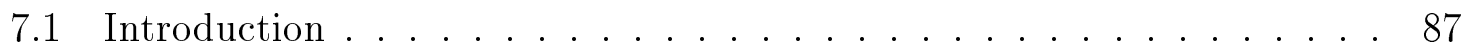

7.2 Multiple-beam propagation through a disordered fiber . . . . . . . . . 90

7.3 Impact of macro-bending on the drift of the center of localized beam - 95

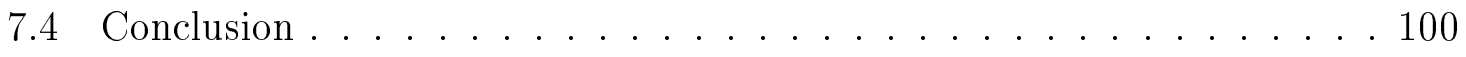

8 Image transport through the disordered polymer optical fiber mediated by transverse Anderson localization $\quad 101$

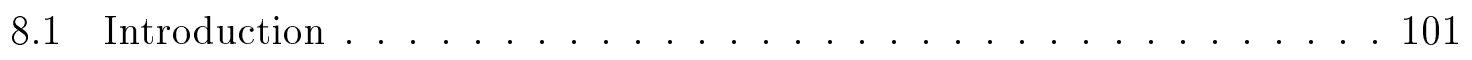

8.2 Image transport in the disordered fiber . . . . . . . . . . 105 
8.3 Image quality assessment metrics . . . . . . . . . . . . . 106

8.4 Comparison with commercial multicore image fibers . . . . . . . . . 108

8.5 Sources of systematic image degradation $\ldots \ldots \ldots \ldots 111$

8.6 Disorder-induced localization is responsible for enhanced image transport112

8.7 Disorder improves image transport quality . . . . . . . . . . . . 115

8.8 Image quality versus propagation distance $\ldots \ldots \ldots \ldots$

8.9 Impact of the wavelength on image quality . . . . . . . . . 118

8.10 Impact of the disorder refractive index difference on image quality . . . 119

8.11 Signal attenuation along the fiber $\ldots \ldots \ldots \ldots$

8.12 Comparison with other advanced fiber-based imaging methods . . . . 121

8.13 Conclusion . . . . . . . . . . . . . . . . . . . . . 123

A.1 FD-BPM code in C language . . . . . . . . . . . . . . . . 135

A.2 FD-BPM code in Matlab . . . . . . . . . . . . . . . . 148

B Shell scripting for compiling and submitting the jobs

B.1 Building a job on Peregrine $\ldots \ldots \ldots \ldots \ldots \ldots \ldots$

B.2 Submitting a job of Peregrine $\ldots \ldots \ldots \ldots \ldots \ldots$ 
B.3 Compiling multiple jobs on AVI . . . . . . . . . . 156

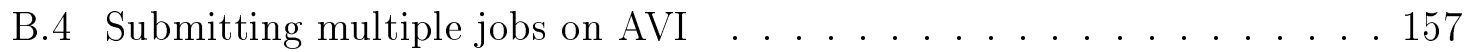

C Stability analysis for $2^{\text {nd }}$ order Runge-Kutta method 


\section{List of Figures}

1.1 a) Alumina spheres surrounded by Styrofoam inside the metallic tube. (b) Titanium dioxide particles (c) Gallium arsenide powder and (d) network of aluminum beads. The image is from Ref. [6]. . . . . . . . . 3

1.2 (a) The schematic of the photonic crystal with no disorder in which the beam expands as it propagates versus the case with disorder in which the beam expansion is smaller after the same propagation distance. The intensity profile at the end of the periodic lattice with (b) no disorder,

(c) moderate disorder, and (d) large disorder. Images are from Ref. [16].

1.3 (a) The schematic of the one dimensional AlGaAs waveguides. The intensity profile at the end of the sample for (b) no disorder, (c) moderate disorder, and (d) high disorder. The image is from Ref. [17]. . . . . . . 6

1.4 A concise history of Anderson localization (AL) and the contribution of this work to the field. ................ 7

2.1 Normalized power versus propagation distance in a dielectric waveguide with the metallic walls. In the numerical calculations $d x=d y=0.3 \lambda$ and $d z$ is calculated using the stability criterion. . . . . . . . . 21 
2.2 The field absorption by the lateral TBC boundaries as the wave propagates in a waveguide with the $2 \mathrm{D}$ profile. . . . . . . . . . 24

2.3 The normalized amplitude vs propagation distance for a Gaussian beam calculated using FD-BPM and the analytical formula. . . . . . . . 25

2.4 The field amplitudes calculated using FD-BPM and COMSOL for propagation distances of (a) $20 \mu \mathrm{m}$, (b) $300 \mu \mathrm{m}$ and (c) $400 \mu \mathrm{m} . \ldots 26$

3.1 SEM image of the polished polymer disordered fiber. . . . . . . . . . 29

3.2 Zoomed-in SEM image of the refractive index profile of the disordered polymer fiber. The PMMA sites are in dark color while PS sites are light gray. . . . . . . . . . . . . . . . . 30

3.3 Original Strands of PMMA and PS. . . . . . . . . . . . 32

3.4 Randomly mixed strands of PMMA and PS inside the square preform. 33

3.5 (a) Scanning electron microscope, (b) Sputterer. . . . . . . . . 34

3.6 The coupling and detection sections of the experimental setup. . . . . . 37

3.7 (a)-(e) Intensity profiles for five different locations of the input beam. . 38

4.1 a) A sample refractive index profile that we have used for our simulations $(p=50 \%)$; the black regions have refractive index $n_{1}=1.49$, while the white regions have refractive index $n_{2}=1.59$, b) SEM image of a polished fiber tip: the high and low refractive index regions are not distinguishable in this SEM image, and c) SEM image of a fiber tip exposed to the solvent, where the feature sizes in (c) are substantially smaller than the localized beam diameter, as shown in Fig. 4.3. . . . . 44 
4.2 a) Near-field intensity profile after $60 \mathrm{~cm}$ of propagation from experiment, b) Near-field intensity profile after nearly $5 \mathrm{~cm}$ of propagation in a sample ALOF from b) experiment and c) simulation. For comparison, we note that the total side width of each figure is $250 \mu \mathrm{m} . \ldots . . . .46$

4.3 The region highlighted in green corresponds to one standard deviation in each direction around the average experimental measurement of the localization length parameter represented by $\xi_{\text {avg }} \pm \sigma_{\xi}$. The measurements are carried over fibers each with an approximate length of $5.5 \mathrm{~cm}$. The region highlighted in black corresponds to theoretical simulation of the effective beam radius $\xi_{\text {avg }} \pm \sigma_{\xi}$ as a function of propagation distance. 47

4.4 Cross section of the intensity profile of the localized beam averaged over 100 samples of raw data from simulations and 100 samples from experiments in $\mathrm{dB}$ units. . . . . . . . . . . . . . . 48

4.5 The 3-dimensional plot of the wavelength-wavevector-effective index $(\lambda$ $\left.\mathrm{K}_{z}-\mathrm{n}_{\mathrm{eff}}\right)$ for 50 eigenmodes. . . . . . . . . . . . . . . 49

4.6 The intensity profiles of the localized modes for different values of $n_{\text {eff }}$ and at different regions at the tip of the fiber. The black circles indicate the PMMA sites with the refractive index of 1.49. The intensity profiles are zoomed in square regions with the side widths of $12 \mu \mathrm{m} . \ldots . . .50$ 
5.1 (a) An example cross section of a fiber with site size of $0.9 \mu m$ used for our simulations, corresponding to a fiber of side width equal to $250 \mu \mathrm{m}$. (b) and (c) SEM images of the fibers with the side width of $150 \mu \mathrm{m}$ and $250 \mu \mathrm{m}$, respectively. All three figures (a), (b), and (c) only feature a $24 \mu m \times 24 \mu m$ region of the total fiber cross section for a more clear view. The captions on the SEM images show $15 \mathrm{KV}$ at 5000x magnification, with a marker to show the physical scale of the images. The darker regions in the SEM images indicate the PMMA

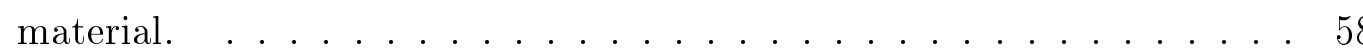

5.2 Effective beam radius versus propagation distance for different values of the site size: $d=0.9 \mu \mathrm{m}$ corresponding to a fiber side width of $250 \mu \mathrm{m}$, and $d=0.6 \mu \mathrm{m}$ corresponding to a fiber side width of $150 \mu \mathrm{m}$. The mean beam radius in the case of the fibers with side width of $150 \mu \mathrm{m}$ is greatly affected by the large refractive index step at the boundary of the fiber, otherwise it would have been even larger than that of the fibers with side width of $250 \mu \mathrm{m}$. We note that the one standard-deviation regions for experimental measurements marked with green and red color are from the measurements at the end of the fiber samples at the 5.5 cm length. . . . . . . . . . . . . . . . . . . 6 61 
5.3 (a) and (b) show the refractive index profile of a sample optical fiber with $1 \mathrm{~mm}$ side width taken at different locations along the fiber $(5$ $\mathrm{cm}$ apart). The images are taken with an optical microscope and are zoomed in at a small region on the cross section of the fiber and clearly show that the refractive index profile remains invariant along the fiber over the $5 \mathrm{~cm}$ long samples. Similarly, the (c) - (d) pair, (e) - (f) pair, and $(\mathrm{g})-(\mathrm{h})$ pair are taken each at $5 \mathrm{~cm}$ apart locations along the fibers where the optical microscope is zoomed in over the same regions for each pair but different regions for different pairs across the fiber tip.

5.4 The effect of reducing the incident wavelength $(\lambda)$ on the localization $\operatorname{radius}(\xi)$ versus propagation distance. . . . . . . . . . .

5.5 Cross section of the intensity profile of the localized beam averaged over 100 samples of raw data in dB units. (a) shows a comparison between the experimental results for fibers with side width of $150 \mu \mathrm{m}$ and $250 \mu \mathrm{m}$. (b) shows a comparison between the experimental and numerical results for fibers with side width of $150 \mu \mathrm{m}$, where the difference between simulation and experiment is caused by the larger variation in the experimental results (see Fig. 5.2), and also the noise in the CCD beam profiler at low intensities. . . . . . . . . . . 67

5.6 Effective beam radius versus propagation distance for different values of the fill-fraction of $p=40 \%$, and $p=50 \%$. The optimal fill-fraction of $p=50 \%$ results in the lowest effective beam radius and localization length. . . . . . . . . . . . . . . . . . 68 
5.7 Effective beam radius vs propagation distance for different values of refractive index contrast $\Delta n$. Larger index contrast results in smaller

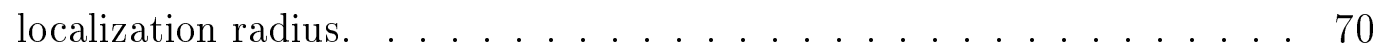

5.8 Effective beam radius vs propagation distance for different values of fill-fraction, $\mathrm{p}$, in glass disordered optical fibers with random air voids.

5.9 Exponential decay of the average intensity for different values of fillfractions, $p$, in glass disordered optical fibers with random air voids. . . 72

6.1 (a) SEM image of the glass optical fiber with random air voids. For ease of viewing, the polymer coating has been removed. (b) Refractive index profile used in our simulations. . . . . . . . . . . . . . . 79

6.2 The experimental measurement of the near-field intensity when the beam is launched near the center of the fiber, where no localization is observed. . . . . . . . . . . . . . . . . . . . . 79

6.3 Near-field intensity measurements at the output facet of the disordered fiber samples for 4 different launch positions. $\ldots \ldots \ldots$. . . . 80

6.4 Near-field intensity simulations at the output facet of the disordered fiber for 4 different launch positions. . . . . . . . . . . . 81 
6.5 (a) The region highlighted in red corresponds to one standard deviation in each direction around the average experimental measurement of the localization length parameter represented by $\xi_{\text {avg }} \pm \sigma_{\xi}$. The region highlighted in black corresponds to theoretical simulation of the effective beam radius $\xi_{\text {avg }} \pm \sigma_{\xi}$ as a function of propagation distance. (b) Cross section of the intensity profile of the highest peak in the localized beam averaged over 100 samples of raw data from simulations and 100 samples from experiments in $\mathrm{dB}$ units. . . . . . . . . . . . . 82

6.6 (a) Density plot shows the air fill-fraction over the tip of the fiber, where the disorder level is generally higher near the outer boundary than the central regions. (b) Segmentation of fiber to different regions for averaging over the angular coordinate, where red and blue colors are used in order to make it easier for the reader to distinguish the regions closer to the center versus regions closer to the outer boundary of the fiber. (c) Air fill-fraction averaged over the angular coordinate as a function of the radial coordinate over the tip of the fiber. The error bars signify the change in the value of the air fill-fraction, if the global image threshold varies by 0.07 around an Otsu's threshold of 0.37 . . 84

6.7 Simulation of the near-field intensity profile when the beam is launched near the center of the fiber, for uniform disorder distribution with (a) $3 \%$ air fill-fraction, (b) $6 \%$ air fill-fraction, and (c) $10 \%$ air fill-fraction. (d) Cross section of the intensity profile for uniformly disordered fibers with $3 \%, 6 \%$, and $10 \%$ air fill-fraction, where the beam is launched near the center of the fiber. All figures are plotted for the intensity profile

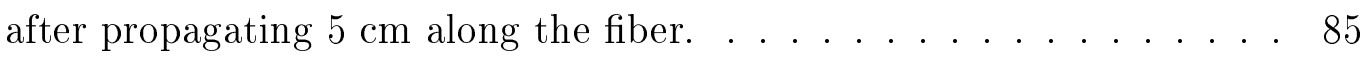


7.1 Multiple-beam propagation in a $5 \mathrm{~cm}$-long p-ALOF (a) simulation for five beams; (b) experiment for two beams; and (c) experiment for two beams with different wavelengths. All beams are at $405 \mathrm{~nm}$ wavelength, except the bottom-middle beam in subfigure (c), which is at $633 \mathrm{~nm}$

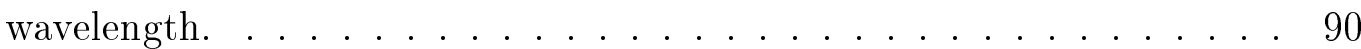

7.2 (a) The cross-section of the intensity profiles of the localized beam at $405 \mathrm{~nm}$ wavelength for 20 different realizations of the p-ALOF randomness are shown using numerical simulations, where the profiles are plotted on top of each other to capture the expected variations. (b) The experimental measurements of the beam width are shown in a histogram from 92 separate measurements. . . . . . . . . . . . . . . 91

7.3 (a) Similar to Fig. 7.1(a), but the beam intensity is averaged over 20 different realizations of randomness. Substantial beam clean-up is observed compared with Fig. 7.1(a) due to the averaging. (b) Crosssection of the intensity profile where the results of 20 different realizations are plotted on top of each other to show the extent to which the beams overlap due to the statistical nature of the problem. (c) Same as (b) but the cross-sectional intensity is plotted for the average of the 20 different realizations. All figures are shown at $405 \mathrm{~nm}$ wavelength. . 93 
7.4 (a) Original index profile of the p-ALOF. (b) Conformally modified refractive index profile of a $\mathrm{p}$-ALOF with bend radius of $0.5 \mathrm{~mm}$. (c) Effective refractive index difference between the low-index and highindex sites for different values of bend radius as a function of the location across the fiber profile. The fiber is assumed to be bent in the $\mathrm{x}$ direction. The dimensions of subfigures (a) and (b) are $300 \mu m$ on each

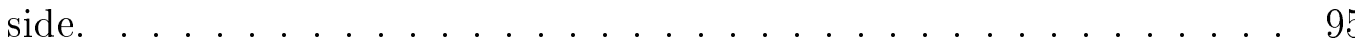

7.5 Trajectory of the beam center across the fiber as the beam propagates along a $5 \mathrm{~cm}$ segment for different bend radii in a) polymer fiber at $\lambda=405 \mathrm{~nm}, \mathrm{~b})$ polymer fiber at $\lambda=633 \mathrm{~nm}, \mathrm{c}$ ) glass fiber at $\lambda=633 \mathrm{~nm} .96$

7.6 Experimental measurement of the intensity of the propagated light in a fiber with (a) no bend, (b) bend radius of $1 \mathrm{~mm}$. The wavelength is $405 \mathrm{~nm}$ and the fiber sample is $15 \mathrm{~cm}$ long. No shift is observed, which is also consistent with the simulations in Fig. 7.5(a). We have intentionally saturated the CCD camera slightly to illustrate the location of the beams with respect to the boundary of the fiber for easier comparison. 97

7.7 Histogram of the experimental measurements of the beam width from 72 separate measurements in bent fibers with the bend radius of approximately $1 \mathrm{~mm}$. The localization behavior holds for the majority of the 72 random realizations explored in this figure. . . . . . . . . 98 
7.8 Beam intensity of the propagated light after $5 \mathrm{~cm}$ of propagation in a bent p-ALOF with $R=0.5 \mathrm{~mm}$, when the light is launched closer to the (a) inside of the bend, (b) center of the fiber, and (c) outside of the bend. (d) Cross section of the beam intensity averaged over 20 samples for the beam in subfigure (a) in red versus the beam in subfigure (b) in green color versus the beam in subfigure (c) in blue color. . . . . . . . . 99

8.1 Magnified SEM image of a portion of the tip of the (a) p-ALOF and (b) glass disordered fiber. For p-ALOF in (a), the PMMA (PS) sites are darker (lighter) in color. For the glass disordered fiber, the darker sites are the air voids. The $4 \mu \mathrm{m}$ scale-bar applies to both images. . . . 104

8.2 Elements of a group on 1951 U.S. Air Force test target (1951-AFTT). . 105

8.3 Transported images of different numbers through $5 \mathrm{~cm}$ of $\mathrm{p}$-ALOF, (a)-(d), related to the group 3 on the 1951-AFTT (experimental measurements). . . . . . . . . . . . . 106

8.4 Transported images of the numbers through $5 \mathrm{~cm}$ of p-ALOF, (a)-(d), related to the group 4 on the 1951-AFTT (experimental measurements). 106

8.5 Transported images related to group 5 of the 1951-AFTT test chart in (a) p-ALOF, (b) FIGH-10-350S image fiber and (c) FIGH-10-500N image fiber (experimental measurements). The scale bar in (b) is $30 \mu \mathrm{m}-$ long and the same scale bar can be used for (a) and (c). Each fiber is approximately $5 \mathrm{~cm}$ long. The MSSIM image quality values for the images are: (a) 0.5877, (b) 0.5501, and (c) 0.5591. . . . . . . . 109 
8.6 Transported images related to group 5 of the 1951-AFTT test chart in (a) p-ALOF, (b) FIGH-10-350S image fiber and (c) FIGH-10-500N image fiber (numerical simulations). The scale bar in (a) is $30 \mu \mathrm{m}$-long and the same scale bar can be used for other subfigures. Each fiber is approximately $5 \mathrm{~cm}$ long. The top row (subfigures (a), (b), and (c)) can be compared with the experimental results shown in Fig. 8.5. Subfigures (d), (e), and (f) in the bottom row are the same as Subfigures (a), (b), and (c) in the top row, except the images are saturated by changing the color axis in gray-scale colormap in Matlab. The color axis of $[0,1]$ in subfigures (a), (b), and (c) is changed to color axis of $[0,0.3]$ in $(d)$, (e), and (f). The MSSIM image quality values for the images are: (a) 0.637, (b) 0.615, and (c) 0.6257. The MSSIM values for (d), (e), and (f) are the same as (a), (b), and (c), respectively. . . . . . . . 110

8.7 The intensity profile of the "UWM" image after $5 \mathrm{~cm}$ of propagation and at the wavelength of $405 \mathrm{~nm}$ in a (a) p-ALOF, (b) modified image fiber with the refractive index difference of 0.1 between the cores and the clad, using numerical simulations. The scale bar in (a) is $20 \mu \mathrm{m}$ long and the same scale bar can be used for (b). The MSSIM image quality value for the images are: (a) 0.8923 and (b) $0.6263 . \quad \ldots . . .113$

8.8 The MSSIM metric for image quality assessment is compared for periodic image fibers as a function of the core periodicity $\Lambda$. The blue squares represent disorder-free $(\Delta=0)$ periodic cores of radius $1.45 \mu \mathrm{m}$. The red circles and cyan diamonds represent disordered $(\Delta \neq 0)$ periodic cores of mean radius $1.45 \mu \mathrm{m}$ with uniform random radius variations in the interval $[-0.3 \mu \mathrm{m}, 0.3 \mu \mathrm{m}]$ and $[-0.9 \mu \mathrm{m}, 0.9 \mu \mathrm{m}]$, respec-

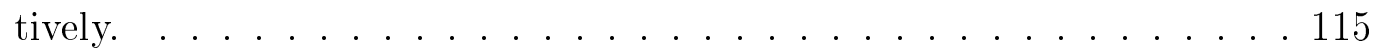


8.9 (a) The refractive index profile of a periodic structure with the cores radii of $1.45 \mu \mathrm{m}$ and the core to core distances of $3.9 \mu \mathrm{m}$. (b) The disordered structure with the variation of $\Delta=0.3 \mu \mathrm{m}$ in its cores radii. (c) The transported image after $5 \mathrm{~cm}$ of propagation in a periodic fiber with the refractive index profile in (a). (d) The transported image after $5 \mathrm{~cm}$ of propagation in a periodic fiber with the refractive index profile in (b). The scale bar in (d) is $65 \mu \mathrm{m}$ and the same scale bar can be used for $(\mathrm{a})-(\mathrm{c}) . \ldots \ldots \ldots \ldots \ldots \ldots$

8.10 MSSIM is plotted as a function of propagation distance for the disorderfree $(\Delta=0)$ periodic multicore image fiber in blue squares, the disordered case of $\Delta=0.3$ in red circles, and the disordered polymer Anderson localized fiber p-ALOF in cyan diamonds. . . . . . . . . 117

8.11 Numerical simulation of transported images related to group 5 of the 1951-AFTT test chart in (a) FIGH-10-350S image fiber, (b) FIGH-10$500 \mathrm{~N}$ image fiber, and (c) p-ALOF. Each fiber is approximately $5 \mathrm{~cm}$ long. The simulation wavelength is $633 \mathrm{~nm}$ and lower quality imaging is obtained, as expected, when compared with Fig. 8.6 at $405 \mathrm{~nm}$ wavelength. (d) is similar to (c), except an air-glass material with the index difference of 0.5 is assumed instead of 0.1 related to the polymer p-ALOF of (c). The scale bar in (d) is $20 \mu \mathrm{m}$ long and the same scale bar can be used for (a), (b), and (c). The MSSIM image quality value for the images are: (a) 0.3385, (b) 0.5396, (c) 0.6119, and (d) 0.6509. . 119 
8.12 Transported images of the numbers " 1 " and "6" from group 3 of the 1951AFTT test chart through a $16 \mathrm{~cm}$-long p-ALOF sample are shown using a white light source (experimental measurements). The scale bar in (a) is $120 \mu \mathrm{m}$ long and the same scale bar can be used for (b). . . . . . . 121 


\section{ACKNOWLEDGMENT}

I would like to express my gratitude towards many individuals whose help and assistance made it possible for me to accomplish this work. First, I deeply thank Professor Arash Mafi, who gave me the opportunity to work on this project. Without his perpetual mentorship, patience, support and invaluable inspiration, I would have not been able to establish the comprehension and understanding of my research. I would like to thank my committee members Professors George Hanson, Chiu Law, Hossein Hosseini and Rani El-Hajjar for their time and valuable suggestions. I would like to thank Craig R. Mirr and Ryan J. Frazier for their assistance in performing the experimental measurements. I would like to thank Professor John Ballato and Thomas Hawkins from the Material Science department at Clemson University for providing the glass optical fiber with disordered air voids. I would like to thank Dr. Karl W. Koch from Corning Incorporated whose feedback and ideas helped me improve the quality of this work. I would like to thank David J. Welker from Paradigm Optics Inc. for providing the initial polymer strands and drawing of the polymer optical fiber. I would like to thank Dr. Krishna M. Gundu and Dr. Aramais Zakharian from whom I learned a lot about the immense world of computational science. I would like to thank Mehdi Mortazavi for the fruitful discussions on the mechanical properties of the polymers. I would like to thank Jason Bacon for assisting in using the high performance computing facilities (HPC) at UWM. I would like to thank Drs. Steven Hardcastle and Heather Owens for the SEM images. I would like to thank National Science Foundation for providing the financial support under grant number 1029547. I would like to thank Drs. Ad Lagendijk, Diederik S. Wiersma, Moti Segev, Yaov Lahini, Yaron Silberberg and Alexander Szameit for their pioneering works on transverse Anderson localization of light that help me understand the importance of working on this dissertation. I would like to thank all of my labmates Dr. Parisa Gandomkar Yarandi, 
Craig Mirr, Hamed Pourbeyram, Seyed Rasoul Hosseini, Dr. Elham Nazemosadat, Seyedmohammad (Arash) Mousavi, Ramak Vejdanpak, Aaron Mueller, Ryan Frazier and Dr. Rodrigo Acuna for making a positive atmosphere in our lab over the period of my stay at the Photonics research group. I would like to thank my friends Mojtaba Heydar, Ali Moghtaderi, Abdolreza Javadi, Mohammad Reza (Oveis) Shaeri, and Ebrahim Forati for all the good times that we had in Milwaukee. I would like to thank my girl friend Amber for all her support and understandings that helped me focus on my work far away from my family. Last but not least, I would like to thank my beloved parents, Nematollah and Ashraf, my brothers, Kamran and Javad, and my sister, Farnoosh, for their unreserved love and uplifting motivations while I was thousands of kilometers away from them. 


\section{Chapter 1}

\section{Introduction}

\subsection{Anderson localization}

Philip W. Anderson in his 1958 paper [1] conjectured that beyond a critical level of disorder in electronic systems, localized electronic states develop, diffusion process stops and a phase transition from metal to insulator happens. Despite the theoretical predictions, it is extremely difficult to observe Anderson localization in an electronic system because of the thermal lattice vibrations and the electron-electron scattering, [2]. On the other hand, Anderson localization is a wave phenomenon and can be observed in any wave system including optics [3-5]. In contrast to electrons, photons do not interact with each other and the thermal lattice vibrations are negligible for photons in room temperature. Multiple scattering in disordered optical systems results in the confined states that can trap the light. Since the theoretical prediction of localization in wave systems [3], there have been many efforts to observe Anderson localization experimentally. For any level of disorder in one-dimensional (1D) and two-dimensional (2D) disordered systems, all the states are localized as long as the dimensions of the 
disordered system are larger than the width of the localized states [6]. Observation of Anderson localization in three-dimensional (3D) systems is challenging because of the requirement for strong scattering. This is the reason behind the diffusive scattering of light in day to day phenomena of light scattering by disordered media such as clouds, white marbles and biological tissues. In order to determine Anderson localization of waves in the disordered systems, the wave transmission can be investigated. In the diffusive regime, the transmission decays linearly with the length of the structure as opposed to the exponential decay for Anderson localization regime. In the experimental observations of Anderson localization, the difficulty is in distinguishing the trace of Anderson localization from the exponential decay triggered by loss. The experiments for the observation of Anderson localization need to be performed in a regime in which the material loss is minimal. The impact of loss is not detrimental for the observation of Anderson localization in disordered structures, yet the localization might seem stronger due to the exponential decay caused by loss.

In order to observe Anderson localization in any system, the Ioffe-Regel condition [7] needs to be satisfied that is $k l \leq 1 . k$ is the wave vector and $l$ is the scattering mean free path. According to this condition, Anderson localization can occur in a system only if the scattering is strong enough. The smaller scattering mean free path, $l$, in the optical system can be provided by stronger scattering or larger difference between the refractive indices of the disordered sites. It should also be noted that the scattering mean free path of photons is a dispersive phenomenon. A higher energy photon (shorter wavelength) results in a stronger scattering and a smaller scattering mean free path. On the other hand, for a long wavelength the mean free path becomes large because of weak scattering.

Anderson localization has been experimentally observed in 1D, 2D and 3D disordered structures and for different wave systems such as optics, microwave, and ultrasound. 


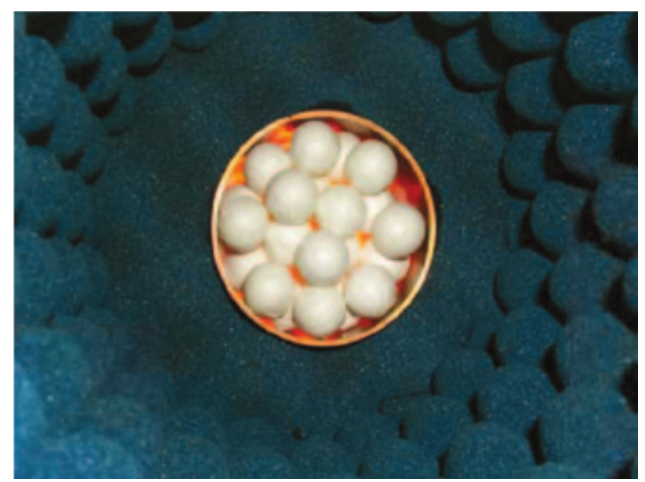

(a)

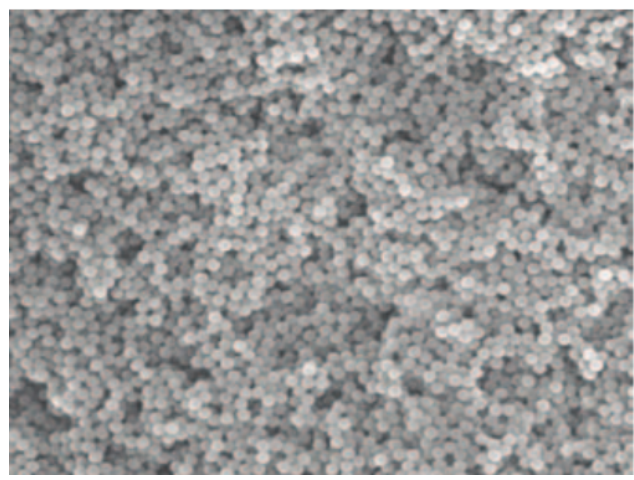

(c)

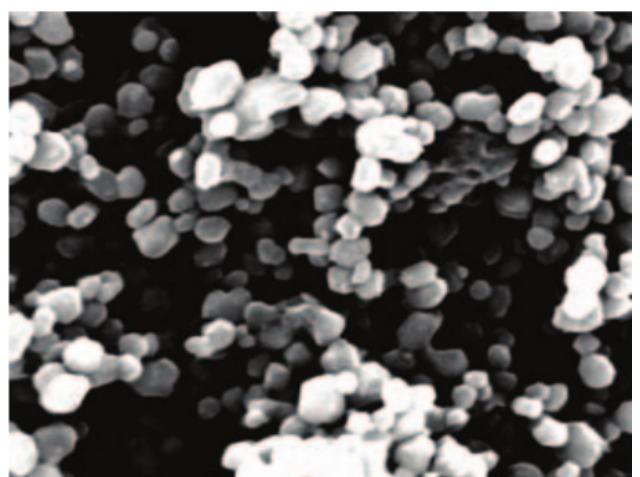

(b)

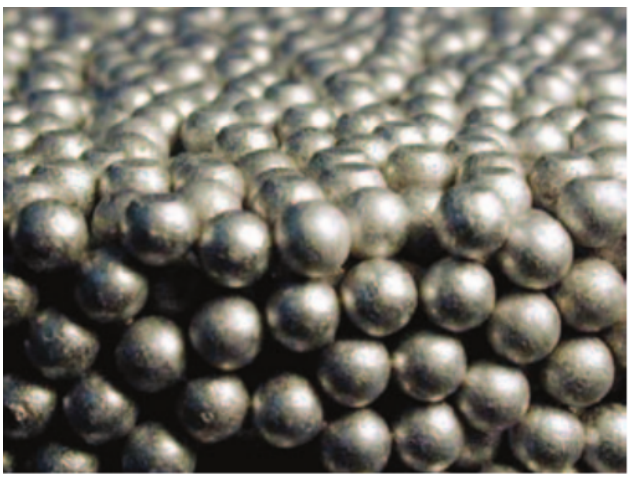

(d)

Figure 1.1: a) Alumina spheres surrounded by Styrofoam inside the metallic tube. (b) Titanium dioxide particles (c) Gallium arsenide powder and (d) network of aluminum beads. The image is from Ref. [6].

The first pioneering experiments for Anderson localization in the microwave regime were carried out in a quasi 1D system of alumina spheres (permittivity of 9.8) inside a metallic tube shown in Fig. 1.1(a), [8], and in a 2D structure of disordered rods (permittivity of 9) [9].

In the optical regime, the anomalous diffusion was first observed in a sub-micron titanium power (refractive index of 2.7), Fig. 1.1(b) [10]. In order to minimize the impact of loss, GaAs powder shown in Fig. 1.1(c) (refractive index of 3.5) at infrared wavelengths was used and clear traces of localization were observed [11].

Anderson localization of ultrasound was reported in a 3D network of aluminum beads [12] 
in which an ultrasound point source was used to excite the $3 \mathrm{D}$ system and traces of localization were observed in the transverse directions of the sample.

\subsection{Transverse Anderson localization}

According to the Ioffe-Regel criterion, aside from the scattering mean free path, $l$, a smaller wave vector, $k$, can also satisfy the localization condition. The idea of transverse Anderson localization was proposed in Refs. [13, 14]. In transverse Anderson localization, the disorder is only introduced in the transverse directions of a waveguide while it is invariant along the direction of propagation. The authors in Ref. [13] numerically showed that an initial beam of light in a transversely disordered waveguide can get localized in the transverse directions while propagating along the waveguide. The small transverse wave vector, $k_{T}$, compared with the propagating wave vector $k_{z}$, satisfies the Ioffe-Regel condition more easily for practical optical materials with lower refractive indices than the ones in the aforementioned experiments of wave localization.

In 2007, for the first time, transverse Anderson localization was experimentally observed in a photo-refractive crystal in which the disordered fluctuations with a refractive index contrast of $4 \times 10^{-4}$ were induced on top of a periodic lattice [15]. A schematic of the photo-refractive crystal is shown Fig. 1.2(a) [15]. The small refractive index contrast in such a system results in a large localized beam radius and its large variations for different realizations of randomness. The ensemble averaged intensity profiles after $1 \mathrm{~cm}$ of propagation in the photo-refractive crystal with no disorder, moderate and high levels of disorder are shown in Fig. 1.2 (b)-(d) [16]. The wave localization was concluded from the exponential decay of the tails of the ensemble averaged intensity profile. 

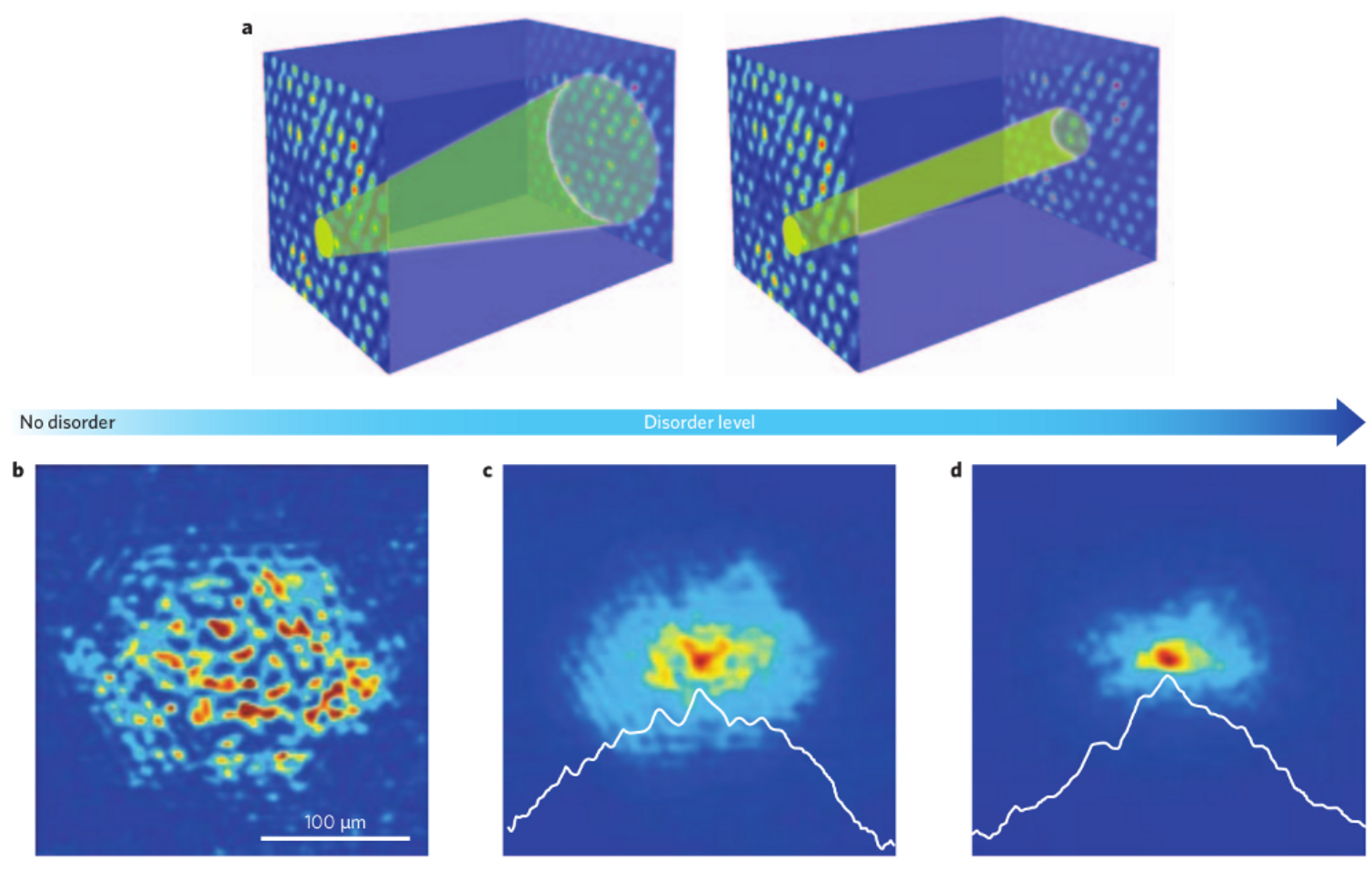

Figure 1.2: (a) The schematic of the photonic crystal with no disorder in which the beam expands as it propagates versus the case with disorder in which the beam expansion is smaller after the same propagation distance. The intensity profile at the end of the periodic lattice with (b) no disorder, (c) moderate disorder, and (d) large disorder. Images are from Ref. [16].

In the proposed method in Ref. [15], the disorder was introduced in the photo-refractive crystal using an interference pattern that limits its practical application.

Transverse Anderson localization was also observed in a 1D disordered lattice on an AlGaAs substrate [17]. A schematic of the 1D lattice from the Ref. [17] is shown in Fig. 1.3. The input beam of light was launched into one or a few waveguides. The initial beam may couple to the neighboring waveguides as it propagates. The intensity profiles at the end of periodic and disordered lattices are shown in Fig. 1.3(b)-(d). The authors showed that introducing disorder by randomly changing the widths of the waveguides results in the light localization on a few of the waveguides compared to the periodic case, in which the propagating initial beam of light couples to many waveguides in the lattice. 
(a)

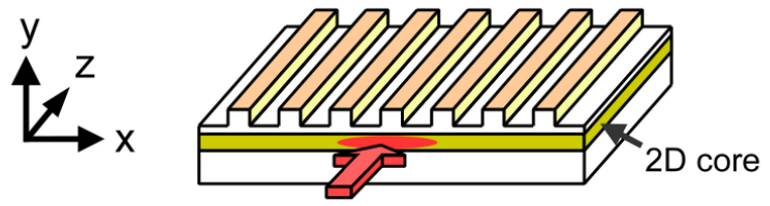

(b)

(c)

(d)

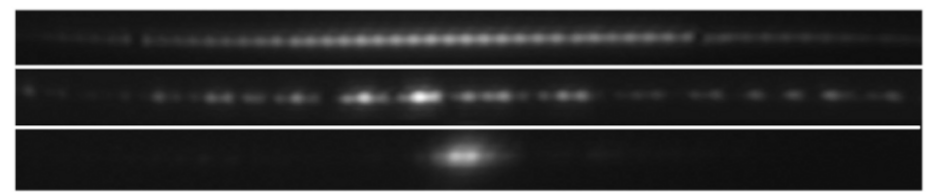

Figure 1.3: (a) The schematic of the one dimensional AlGaAs waveguides. The intensity profile at the end of the sample for (b) no disorder, (c) moderate disorder, and (d) high disorder. The image is from Ref. [17].

\subsection{Motivation for this work and outline of disserta- tion}

In order to use transverse Anderson localization in real world applications, the disorder should be implemented in a robust platform such as optical fiber. In here, the disorder is introduced in the transverse profile of an optical fiber for the effective light transport using transverse Anderson localization. The refractive index profile of the disordered fiber is composed of two materials with the refractive indices of $n_{1}$ and $n_{2}$ that are randomly distributed in the transverse profile of the fiber. The refractive index profile is invariant along the direction of propagation for all the samples that are investigated in this work. The disordered optical fibers provide the opportunities for further study of transverse Anderson localization and potential applications of Anderson localization such as image transport. In a diagram in Fig. 1.4, the contribution of this work to the field of transverse Anderson localization is compared with the previous works. In continue, the structure of the dissertation is outlined.

In Chapter 1, the numerical method for modeling the wave propagation in the disor- 


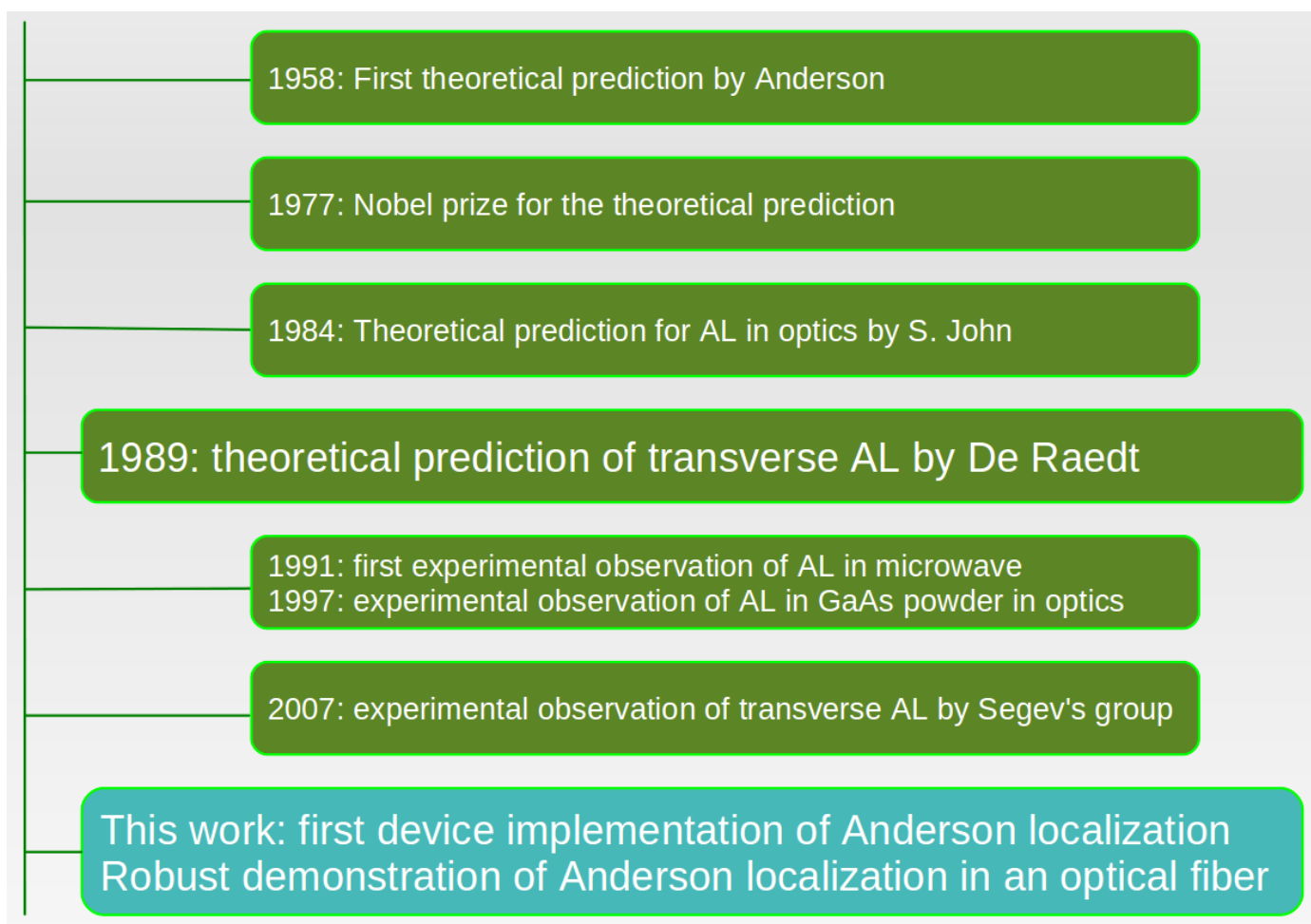

Figure 1.4: A concise history of Anderson localization (AL) and the contribution of this work to the field.

dered optical fibers is described. The beam propagation method is the standard approach for modeling the wave propagation in the optical fibers. In this work, the slowly varying envelope wave equation is solved using a $4^{\text {th }}$ order Runge-Kutta method [18]. The stability criterion for the numerical solution of the paraxial wave equation in a homogeneous medium is derived analytically. The derived stability condition is used as an initial condition for the stability of the numerical calculations in the inhomogeneous medium. The dissipation or symplecticity of the numerical method is discussed. The implementation of the transparent boundary conditions is described. The developed code is benchmarked with a commercial software and the analytical calculations. The samples of the developed codes in $\mathrm{C}$ language and Matlab are presented in Appendix A. The Shell scripts for compiling and running the $\mathrm{C}$ language code on the high performance computing facilities are presented in Appendix B. 
In Chapter 2, the procedures for fabrication and characterization of the disordered polymer optical fibers composed of PMMA and PS are described [19].

In Chapter 3, the experimentally measured localized beam radii in the disordered polymer fiber are compared with the numerical simulations. It is shown that the light propagation in the polymer disordered optical fiber results in a localized beam radius that is comparable to the ones in the conventional optical fibers. The transversely localized wave in the disordered optical fiber can propagate for long distances. The Anderson localization is identified by the exponential decay of the tails of the ensemble averaged intensity profile. The procedure for generating the refractive index profile in the simulations is illustrated [20]. The fully vectorial modes of the polymer disordered optical fiber are calculated using the MIT photonic bandgap (MPB) code [21].

In Chapter 4, the impacts of the design parameters on the light localization in the disordered optical fibers are investigated. The wave propagation in a glass optical fiber with disordered air voids and a fill-fraction of $50 \%$ results in a small localized beam radius. Additionally, the variation of the localized beam radius for different realizations of randomness is very small [22].

In Chapter 5, the wave localization in a glass optical fiber with nonuniform distribution of air voids is studied. The refractive index profile of the disordered glass fiber is directly used in the numerical simulations. The wave localization occurs in the regions with high fill-fraction of the air voids [23].

In Chapter 6, the simultaneous propagation of multiple beams in the disordered polymer optical fibers is examined. The impact of macro-bending on drifting the center of the beam is illustrated. For a strongly localized system, the drift of the beam center is negligible even for small bend radii [24].

In Chapter 7, the polymer Anderson localized optical fiber is used for high quality im- 
age transport. The theoretical calculations and the experimental measurements show that the quality of the transported images in the disordered polymer fiber is comparable or better than the ones in the commercially available imaging fibers. The structural similarity (SSIM) index is used for objective evaluation of the image quality. The role of transverse Anderson localization in improving the quality of the transported images is studied [25]. 


\section{Chapter 2}

\section{Finite Difference Beam Propagation}

\section{Method}

\section{$2.1 \quad$ Beam propagation method}

The beam propagation method (BPM) is one of the most popular techniques for modeling and simulation of light propagation in the optical waveguides [26]. In BPM, instead of wave equation, the slowly varying envelope approximation equation is solved that results in the reduce of the computational burden. In this work, the BPM is used to model the wave propagation in the disordered optical fibers. The wave equation is derived from the Maxwell's equations

$$
\left\{\begin{array}{l}
\nabla \times E=-j \omega \mu H \\
\nabla \times H=j \omega \epsilon E \\
\nabla \cdot\left(n^{2} E\right)=0 \\
\nabla \cdot H=0
\end{array}\right.
$$


as,

$$
\nabla \times \nabla \times E=n^{2} k^{2} E
$$

using $\nabla \times \nabla=\nabla(\nabla)-.\nabla^{2}$,

$$
\nabla(\nabla . E)-\nabla^{2} E=n^{2} k^{2} E
$$

where the electric field can be represented as $E(\mathbf{r})=\operatorname{Re}\left[A(\mathbf{r}) \exp \left(j n_{0} k_{0} z\right)\right]$. For the case of slow varying envelope

$$
\left|2 k n_{0} \frac{\partial A}{\partial z}\right| \gg\left|\frac{\partial A^{2}}{\partial z^{2}}\right|
$$

the wave equation can be approximated to

$$
\frac{\partial A}{\partial z}=\frac{1}{2 j k_{0}}\left[\frac{\partial^{2}}{\partial x^{2}}+\frac{\partial^{2}}{\partial y^{2}}+\left(k^{2}-k_{0}^{2}\right)\right] A
$$

In the disordered optical fibers, $k$ is a function of the transverse directions, $k(x, y)=$ $n(x, y) \omega / c . \quad k_{0}=n_{0} \omega / c$ where $n_{0}$ is the effective index of the propagating beam. For the disordered optical fiber, $n_{0}$ is calculated by weighted averaging of the refractive indices of the disordered sites. There are various numerical methods such as finite element, finite difference and fast Fourier transform for solving the slowly varying envelope equation, Eq. 2.5. In this work, the slowly varying envelope equation is discretized in the transverse direction using the finite difference method. An explicit Runge-Kutta method is used for advancing the field in the direction of propagation [18]. 


\subsection{Runge-Kutta method for solving the first order differential equation}

For an initial value problem

$$
y^{\prime}=f(x, y), \quad y\left(x_{0}\right)=y_{0}
$$

the $4^{\text {th }}$ order explicit Runge-Kutta method can be outlined as

$$
y_{n+1}=y_{n}+\frac{\Delta y}{6}\left(K_{1}+2 K_{2}+2 K_{3}+K_{4}\right)
$$

where

$$
\begin{aligned}
& K_{1}=f\left(x_{n}, y_{n}\right) \\
& K_{2}=f\left(x_{n}+0.5 \Delta y, y_{n}+0.5 \Delta y K_{1}\right) \\
& K_{3}=f\left(x_{n}+0.5 \Delta y, y_{n}+0.5 \Delta y K_{2}\right) \\
& K_{4}=f\left(x_{n}+\Delta y, y_{n}+\Delta y K_{3}\right)
\end{aligned}
$$

In order to implement the Runge-Kutta method, the right hand side of the Eq. 2.5 is discretized using the central difference method, then Runge-Kutta method is applied for updating the field values in the direction of propagation. In all the numerical algorithms, the stability and energy conservation are two important properties that guarantee the underlying physics of the problem. The stability and dissipation of the Runge-Kutta method will be discussed in the next sections. 


\subsection{Stability of the Runge-Kutta method}

In order to describe the stability issue in the numerical methods, first the stability analysis for solving the one-dimensional paraxial wave equation using the forward Euler method is carried out.

\subsubsection{Stability of forward Euler method}

The simplest numerical method for solving an ordinary differential equation is the Euler method. To solve the slow varying envelope equation, the forward Euler method and central difference method are used to discretize the equation in the ' $\mathrm{z}$ ' and ' $\mathrm{x}$ ' directions, respectively

$$
\frac{A_{i}^{m+1}-A_{i}^{m}}{\Delta z}=\frac{-j}{2 n_{0} k_{0}}\left[\frac{A_{i+1}^{m}-2 A_{i}^{m}+A_{i-1}^{m}}{\Delta x^{2}}+\left(k^{2}-k_{0}^{2}\right) A_{i}^{m}\right]
$$

where $A(i \Delta x, m \Delta z)=A_{i}^{m}$. To identify the stability condition for the discretized equation, Eq. 2.9, the von Neumann stability analysis is utilized [27]. If the electric field $A$ can be expanded in a finite Fourier series with $M$ harmonics,

$$
A=\sum_{m=1}^{M} A^{m} e^{j t i \Delta x}
$$

according to von Neumann stability analysis, for any wave vector ' $t$ ', there is a region, $\left|\frac{A^{m+1}}{A^{m}}\right|<1$, in which the numerical method is stable. In the following derivations only one term of the Fourier expansion is used because the behavior of each term of the series is the same as series itself. Using the definition of Eq. 2.10 in the discretized 
equation Eq. 2.9,

$$
A^{m+1}-A^{m}=\frac{-j \Delta z}{2 n_{0} k_{0}}\left[A^{m} \frac{e^{j t \Delta x}-2+e^{-j t \Delta x}}{\Delta x^{2}}+\left(k^{2}-k_{0}^{2}\right) A^{m}\right]
$$

then

$$
A^{m+1}=A^{m}\left[1+C\left(k^{2}-k_{0}^{2}\right)+C \frac{2 \cos (t \Delta x)-2}{\Delta x^{2}}\right]
$$

where

$$
C=\frac{-j \Delta z}{2 n_{0} k_{0}}
$$

using the identity

$$
\cos (x)=1-2 \sin ^{2}\left(\frac{x}{2}\right)
$$

the gain factor is

$$
\frac{A^{m+1}}{A^{m}}=\left[1+C\left(k^{2}-k_{0}^{2}\right)-4 C \frac{\sin ^{2}(t \Delta x / 2)}{\Delta x^{2}}\right]
$$

Since $C$ is pure imaginary,

$$
\left|\frac{A^{m+1}}{A^{m}}\right|=\sqrt{1+|C|^{2}\left(\left(k^{2}-k_{0}^{2}\right)-4 \frac{\sin ^{2}(t \Delta x / 2)}{\Delta x^{2}}\right)^{2}}>1
$$

As 2.14 shows, updating the field values using the Euler method results in an unconditionally unstable solution. In the next section, the stability criterion for the $4^{\text {th }}$ order explicit Runge-Kutta method is derived. 


\subsubsection{Stability of $4^{\text {th }}$ order Runge-Kutta method}

To simplify the derivation of the stability criterion for the Runge-Kutta method, the operator $H$ is defined as

$$
H=\frac{1}{2 k}\left[\frac{\partial^{2}}{\partial x^{2}}+\left(k^{2}-k_{0}^{2}\right)\right]
$$

so the slowly varying envelope equation can be written as

$$
\frac{\partial A}{\partial z}=-j H A
$$

If $\Delta z$ is the step size in the direction of propagation, $K_{1}, K_{2}, K_{3}, K_{4}$ in the $4^{\text {th }}$ order Runge-Kutta method can be written as

$$
\begin{aligned}
& K_{1}=-j H \Delta z A^{m} \\
& K_{2}=-j H \Delta z\left(A^{m}-j 0.5 H A^{m} \Delta z\right) \\
& \text { or } \\
& K_{2}=-j H \Delta z(1-j 0.5 H \Delta z) A^{m} \\
& K_{3}=-j H \Delta z\left(A^{m}-j H \Delta z(0.5-j 0.25 H \Delta z) A^{m}\right) \\
& \text { or } \\
& K_{3}=-j H \Delta z\left(1-0.5 j H \Delta z-0.25 H^{2} \Delta z^{2}\right) A^{m} \\
& K_{4}=-j H \Delta z\left(A^{m}+-j H \Delta z\left(1-0.5 j H \Delta z-0.25 H^{2} \Delta z^{2}\right) A^{m}\right) \\
& \text { or } \\
& K_{4}=-j H \Delta z\left(1-j H \Delta z-0.5 H^{2} \Delta z^{2}+0.25 j H^{3} \Delta z^{3}\right) A^{m}
\end{aligned}
$$


so the updated electric field is

$$
A^{m+1}=A^{m}+\frac{K_{1}+2 K_{2}+2 K_{3}+K_{4}}{6}
$$

substituting Eqs. 2.17 in Eq. 2.18 results in:

$$
\begin{aligned}
& A^{m+1}=A^{m}\left(1-\frac{j H \Delta z}{6}\left(6-j 3 H \Delta z-H^{2} \Delta z^{2}+0.25 j H^{3} \Delta z^{3}\right)\right) \\
& A^{m+1}=A^{m}\left(1-\frac{j H \Delta z}{6}\left(6-H^{2} \Delta z^{2}-j\left(3 H \Delta z-0.25 H^{3} \Delta z^{3}\right)\right)\right) \\
& \text { or } \\
& \frac{A^{m+1}}{A^{m}}=1-\frac{H \Delta z}{6}\left(3 H \Delta z-0.25 H^{3} \Delta z^{3}\right)-\frac{j H \Delta z}{6}\left(6-H^{2} \Delta z^{2}\right) \\
& \text { or } \\
& \frac{A^{m+1}}{A^{m}}=1-\frac{H^{2} \Delta z^{2}}{2}+\frac{H^{4} \Delta z^{4}}{24}-j\left(H \Delta z-\frac{H^{3} \Delta z^{3}}{6}\right)
\end{aligned}
$$

so

$$
\left|\frac{A^{m+1}}{A^{m}}\right|=\sqrt{\left(1-\frac{H^{2} \Delta z^{2}}{2}+\frac{H^{4} \Delta z^{4}}{24}\right)^{2}+\left(H \Delta z-\frac{H^{3} \Delta z^{3}}{6}\right)^{2}}
$$

To have a stable solution, $\left|\frac{A^{m+1}}{A^{m}}\right|<1$ :

$$
\begin{aligned}
& \left(1-\frac{H^{2} \Delta z^{2}}{2}+\frac{H^{4} \Delta z^{4}}{24}\right)^{2}+\left(H \Delta z-\frac{H^{3} \Delta z^{3}}{6}\right)^{2}<1 \\
& 1+\frac{H^{8} \Delta z^{8}}{24^{2}}-\frac{H^{6} \Delta z^{6}}{72}<1 \\
& \frac{H^{8} \Delta z^{8}}{24^{2}}<\frac{H^{6} \Delta z^{6}}{72}
\end{aligned}
$$


since $H^{6} \Delta z^{6} \geq 0$, to satisfy 2.21 :

$$
\frac{H^{2} \Delta z^{2}}{24^{2}}<\frac{1}{72}
$$

or

$$
|H \Delta z|<\frac{24}{\sqrt{72}}
$$

Inequality 2.23 shows that there is a stability region for the explicit $4^{\text {th }}$ order RungeKutta method. To determine the stability region using the von Neumann stability analysis, the $H$ operator is simplified as

$$
H=\frac{1}{2 k}\left[\frac{-4 \sin ^{2}(t \Delta x / 2)}{\Delta x^{2}}+\left(k^{2}-k_{0}^{2}\right)\right]
$$

where ' $\mathrm{t}$ ' is an arbitrary wave vector.

For the case of homogeneous medium, $k=k_{0}$, the $H$ operator is

$$
H=\frac{1}{2 k}\left[\frac{-4 \sin ^{2}(t \Delta x / 2)}{\Delta x^{2}}\right]
$$

for the worst case scenario that $\sin ^{2}(t \Delta x / 2)=1$

$$
|H|=\frac{2}{k \Delta x^{2}}
$$

so the stability criterion is

$$
\frac{\Delta z}{k \Delta x^{2}}<\frac{12}{\sqrt{72}}=\sqrt{2}
$$

that could be written as

$$
\frac{\lambda \Delta z}{\Delta x^{2}}<\frac{24 \pi}{\sqrt{72}}
$$

The stability analysis for the $2^{\text {nd }}$ order Runge-Kutta method is also presented in 
Appendix C. 


\subsection{Stability condition for a two-dimensional waveg- uide}

The paraxial wave equation for a waveguide with the $2 \mathrm{D}$ profile is

$$
\frac{\partial A}{\partial z}=\frac{1}{2 j k_{0}}\left[\frac{\partial^{2}}{\partial x^{2}}+\frac{\partial^{2}}{\partial y^{2}}+\left(k^{2}-k_{0}^{2}\right)\right] A
$$

If $A(x, y, z)=A(i \Delta x, k \Delta y, m \Delta z)=A_{i, k}^{m}$, using the central difference method the right hand side of the Eq. 2.29 can be discretized as

$$
\frac{\partial A}{\partial z}=\frac{-j}{2 n_{0} k_{0}}\left[\frac{A_{i+1, k}^{m}-2 A_{i, k}^{m}+A_{i-1, k}^{m}}{\Delta x^{2}}+\frac{A_{i, k+1}^{m}-2 A_{i, k}^{m}+A_{i, k-1}^{m}}{\Delta y^{2}}+\left(k^{2}-k_{0}^{2}\right) A_{i, k}^{m}\right]
$$

Similar to the 1D case, the stability criterion is

$$
|H \Delta z|<2 \sqrt{2}
$$

yet for the worst case scenario

$$
|H|=\frac{2}{k}\left[\frac{1}{\Delta x^{2}}+\frac{1}{\Delta y^{2}}\right]
$$

so the stability criterion is

$$
\frac{\Delta z}{k}\left[\frac{1}{\Delta x^{2}}+\frac{1}{\Delta y^{2}}\right]<\sqrt{2}
$$

In case that $\Delta x=\Delta y$, the stability criterion can be simplified as 


$$
\frac{\Delta z}{k \Delta x^{2}}<\frac{\sqrt{2}}{2}
$$

It should be noted that the derived stability criterion in this section is for the wave propagation in a homogeneous medium. However, the stability region for the inhomogeneous medium can be found by a numerical experimentation and starting from the stability condition of the wave propagation in the homogeneous medium.

\subsection{Symplecticity and dissipation}

In dissipative or nonsymplectic numerical methods [28], the energy dissipation in the solution cannot be described by the underlying physics. This energy dissipation could be the case for the long time integration problems such as for the wave propagation in a long optical fiber. In order to make sure about the symplecticity of the developed algorithm based on $4^{\text {th }}$ order Runge-Kutta method, the power is measured along the propagation direction in a dielectric waveguide with metallic walls. Metallic walls prevent the leakage of energy from the sides and any loss in the system should be attributed to the dissipation in the numerical algorithm. The normalized power along the propagation direction is presented in Fig. 2.1 for $d x=d y=0.3 \lambda$ and a $d z$ calculated using the stability criterion. The constant power for long propagation distance shows that the simulation parameters $(\mathrm{dx}, \mathrm{dy}$ and $\mathrm{dz}$ ) can be chosen so that there is no dissipation in the system.

Similar to the stability region, it is possible to have the developed numerical algorithm in a non-dissipative regime. The only degree of freedom here is the transverse mesh sizes and should be smaller than the wavelength for a non-dissipative solution.

There are implicit methods that are unconditionally symplectic, however, the implicit 


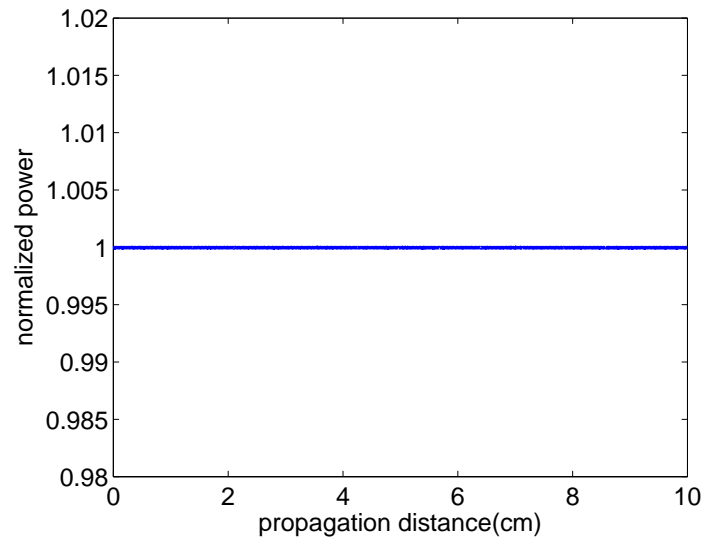

Figure 2.1: Normalized power versus propagation distance in a dielectric waveguide with the metallic walls. In the numerical calculations $d x=d y=0.3 \lambda$ and $d z$ is calculated using the stability criterion.

algorithms are more complex and need longer computational time than the explicit methods. 


\subsection{Transparent Boundary Condition for 1D waveg- uide}

In order to prevent the back reflections from the lateral boundaries of the simulation window in the beam propagation method, the transparent boundary conditions (TBCs) are implemented [29]. The concept of TBC simply is that the field values at the boundaries are equal to the field values at the previous step with some transverse phase shift because of the beam expansion in the transverse directions. For a onedimensional waveguide with the transverse profile in the ' $x$ ' direction, the transverse wave vector ' $k_{x}$ ' can be calculated using the field values at two steps away from the boundaries. The calculated phase shift is used as an approximation for the phase shift of the field values at the boundaries. Mathematically, if the electric field is defined as $A(i \Delta x, n \Delta z)=A_{i}^{n}, A_{i-1}^{n}$ and $A_{i}^{n}$ are the field values at the two steps away from the boundaries and:

$$
\frac{A_{i}^{n}}{A_{i-1}^{n}}=e^{-j \Delta x k_{x}}
$$

' $k_{x}$ ' is calculated using

$$
k_{x}=\frac{j}{\Delta x} \ln \frac{A_{i}^{n}}{A_{i-1}^{n}}
$$

so the updated field value at the boundary, $A_{i+1}^{n}$, is

$$
A_{i+1}^{n}=A_{i}^{n} e^{-j \Delta x k_{x}}
$$

where $k_{x}$ is calculated using the field values at the two previous steps. 


\subsection{TBC for a $2 \mathrm{D}$ waveguide}

The only difference in the implementation of TBCs for a $2 \mathrm{D}$ waveguide versus the $1 \mathrm{D}$ waveguide is the impact of phase direction. In the waveguides with the $2 \mathrm{D}$ profiles, an initial beam is expanding in both ' $\mathrm{x}$ ' and ' $\mathrm{y}$ ' directions simultaneously. In case that $\Delta x=\Delta y$

$$
\frac{A_{i-1, k-1}^{m}}{A_{i-2, k-2}^{m}}=e^{-j k_{r} \sqrt{2} \Delta x}
$$

then

$$
\frac{A_{i, k}^{m}}{A_{i-1, k-1}^{m}}=e^{-j k_{r} \sqrt{2} \Delta x}
$$

or

$$
A_{i, k}^{m}=A_{i-1, k-1}^{m} e^{-j k_{r} \sqrt{2} \Delta x}
$$

In order to show the effectiveness of the lateral absorbing boundaries, the contour plots of the field profiles of a propagating beam at different propagation distances are shown Fig. 2.2. 


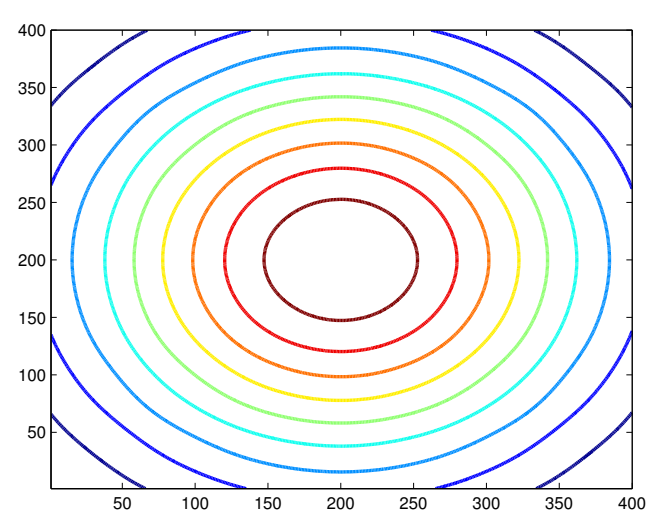

(a)

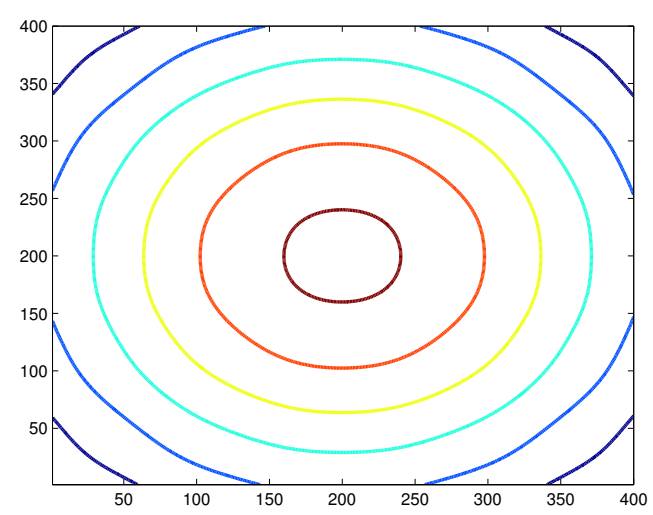

(b)

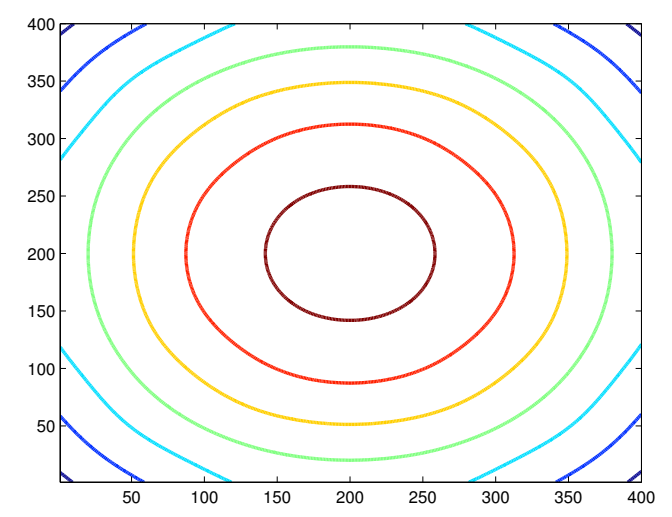

(c)

Figure 2.2: The field absorption by the lateral TBC boundaries as the wave propagates in a waveguide with the $2 \mathrm{D}$ profile.

It should be noted, that for most of the simulations of wave propagation in the disordered optical fibers, the tails of the localized beams are away from the boundaries of the simulation window and the TBCs are not required. 


\subsection{Benchmarking the developed numerical code}

In order to benchmark the developed code, first, the normalized amplitude of a Gaussian beam propagating in a homogeneous medium is calculated by the developed FDBPM code and compared with the values calculated using the analytical formula [30]:

$$
A p(z)=\frac{1}{\sqrt{1+\left(\frac{z}{z_{0}}\right)^{2}}}
$$

where the Rayleigh range $z_{0}=\pi W_{0}^{2} / \lambda$ and the initial beam waist $W_{0}$ is defined so that the initial Gaussian beam is $I(x, y, z=0)=\exp \left[-2 \frac{x^{2}+y^{2}}{W_{0}^{2}}\right]$. The normalized field amplitude versus propagation distance calculated using both methods are compared in Fig. 2.3.

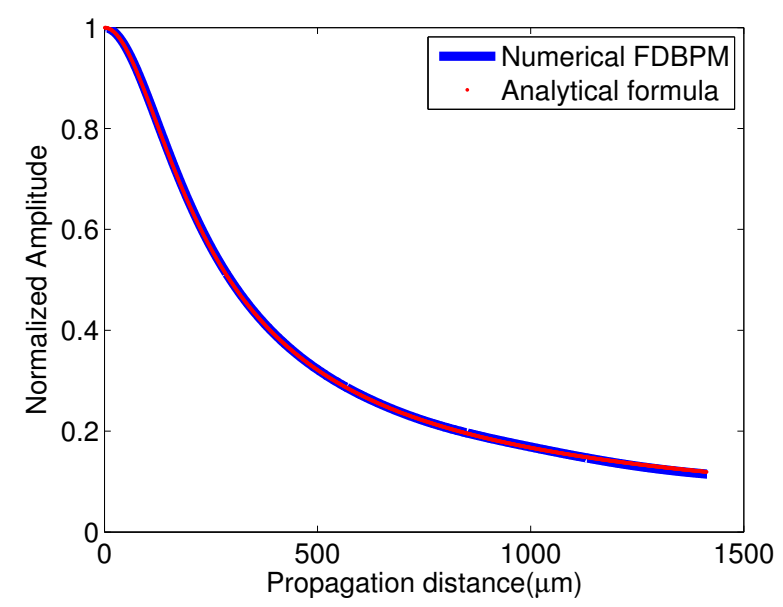

Figure 2.3: The normalized amplitude vs propagation distance for a Gaussian beam calculated using FD-BPM and the analytical formula.

The field profile of a propagating beam in a single mode 1D waveguide is calculated using the developed FD-BPM. In Fig. 2.4, the field profiles for different propagation distances are compared with ones calculated in COMSOL [31]. The absorbing bound- 
ary conditions in COMSOL are perfectly matched layers (PML) as opposed to the TBCs in the FD-BPM method.

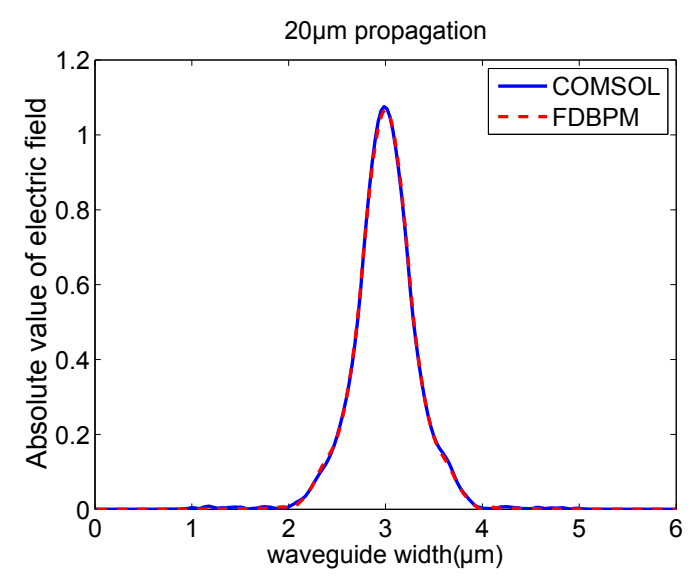

(a)

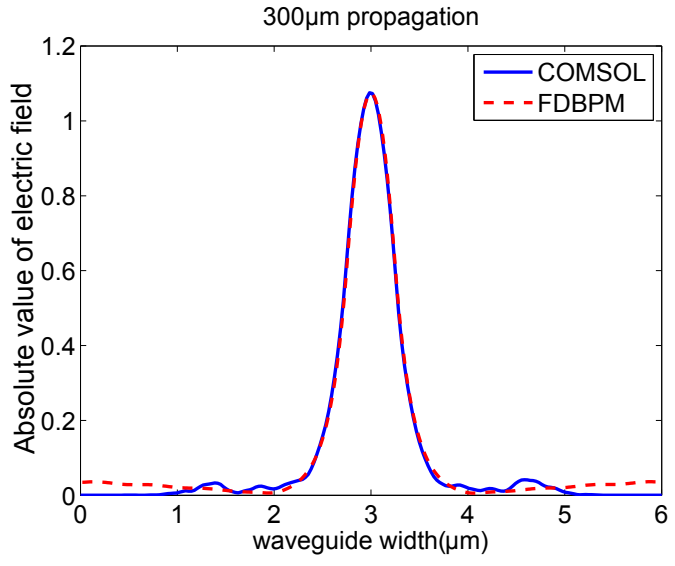

(b)

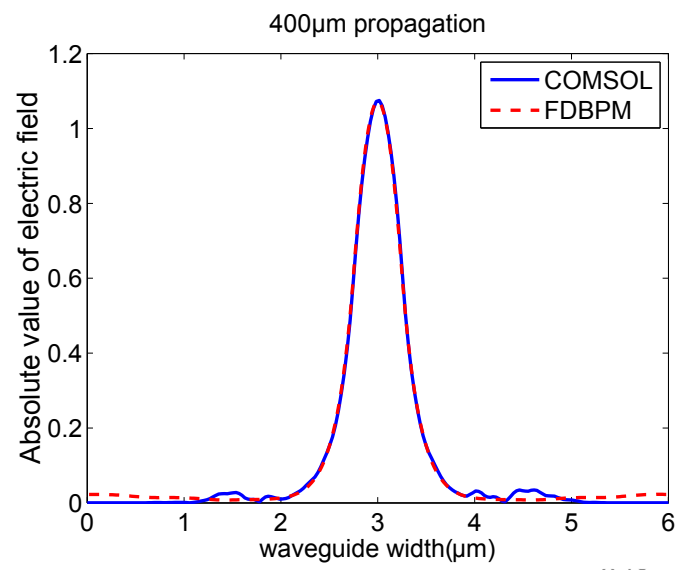

(c)

Figure 2.4: The field amplitudes calculated using FD-BPM and COMSOL for propagation distances of (a) $20 \mu \mathrm{m}$, (b) $300 \mu \mathrm{m}$ and (c) $400 \mu \mathrm{m}$.

In Chapters 3 and 4, it will be shown that the numerical predictions are in good agreement with the experimental measurements. 


\section{Chapter 3}

\section{Fabrication and characterization of the disordered Polymer Optical Fibers}

\subsection{Introduction}

In a theoretical work by P. W. Anderson [1], it was shown that in the presence of disorder in a quantum electronic system, the diffusion process stops and localized electronic states develop. Anderson localization is a wave phenomenon that can also occur for classical waves such as light. Since the theoretical prediction of Anderson localization in optics [3,4], there have been many efforts to realize this phenomenon experimentally with electromagnetic waves $[9,11]$. However, it has been very difficult to achieve strong localization because the optical scattering cross sections are often too small due to the low refractive index contrast of most optical materials. The possibility of observing the Anderson localization in a quasi-two-dimensional disordered optical system with low refractive index contrasts was predicted in Refs. [13,14]. They showed that if the disorder is confined to the transverse plane of a propagating wave 
in a longitudinally invariant medium, the beam can remain confined to a small region in the transverse direction due to strong transverse scattering. Transverse Anderson localization was first observed in two-dimensional waveguides that were created by using interference patterns in a photo-refractive crystal [15]. Fused silica is the other medium that has been used for the observation of transverse Anderson localization [32,33], where disordered waveguides are written using femtosecond pulses along the sample. The refractive index difference of disordered sites in the above mentioned systems are on the order of $10^{-4}$, so the localization radius is quite large. Additionally, the typical waveguides is usually not longer than several centimeters; therefore, they may not be practical for guided-wave applications. We point out that the observation of transverse Anderson localization in a one-dimensional disordered waveguide was reported earlier in Ref. [17].

The optical fiber developed here has several advantages over the previous realizations of transverse Anderson localization for guided-wave applications [20,22]. First, the large refractive difference of 0.1 between the disorder sites of the fiber results in a small localized beam comparable to the beam radius of regular optical fibers. Second, the polymer disordered optical fiber can be made much longer than the disordered waveguides written externally into photo-refractive crystals or fused silica. We were able to observe transverse Anderson localization in a $60 \mathrm{~cm}-$ long fiber [20]. Third, the polymer disordered optical fiber is flexible, making it practical for real world devicelevel applications that rely on the transport of light waves in fibers [24].

In order to fabricate the disordered optical fiber, 40,000 strands of PMMA and 40,000 strands of PS were randomly mixed, where each strand was 8 inches long and $200 \mu \mathrm{m}$ in diameter. The randomly mixed strands were assembled into a square cross-section preform with a side width of about 2.5 inches. The preform was then drawn to a square optical fiber with a side width of about $250 \mu m$ (Fig. 3.1). In order to randomly mix 
the original fiber strands, we spread a layer of PMMA fiber strands on a large table, added a layer of PS fiber strands, and then randomly mixed them together. The procedure was repeated many times until a good random mixture was obtained.

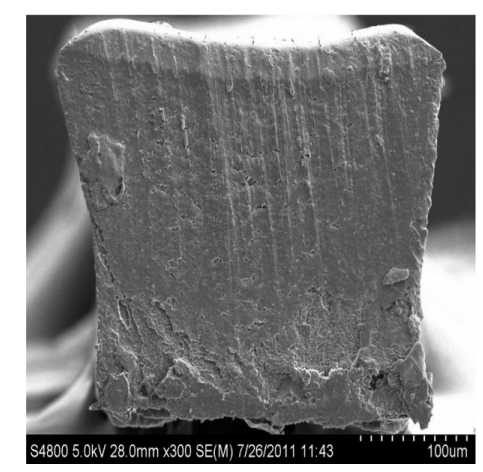

Figure 3.1: SEM image of the polished polymer disordered fiber.

We used a scanning electron microscope (SEM) to image the refractive index profile of the disordered polymer optical fiber. Regular cleaving techniques such as using a sharp heated blade cannot be used to prepare the fiber samples for the SEM imaging of the fiber end to map its refractive index profile, because the blade damages the morphology of the fiber end. Polishing the fiber has a similar detrimental impact on the quality of the fiber end. In order to prepare high-quality samples for the SEM imaging, we submerged each fiber in liquid nitrogen for several minutes and then broke the fiber; if done on enough fiber samples, this method results in a few good fiber pieces (around 15\% success rate) with very high-quality and smooth end surfaces for the SEM imaging. We then used a $70 \%$ ethyl alcohol solution at $65^{\circ} \mathrm{C}$ for about 3 minutes to dissolve the PMMA sites on the fiber end; longer exposure can disintegrate the entire fiber end. We then coated the samples with $\mathrm{Au} / \mathrm{Pd}$ and placed them in the SEM chamber. The zoomed-in SEM image of the disordered polymer optical fiber is shown in Fig. 3.2. The light gray sites are PS and the dark sites are PMMA. The total width of the image is $24 \mu \mathrm{m}$ where the smallest features sizes in this image are $0.9 \mu \mathrm{m}$, corresponding to the individual site sizes of the fiber strands, after the draw 
process.

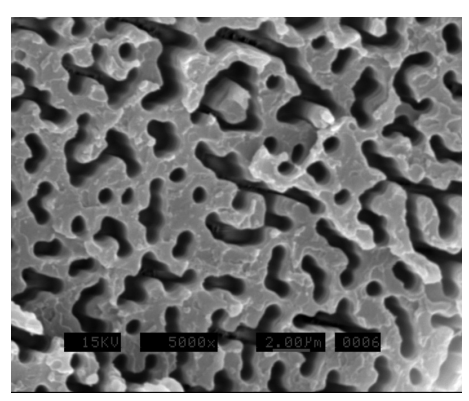

Figure 3.2: Zoomed-in SEM image of the refractive index profile of the disordered polymer fiber. The PMMA sites are in dark color while PS sites are light gray.

In order to characterize the waveguide properties of the disordered optical fiber, we used a He- Ne laser at $633 \mathrm{~nm}$ wavelength. The He-Ne laser is coupled to a single mode SMF630hp optical fiber with a mode field diameter of about $4 \mu \mathrm{m}$, which is then butt-coupled to the disordered polymer optical fiber using a high-precision motorized stage. The output is then imaged on a CCD camera beam profiler using a 40x objective.

In the first set of experiments, we chose 20 different disordered fiber samples, each 5 cm long; the $5 \mathrm{~cm}$ length was chosen to match the propagation length in our numerical simulations. The numerical simulations of the disordered fiber are generally very time consuming, even on a high performance computing cluster with 1100 elements. The full transverse Anderson localization for the wavelength of $633 \mathrm{~nm}$ happens only after about $2.5 \mathrm{~cm}$ of propagation [20,22]; therefore, we decided that the $5 \mathrm{~cm}$ length is sufficient for our purposes. Because of the stochastic nature of the Anderson localization, we needed to repeat both the experiments and the simulations for 100 realizations, in order to collect sufficient statistics to compare the experimental and numerical values of the average beam radius. In practice, 100 different measurements are obtained by taking five spatially separated measurements on each of the 20 different disordered fiber samples. 
It is fairly hard to prepare the disordered polymer optical fibers for measurements, compared with glass optical fibers. For example, one cannot use the advanced cleaving and polishing tools and techniques that are well-developed for standard silica-based fiber. A refined procedure for cleaving and polishing polymer optical fibers has been reported by Abdi et al. [34]; we used their methods with some minor modifications to prepare our fiber samples. In order to cleave a polymer disordered optical fiber, a curved X-Acto blade is heated to $65^{\circ} \mathrm{C}$, and the fiber to $37^{\circ} \mathrm{C}$. The tip of the fiber is aligned on a cutting surface so that a clean, perpendicular cut can be made. The blade is placed on the side of the fiber, and quickly rolled across. The entire cleaving process should be done as fast as possible to ensure that the temperatures of the blade and fiber do not change considerably. After cleaving the fiber and inspecting it under an optical microscope, the fiber end is polished using standard fiber lapping sheets (0.3 $\mu \mathrm{m}$ Thorlabs LFG03P Aluminum Oxide Polishing Paper) to ensure that any minor imperfections are removed. To polish the fiber end, it is held in a pair of tweezers with the tweezers holding the fiber approximately $1.5 \mathrm{~mm}$ away from the end face being polished. The fiber is drawn over the paper in one-inch long figure8-shaped paths, approximately eight times. Polishing the fiber results in smoother edges as inspected under the optical microscope. Moreover, polishing facilitates proper coupling to a localized spot in the fiber, which in turn reduces the attenuation both in the coupling and also in the initial propagation distance before the localized spot is formed. We used a CCD camera beam profiler to image the output beam intensity. The near-field intensity profile was captured using a 40x objective. In order to find the boundaries of the fiber, we saturated the CCD by increasing the power of the incoming light from the SMF630hp fiber. After detecting the intensity profile of the localized beam with respect to the boundaries, we set the CCD beam profiler to the auto-exposure option. We used the image of the intensity profile in order to 
calculate the effective beam radius. In order to remove the effect of the ambient noise, we calibrated our image processing procedure to ensure that we obtain the expected beam radius of the SMF630hp fiber. The average measured value of the beam radius and its variations around the average value agree well with the numerical simulations, as shown in Ref. [20]. The output beam profile in the polymer fiber clearly follows a change in the position of the incident beam as shown in Refs. [22,24]. A comprehensive study of the impact of the design parameters such as the disorder site sizes and the incident wavelength on the beam radius of the localized beam was presented in Refs. [22,35].

\subsection{Procedure}

1. Fabricating a disordered polymer optical fiber:

(a) Spread about 200 of the PMMA strands on a table and spread the same number of PS strands on top of the PMMA. Mix and repack the strands. Repeat this procedure until 40,000 strands of PMMA are randomly mixed with 40,000 strands of PS. A hundred of the original strands of PMMA and PS are shown in Fig. 3.3.

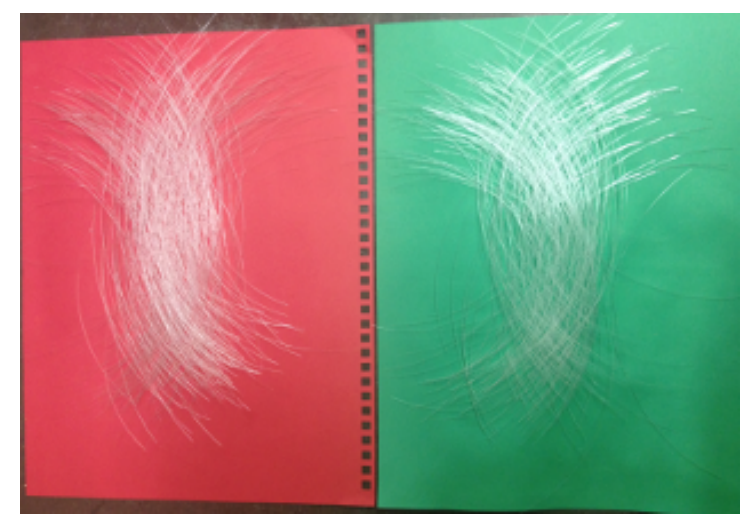

Figure 3.3: Original Strands of PMMA and PS. 
(b) Assemble the randomly mixed strands into a square preform with a side width of about 2.5 inches as shown in Fig. 3.4.

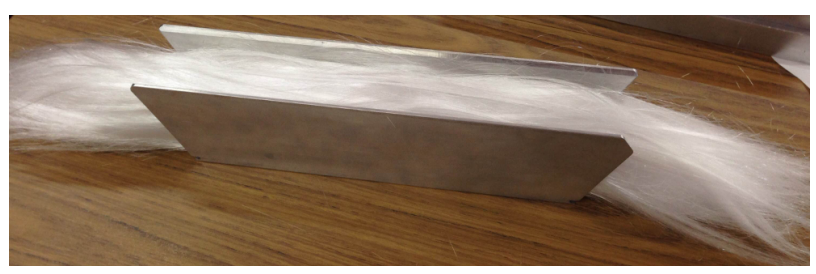

Figure 3.4: Randomly mixed strands of PMMA and PS inside the square preform.

(c) Draw the preform into an optical fiber with a side width of $250 \mu \mathrm{m}$. The preform is drawn at Paradigm Optics Incorporated using their standard procedure [36].

2. Imaging the refractive index profile of the disordered fiber:

(a) Use an SEM such as Topcon ABT, Fig. 3.5(a), to image the refractive index profile of the resulting optical fiber.

(b) Submerge the polymer optical fiber samples in liquid nitrogen for about 10 minutes and then break in half.

(c) Submerge the broken tips of the samples in ethyl alcohol. Keep the temperature of the solution at about $65^{\circ} \mathrm{C}$. Leave the samples in the solution for about 3 minutes, until the ethyl alcohol dissolves the PMMA sites in the fiber.

(d) Coat each sample with a $10 \mathrm{~nm}$-thick layer of $\mathrm{Au} / \mathrm{Pd}$ and place the sample in the chamber of the SEM, Fig. 3.5(b).

3. Preparing the fiber samples for optical characterization:

(a) Prepare $5 \mathrm{~cm}$ long fiber samples. 


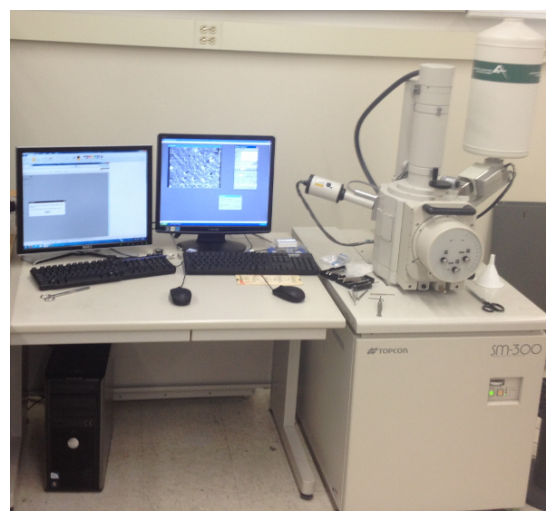

(a)

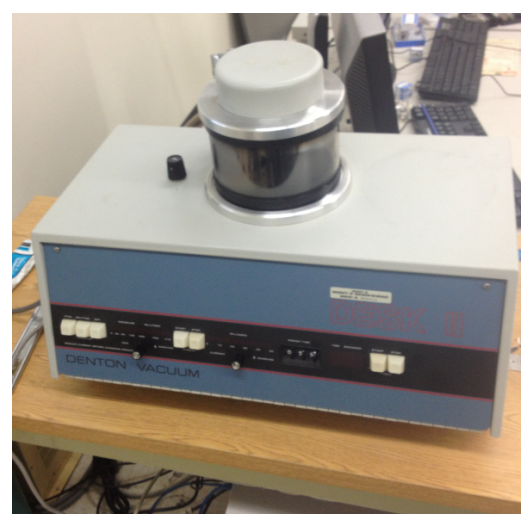

(b)

Figure 3.5: (a) Scanning electron microscope, (b) Sputterer.

(b) Heat a curved blade to $65^{\circ} \mathrm{C}$, and the fiber to $37^{\circ} \mathrm{C}$. Using the right temperatures prevents the deformation of the fiber tip that can occur in the cleaving process.

(c) Align the tip of the fiber on a cutting surface, so that a clean, perpendicular cut can be made. Place the blade on the side of the fiber, and then quickly roll across.

(d) Inspect the fiber tip using an optical microscope to make sure the fiber tip is cleaved perpendicular to the fiber sides. Keep the razor blade at a right angle in the cleaving process to prevent tilting of the tip.

(e) Use a polishing paper such as Thorlabs LFG03P Aluminum Oxide Polishing Paper $(0.3 \mu \mathrm{m})$ to polish the fiber samples. To polish the fiber end, hold it in a pair of tweezers, with the tweezers gripping the fiber approximately $1.5 \mathrm{~mm}$ away from the face being polished. Draw the fiber over the paper in one-inch long figure-8-shaped paths, approximately eight times. Figure8-shaped paths ensure that the whole tip is polished.

4. Characterizing the disordered polymer optical fiber: 
(a) Couple the He-Ne laser into a SMF630hp fiber using a 20x objective and two flat mirrors. Place the flat mirrors on stages with two degrees of freedom. Place the objective on a stage with three degrees of freedom. Initially keep the SMF fiber a distance of $8 \mathrm{~mm}$ away from the objective tip. Using the knobs on the mirror holders and the objective holder, illuminate the laser light to the tip of the fiber. Connect the other side of the SMF to a power meter. Couple power into the SMF using the knobs on the mirror holders as well as the transversal knobs on the objective holder. The efficiency of coupling can be significantly increased by using the longitudinal positioning knob on the objective holder. A coupled power of $1 \mathrm{~mW}$ is enough for the measurements.

(b) Couple the SMF630hp fiber to the polymer optical fiber using Thorlabs MAX343 motorized stage. The motorized stage can be moved in the three Cartesian directions. Using the transversal degrees of freedoms, couple the SMF fiber to the center of the polymer fiber tip. Using the longitudinal displacement of the stage, place the SMF fiber as closely as possible to the polymer fiber. A smaller air gap between the SMF and polymer fiber reduces the expansion of the beam. Place the entire setup on a second motorized stage that moves in the longitudinal direction. The second motorized stage is used for imaging as will be described in 4 (e).

(c) Using an optical microscope and a right-angled mirror, monitor the position of the SMF and polymer fiber to make sure that the SMF is coupled in the center of the polymer fiber, and that the air gap between the two fibers is as small as possible. A small tilt in the polymer fiber tip or deformations in the polymer fiber tip because of cleaving or polishing processes can limit the minimum air gap between SMF and polymer fiber. A small gap between 
the fibers is required because the SMF fiber should be able to move around at the tip of the polymer fiber. Place the SMF at the center of the polymer fiber solely to make the coupling process easier. During the experiment, transversely sweep the incident beam that comes out of the SMF across the tip of the polymer fiber to observe localization in different regions of the polymer fiber.

(d) Use a CCD camera beam profiler to measure the output of the fiber using a 40x objective. First, saturate the CCD camera to monitor the boundaries of the polymer fiber. Using the knobs on the objective holder, make sure that the polymer fiber boundaries can be observed on the CCD.

(e) Use a motorized stage that moves the whole setup (described in 4 (b)) longitudinally, make sure the image on the CCD is focused by moving the setup away or towards the 40x objective while the CCD and objective are fixed. As a metric for focusing, an imaged profile on the CCD should have the minimum size at the focus. A focused image of the beam should not be visually winding.

(f) Move the incident beam at the input tip and measure the output beam intensity for different incident beam positions. Collect data for 5 different positions of the incident beam. Carry out the measurements for 20 fiber samples and collect a total of 100 different measurements.

\subsection{Representative results}

The SEM image of the polished fiber in Fig. 3.1 shows that, for most regions of the fiber tip, the polish quality is good. The SEM image of the fiber samples with their ends dissolved in ethyl alcohol solution, Fig. 3.2, shows the PMMA sites in dark and 
PS sites in gray colors. The SEM image in Fig. 3.2 is zoomed in on a $24 \mu \mathrm{m}$ width of the fiber. For SEM imaging, the fiber samples are coated with a $10 \mathrm{~nm}$ thick layer of $\mathrm{Au} / \mathrm{Pd}$ and imaged using a SEM. The measurement setup used in this experiment is shown in Fig. 3.6. The output beam intensity measured by the CCD beam profiler in a sample of the $5 \mathrm{~cm}$ length is shown in Fig. 3.7. The intensity profile show that the beam is localized in the transverse direction of the disordered fiber. In order to image the intensity profile, the ambient noise correction option of the CCD camera should be on. However, this option may not be completely effective. In order to calculate the total noise level in the intensity profile image, we also imaged the intensity profile of the SMF630hp fiber and the mode field diameter was calculated. For a chosen noise level, the experimental measurement of mode field diameter is matched with the reported manufacturer data. The same value of noise level needs to be used for interpretation of Figs. 3.7. One hundred different intensity profiles of the localized beams are measured by moving the input SMF630hp fiber in the transversal positions at the coupling with the polymer fiber for 20 different samples. One hundred measurements of the beam profiles are averaged to show the transverse Anderson localization in a disordered optical fiber as presented in Ref. [20].

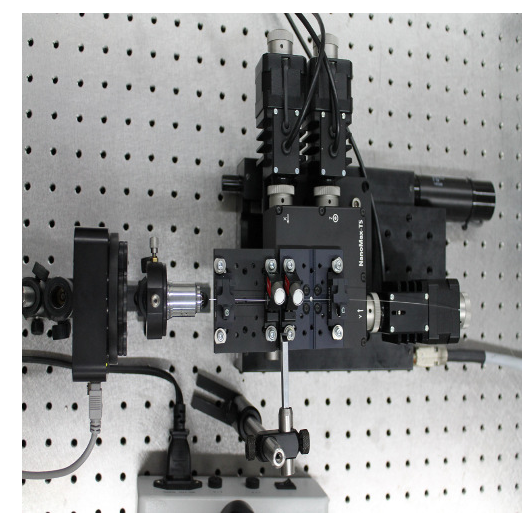

Figure 3.6: The coupling and detection sections of the experimental setup. 


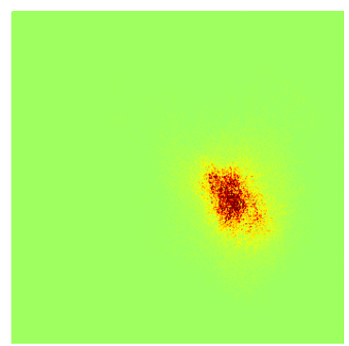

(a)

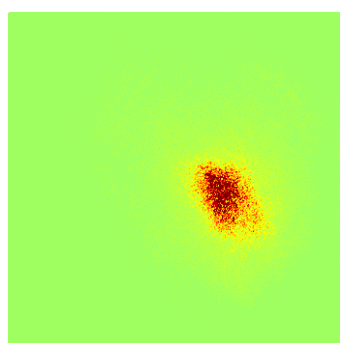

(b)

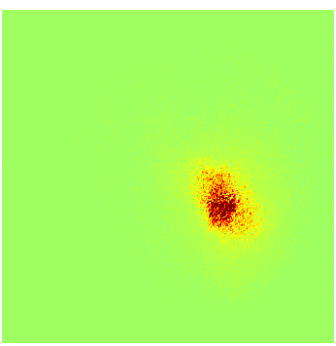

(c)

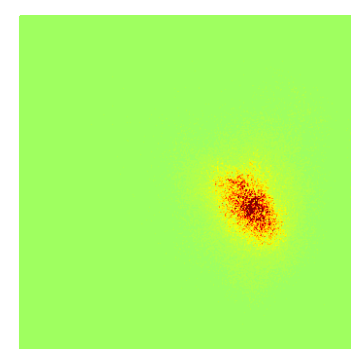

(d)

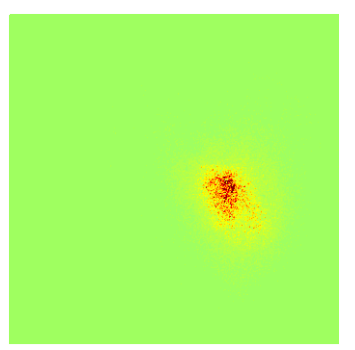

(e)

Figure 3.7: (a)-(e) Intensity profiles for five different locations of the input beam.

\subsection{Discussion}

In the fiber draw process, the refractive index profile does not remain constant for more than a meter, both because of the cross-overs of the original fiber strands and also because of the variations of the fiber diameter in the draw process. We expect that a more stable draw process will help to fabricate an optical fiber that is invariant over longer fiber lengths compared with that reported here. In preparing a sample for the SEM imaging of the fiber tip, we need to ensure that the sample remains in the $70 \%$ ethyl alcohol solution for a long enough time (3 minutes) and remains at the right temperature $\left(65^{\circ} \mathrm{C}\right)$. If the sample remains in the ethyl alcohol solution much longer than the 3 minutes required to etch away the top layer of PMMA, the fiber tip can disintegrate. In butt-coupling the SMF630hp fiber to the polymer fiber, it is important that the incident fiber is as close as possible to the polymer fiber to avoid substantial diffractive expansion of the beam before it reaches the disordered 
fiber. We also need to use an index-matching fluid to reduce the scattering of light at the coupling. We should note that moving the incident field across the end face of the disordered fiber changes the position of the localized beam at the output. In different regions of the polymer fiber, we observe variations in the localized beam radius, as expected from the statistical nature of the Anderson localization. Some of this variation can also be attributed to the quality of polish of the fiber end. As the SEM image of the polished fiber shows, the quality of the polish is not the same in all regions of the fiber end. Because of this limitation, we used the smallest localized spot that we could find across the fiber end for each experiment and then carried out the remaining measurements in the neighborhood of the best localized spot. Removing the ambient noise is crucial for calculating the beam radius of the localized beam. If not removed, the ambient noise can result in an error in the calculation of the beam radius of the CCD beam profiler images. We calibrated our analysis to ensure that we obtain the correct value of the beam diameter of about $4 \mu \mathrm{m}$ for the SMF630hp fiber at $633 \mathrm{~nm}$ wavelength. Numerical modeling of light localization in polymer disordered fibers in Refs. [20,22] for lossless materials shows that the wave can totally be confined in the transverse directions of the fiber without any attenuation in power. On the other hand, the material absorption in our fibers is considerable and the fiber attenuation is at the level of $0.5-1.0 \mathrm{~dB} / \mathrm{cm}$. We expect the loss to be considerably lower in silica-based disordered fibers. In the future, we anticipate improving the loss properties of disordered fibers by improving the fabrication procedure (e.g. a more stable draw process) and also using lower-loss components. The ideal disordered optical fiber will be composed of glass with random air voids at $50 \%$ ratio. As we have shown in Ref. [22], we expect that the larger difference in the refractive indices of two materials results in reduced variations of the localized beam radius. We recently presented our first results in glass optical fiber with disordered air void sites in Ref. [23] 
and anticipate future progress in glass-based disordered fibers. 


\section{Chapter 4}

\section{Transverse Anderson localization in the disordered polymer optical fiber}

\subsection{Introduction}

Anderson localization is the absence of diffusive transport of waves in a highly disordered medium $[1,3-5,17,37]$. In order to observe Anderson localization, the disorder must be strong enough such that the wave scattering transport length $l^{\star}$ becomes of the order of the wavelength i.e. $k l^{\star} \sim 1$, where $k$ is the effective wavevector in the medium [7]. While it is notoriously difficult to satisfy this condition and observe strong localization effects for light in three dimensions, the required conditions are considerably relaxed in two dimensional systems $[13,14]$. Two-dimensional disordered systems are always localized and the localization length $\xi$, which is the effective radius of the localized beam is related to $l^{\star}$ by $\xi=l^{\star} \exp \left(\pi k_{T} l^{\star} / 2\right)$. If the randomness in the refractive index profile is only limited to the transverse plane of an optical wave, the effective transverse component of the wavevector $k_{T}$ is 10-100 times smaller than 
$k$, therefore, even small disorder (i.e. large $l^{\star}$ ) can readily result in a beam diameter, which is smaller than the transverse dimensions of the system.

Transverse localization of light was recently observed experimentally by Segev's group [15] in a two-dimensional photonic lattice with random refractive index fluctuations induced on a photo-refractive crystal using an optical interference pattern. This pioneering experiment clearly proves the transverse Anderson localization concept; however, the photo-induced refractive index fluctuations are of the order of $10^{-4}$, resulting in a large localization length (effective beam radius). Moreover, such a low index contrast is responsible for large fluctuations in the localization length among different realizations of the random refractive index profile. In order to use transverse Anderson localization for reliable optical transport, larger (and permanent) refractive index fluctuations are desired.

\subsection{Disordered polymer optical fibers}

Here, we introduce an optical fiber with a binary compound which has permanent refractive index fluctuations on the order of 0.1 and observe transverse localization of light with an effective propagating beam diameter which is comparable to that of a typical index-guiding optical fiber. The large refractive index fluctuation significantly reduces the variations among different realizations of the random optical fiber, as is required for device applications.

Our disordered optical fiber is based on the structure proposed and numerically analyzed by De Raedt et al., where the refractive index is invariant in the longitudinal z-direction, but is randomly changing in the two "transverse" directions [13]. For the numerical simulations, we use an idealized structure, where we start with a square 
transverse geometry for the optical fiber of side dimension $L$. We then create a square grid of mesh size $d$ covering the larger square; if $L=N \times d$, the total number of cells covering the transverse profile of the fiber is $N^{2}$. The refractive index of each cell can be either $n_{1}$ or $n_{2}$. We define $p$ as the probability of each cell having a refractive index $n_{1}$ and assume without loss of generality that $n_{1}<n_{2}$. Therefore, $p$ can be regarded as the filling fraction of the low-index material in the higher index host medium. In Fig. 4.1(a), we show a sample refractive index profile that we have used for our simulations; the black regions have refractive index $n_{1}$, while the white regions have refractive index $n_{2}$.

Our fabricated fiber closely resembles the idealized structure discussed above. The high and low refractive index regions in the optical fibers were fabricated from polystyrene (PS) with refractive index of 1.59 and polymethyl methacrylate (PMMA) with refractive index of 1.49. 40,000 pieces of PMMA and 40,000 pieces of PS fibers were randomly mixed; each fiber was 8 inches long with an approximate diameter of $\sim 200 \mu \mathrm{m}$. The mixture was fused together and redrawn to a fiber with a nearly square profile and approximate side width of $250 \mu \mathrm{m}$ [36]. While some of the original randomly mixed fibers might have crossed over each other during the assembly and redraw process, the large redraw ratio guarantees that the refractive index profile remains relatively unchanged along the fibers in our experiments.

Our fabricated structure corresponds to the theoretical parameters of $p=50 \%$ (below percolation threshold $59.27 \%$ of a square lattice) and $d \sim 0.9 \mu \mathrm{m}$, used in our simulations. In Fig. 4.1(b), the scanning electron microscope (SEM) image of a polished optical fiber cross section is shown; the high and low refractive index regions are not distinguishable in this picture. The side width of the fiber in this SEM image is approximately $250 \mu \mathrm{m}$. We also exposed the tip of the optical fiber to $70 \%$ ethanol solvent at $60^{\circ}$ Celsius for about three minutes to dissolve the PMMA. The SEM im- 


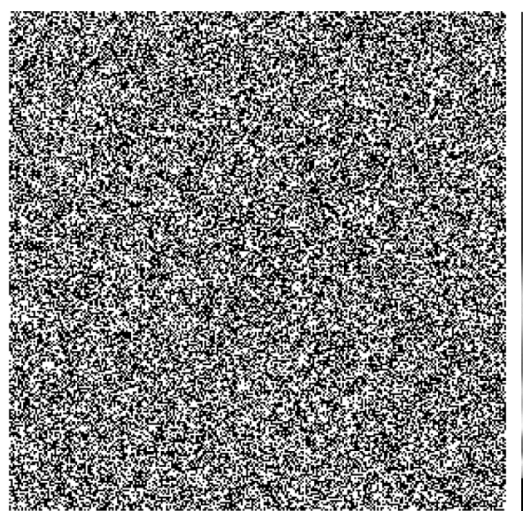

(a)

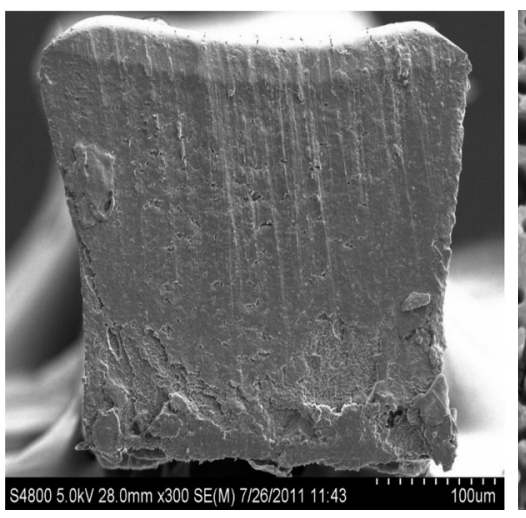

(b)

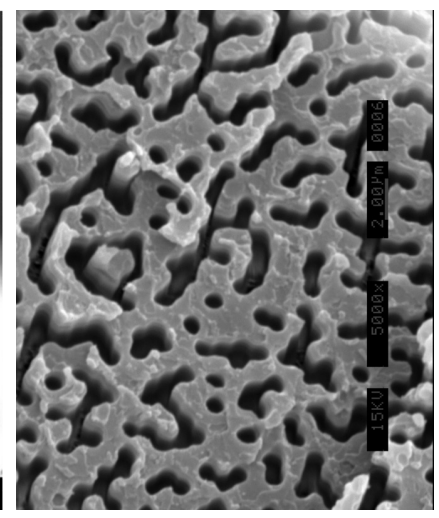

(c)

Figure 4.1: a) A sample refractive index profile that we have used for our simulations $(p=50 \%)$; the black regions have refractive index $n_{1}=1.49$, while the white regions have refractive index $n_{2}=1.59$, b) SEM image of a polished fiber tip: the high and low refractive index regions are not distinguishable in this SEM image, and c) SEM image of a fiber tip exposed to the solvent, where the feature sizes in (c) are substantially smaller than the localized beam diameter, as shown in Fig. 4.3.

age of an ethanol-etched fiber sample with the side widths of $250 \mu \mathrm{m}$ is shown in Fig. 4.1(c), where the PMMA (constituent with the lower refractive index) regions are darker in color, and PS regions are lighter. The total viewable width of the zoomedin SEM image in Fig. 4.1(c) is $24 \mu \mathrm{m}$ in the vertical direction. We will later show that the feature sizes in Fig. 4.1(c) are substantially smaller than the localized beam diameter. Experimental observation of transverse Anderson localization In order to investigate the guidance and localization properties of the Anderson localized optical fiber (ALOF), we directly launch a $632.8 \mathrm{~nm}$ beam from a single mode optical fiber (SMF, 630hp from Thorlabs, $2 \pm 0.25 \mu \mathrm{m}$ core radius) into the Anderson-localized optical fiber (ALOF) using the butt-coupling method. The near-field output beam from the ALOF is imaged onto a CCD camera beam profiler using a 40x objective. In Fig. 4.2(a), we show the measured near-field intensity profile of the optical beam after propagating through a $60 \mathrm{~cm}$ long ALOF, where the beam remains clearly localized. The large variations in the side width of the optical fiber in the draw process made it difficult for us to do reliable measurements over longer samples of the ALOF; however, 
we expect that our observations can be extended to much longer ALOF by using a more stable draw process and using low attenuation glasses. In order to compare our experimental observations to numerical simulations, we decided to carry out our comparisons over shorter samples of fiber $(5.5 \mathrm{~cm})$. While the localization clearly holds for at least $60 \mathrm{~cm}$, the beam reaches its final localized radius after propagating for $\sim 2 \mathrm{~cm}$; therefore, we chose shorter samples for comparison, in order to somewhat reduce the massive computational effort needed to carry out these simulations.

The output beam profile from a $5.5 \mathrm{~cm}$ long sample ALOF is shown in Fig. 4.2(b). The beam is visually localized relative to the boundaries of the fiber which are approximately the boundaries of the figure. The SMF is scanned across the input tip of the ALOF, using a high-precision motorized stage with resolution of $5 \mathrm{~nm}$. The localized spot at the output tip of the ALOF clearly follows the tip of the SMF, as the SMF is scanned across the input tip of the ALOF. Fig. 4.2(c) shows the intensity profile of a typical ALOF resulting from the simulations that can be compared with our experimental measurements in Fig. 4.2(b). We note that in the absence of disorder, the beam profile would fill the entire cross section of the fiber after only a few millimeters.

We repeated our near-field measurements for 20 pieces of ALOF, each nearly $5.5 \mathrm{~cm}$ long. For each fiber, we scanned the SMF across the input tip of the ALOF and visually found the best localized spot and measured the near-field beam profile. Although the ALOF tip is carefully polished and inspected for each experiment, the surface quality is lower than what is routinely achievable for glass optical fibers; therefore, the localized spot slightly varied across the tip of the ALOF. In order to take this variation into consideration, we moved the SMF tip by $10 \mu \mathrm{m}$ to the left, right, up, and down, relative to the original spot, and measured the four new near-field beam profiles. The mean effective beam radius for the 100 measured near-field beam intensity profiles was 


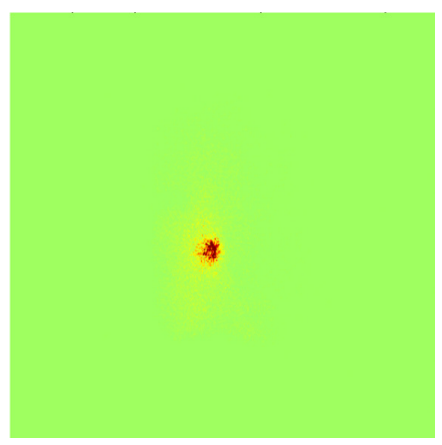

(a)

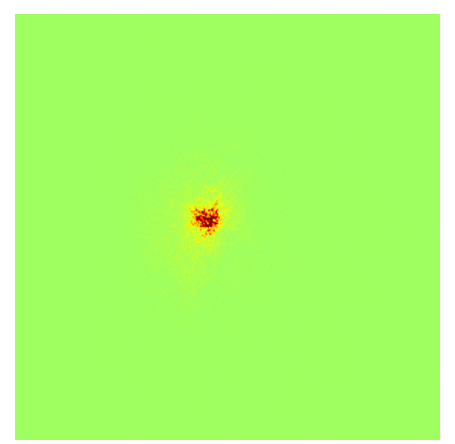

(b)

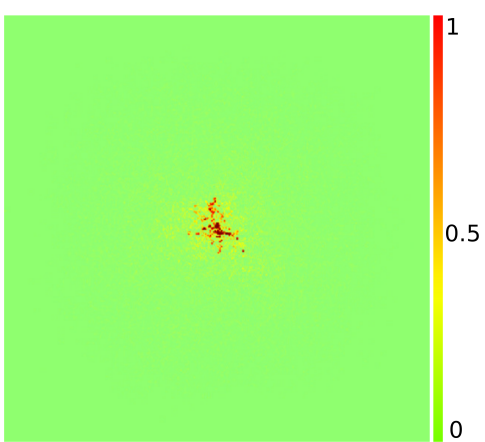

(c)

Figure 4.2: a) Near-field intensity profile after $60 \mathrm{~cm}$ of propagation from experiment, b) Near-field intensity profile after nearly $5 \mathrm{~cm}$ of propagation in a sample ALOF from b) experiment and c) simulation. For comparison, we note that the total side width of each figure is $250 \mu \mathrm{m}$.

calculated to be $\xi_{\text {avg }}=31 \mu \mathrm{m}$, with a standard deviation $\sigma_{\xi}=14 \mu \mathrm{m}$. The result is shown in Fig. 4.3, where the region corresponding to $\xi_{\mathrm{avg}} \pm \sigma_{\xi}$ is highlighted in green.

\subsection{Comparing the experimental measurements with the theoretical calculations}

In Fig. 4.3, we show the results of our direct numerical simulation of our experiment and plot $\xi(z)$ as a function of the propagation distance along the fiber. We numerically solve the paraxial approximation to the Helmholtz equation, using the finite difference beam propagation method (FD-BPM) [26]. The effective beam radii $\xi$ for both experimental results and numerical simulations are calculated by the variance method [13].

The results in Fig. 4.3 are averaged on an ensemble of 100 separate simulations, where the profile of each element of the ensemble is randomized differently corresponding to a fixed filling fraction of $p=50 \%$. The region corresponding to $\xi_{\text {avg }} \pm \sigma_{\xi}$ is highlighted 


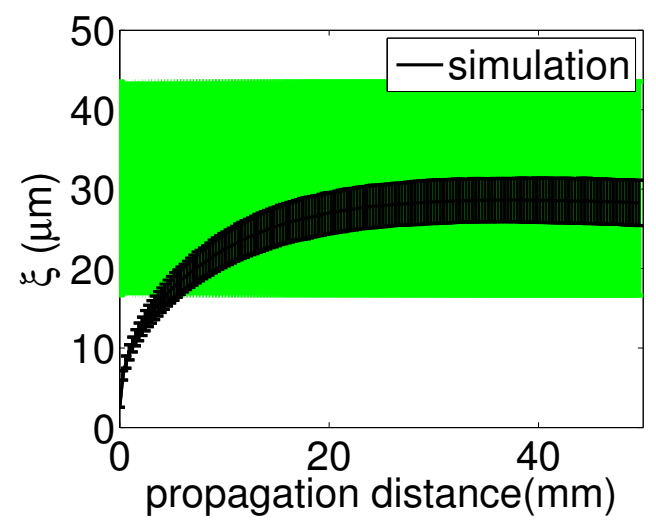

Figure 4.3: The region highlighted in green corresponds to one standard deviation in each direction around the average experimental measurement of the localization length parameter represented by $\xi_{\text {avg }} \pm \sigma_{\xi}$. The measurements are carried over fibers each with an approximate length of $5.5 \mathrm{~cm}$. The region highlighted in black corresponds to theoretical simulation of the effective beam radius $\xi_{\text {avg }} \pm \sigma_{\xi}$ as a function of propagation distance.

in black, where the average localization length $\xi_{\text {avg }}$ and standard deviation $\sigma_{\xi}$ are calculated over the 100-element ensemble for the field profile after $5 \mathrm{~cm}$ of propagation. The input Gaussian beam initially undergoes diffusive broadening [13, 15], until its effective radius becomes of the order of the localization length, after which further expansion is halted and the beam remains localized. We remind that each individual realization of disordered fibers for a given value of $p=50 \%$ will have a different refractive index profile drawn from the distribution, so that the effective beam radius (and the localization length) always fluctuate about the average. The error bars signify the expected variation in the effective beam radius among the elements of the "statistically" identical ensemble. There is reasonable agreement between the theoretical simulations and experimental results. While the average beam radius $\xi_{\text {avg }}$ is almost the same in theory and experiment, the variation in the experimental measurements of the beam radius is larger than the variation in the theoretical results. The difference can be attributed to the variations in the side width of the optical fiber in the draw process. The small size of the simulation error bars indicates that the ensemble averaging is hardly necessary in here, and each individual element of the ensemble should 


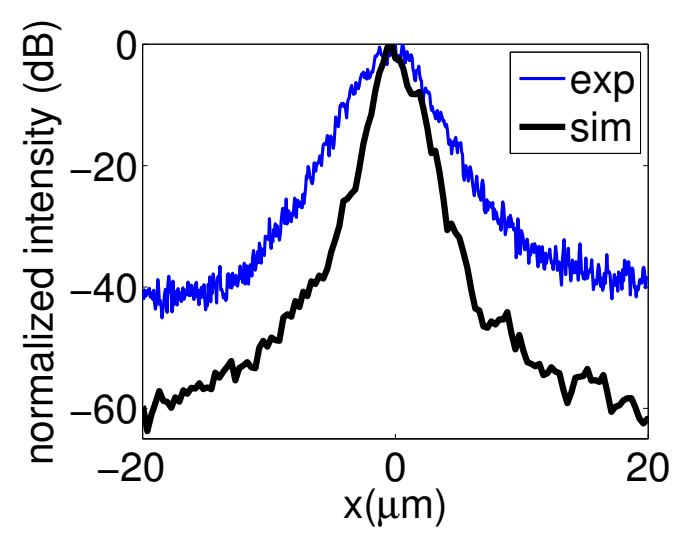

Figure 4.4: Cross section of the intensity profile of the localized beam averaged over 100 samples of raw data from simulations and 100 samples from experiments in dB units.

be practically equivalent to the ensemble average. This "self-averaging" behavior is generally obtained from the theory of wave localization under appropriate conditions (see for example Refs. [38,39]). The "self-averaging" behavior does not hold well when the refractive index contrast is too small as in the case of ref. [15], and wave localization is only meaningful in a statistical averaging sense. In Fig. 4.4, we plot a cross section of the intensity profile of the localized beam, averaged over 100 samples in our experiments and simulations at the $5 \mathrm{~cm}$ propagation length. The exponentially decaying tails of the average intensity clearly illustrate the transverse Anderson localization of the beam. The difference between simulation and experiment is caused by the larger variation in the experimental results (see Fig. 4.3), and also the noise in the CCD beam profiler at low intensities.

\subsection{Localized modes in the disordered polymer opti- cal fiber}

We experimentally and numerically showed that the longitudinal wave propagation in the disordered polymer optical fiber is due to the transverse Anderson localization of 
light. The exponential decay of the averaged intensity profile and also the robust beam radius of the propagating beam for a long propagation distance are the characteristics that show the robust transverse Anderson localization of light in the disordered polymer optical fibers. In this section, we numerically show the origin of the transverse Anderson localization in the disordered polymer fiber by calculating the fully-vectorial eigenmodes. Fully-vectorial eigenmodes of Maxwell's equations with periodic boundary conditions were computed by preconditioned conjugate-gradient minimization of the block Rayleigh quotient in a planewave basis, using a freely available software package [21]. For a set of twelve out-of-plane wavevectors, $\mathrm{K}_{z}$, fifty different eigenmodes are calculated and the results are presented in Fig. 4.5. Different values of the $\left(\lambda, \mathrm{K}_{z}, \mathrm{n}_{\text {eff }}\right)$ are related to the transversely localized modes at different regions of the disordered fiber.

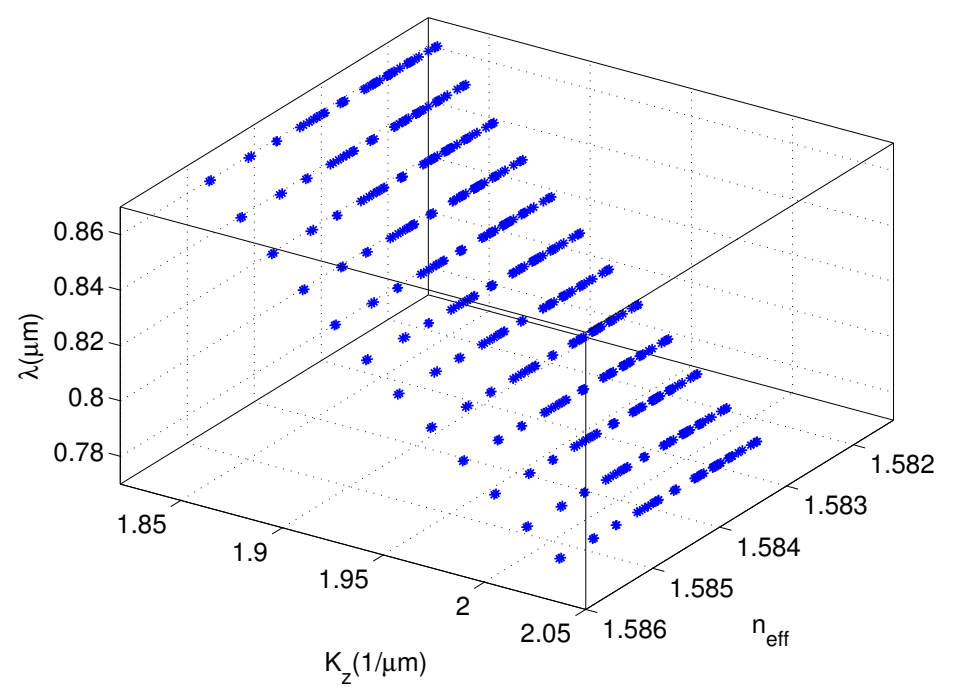

(a)

Figure 4.5: The 3-dimensional plot of the wavelength-wavevector-effective index $\left(\lambda-\mathrm{K}_{z}-\mathrm{n}_{\mathrm{eff}}\right)$ for 50 eigenmodes.

The intensity profiles of a few transversely localized modes at different regions of the 
fiber are shown in Fig. 4.6.

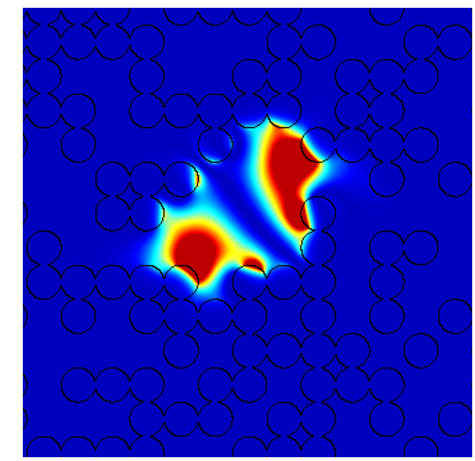

(a)

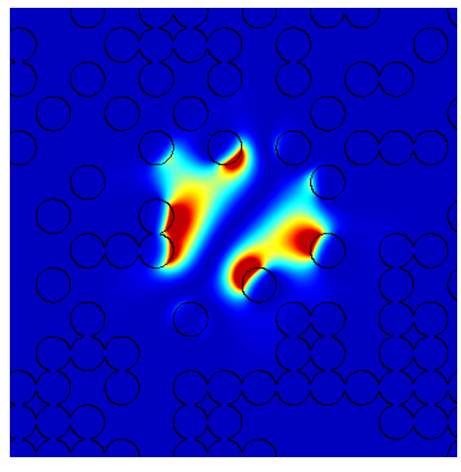

(b)

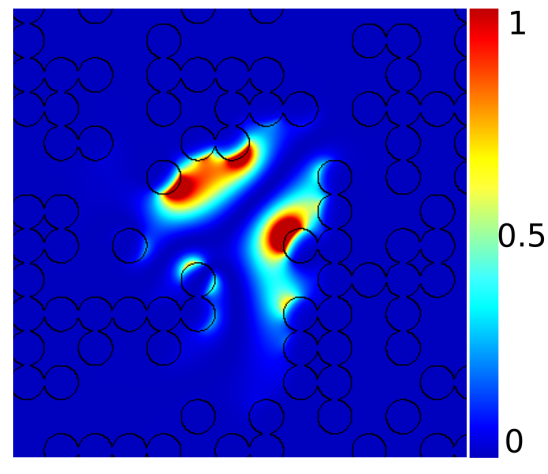

(c)

Figure 4.6: The intensity profiles of the localized modes for different values of $\mathrm{n}_{\text {eff }}$ and at different regions at the tip of the fiber. The black circles indicate the PMMA sites with the refractive index of 1.49. The intensity profiles are zoomed in square regions with the side widths of $12 \mu m$.

To generate a disordered refractive index profile for the modal calculations, we start with a square transverse geometry for the optical fiber. We then create a periodic square lattice of the cylindrical sites with the diameters of $d=0.9 \mu m$ and the periodicity of $0.9 \mu \mathrm{m}$ covering the whole tip of the square fiber; the refractive index of the sites has been chosen randomly to be $\mathrm{n}_{1}=1.49$ (PMMA) or $\mathrm{n}_{2}=1.59$ (PS) with equal probability. The diameter $(\mathrm{d}=0.9 \mu \mathrm{m})$ of the sites was taken from the SEM image of the tip of the disordered polymer optical fiber.

In order to accomplish the numerical calculations in a reasonable time, the width of the square fiber was chosen $120 \mu \mathrm{m}$ and only 50 transversely localized eigenmodes for each $\mathrm{K}_{z}$ are calculated. The width of the strongly localized modes are much smaller than the width of the simulation window, so the periodic boundaries would not impact our calculations. 


\subsection{Conclusion}

In conclusion, we have taken a step towards utilizing Anderson localization in a device application. In addition to the practical importance of the device-level implementation of the localization of light, the availability of well-established fiber-optic characterization techniques and tools provides a unique opportunity to unwrap the complexity of wave transport and localization in future studies. 


\section{Chapter 5}

\section{Effects of design parameters on the}

\section{light localization in the Anderson localized optical fibers}

\subsection{Introduction}

The possibility of micron-scale transverse confinement of light and its extremely lowloss propagation in optical fibers provides a robust platform to explore many new ideas in photonics as well as in other branches of science [40]. The 1996 introduction of photonic crystal fibers [41] was a revolutionary step towards a much greater control over the refractive index profile, resulting in the endlessly single-mode fibers [42], exotic dispersion properties [43], highly nonlinear fibers for supercontinuum generation [44], and bandgap fibers [45], among many others. The framework of the geometrical symmetries of fibers (or lack thereof) may guide its future progress as much as it has shaped its past and provide clues to what is yet to emerge in new scientific findings 
and applications of optical fibers. Traditional optical fibers are symmetric in both longitudinal and angular cylindrical coordinates with a broken radial symmetry required for light confinement. Further symmetry breaking has resulted in novel phenomena, such as polarization maintaining fibers, which break axial rotational symmetry, fiber Bragg gratings, which break longitudinal symmetry, or photonic crystal fibers where the angular symmetry is reduced to at most discrete rotations. It is of no coincidence that symmetry breaking plays a fundamental role at the heart of other scientific disciplines as well [46]. One can argue that besides aesthetics, symmetric structures are generally easier to study, model, and fabricate [41,47]. However, neither the guiding nor even the bandgap properties in photonic crystal fibers require periodicity at the fundamental level.

Disordered structures possess exotic universal physical characteristics, which sets them apart from deterministic structures [1]. For example, the propagation of light in highly disordered media can lead to strong confinement due to the Anderson localization effect [3-6]. Anderson localization has been the subject of intense investigation in various quantum and classical wave disordered systems over the years. There has been considerable progress during the past few years in research on the localization of light waves [6].

In order to observe Anderson localization, the disorder must be strong enough such that the mean free path for wave scattering $l^{\star}$ becomes of the order of the wavelength i.e. $k l^{\star} \approx 1$, where $k$ is the effective wavevector in the medium. This is known as the Ioffe-Regel condition [7]. The Ioffe-Regel condition is very difficult to satisfy in three dimensional optical systems; however, the required conditions are considerably relaxed in two dimensional systems where strong localization effects for light can be readily observed. This interesting fact was pointed out in 1989 by De Raedt et al. [13] who proposed and numerically confirmed "transverse localization" of light in an optical 
system which is uniform in one ("longitudinal") direction but contains disorder in the two "transverse" directions.

Two-dimensional disordered systems are always localized and the localization length $\xi$ is given by $\xi=l^{\star} \exp \left(\pi k_{T} l^{\star} / 2\right)$ (see $\left.[13,15,48]\right)$. In particular, if the randomness in the refractive index profile is only limited to the transverse plane of a propagating optical wave, $k_{T}$ is the effective transverse component of the wavevector and is 10-100 times smaller than $k$; therefore, even small disorder (i.e. large $l^{\star}$ ) can readily result in an effective localized beam radius $(\xi)$, which is smaller than the transverse dimensions of the system. De Raedt et al. [13] observed that a narrow beam entering a medium with transverse disorder undergoes initial diffusive broadening until its radius becomes of the order of the localization length, after which it propagates in a localized transverse profile. Transverse localization of light for the first time was observed experimentally by Segev's group in a two-dimensional photonic lattice with random refractive index fluctuations induced on a photo-refractive crystal using an optical interference pattern [15]. While this pioneering experiment clearly proves the transverse Anderson localization concept as introduced by De Raedt et al. [13], the photo-induced refractive index fluctuations are of the order of $10^{-4}$, resulting in a large localization length (effective beam radius or localization radius). Moreover, such a low index contrast is responsible for large fluctuations in the localization length among different realizations of the random refractive index profile. Transverse Anderson localization in 1D lattices is investigated both numerically in Ref. [49] and experimentally in Refs. [17,32,50], in great detail. In Ref. [17], the disordered structure is a 1D lattice of coupled waveguides on an AlGaAs substrate; and in Refs. [32,50], the waveguides are inscribed using ultrafast lasers inside transparent glass. In a recent work, we introduced an optical fiber with a binary compound with permanent refractive index fluctuations on the order of 0.1 and observed transverse localization of light with an effective propagating beam 
diameter, which was comparable to that of a typical index-guiding optical fiber [20]. We used a disordered polymer optical fiber to carry out our experiments and observed transverse localization of light for fibers as long as $\approx 60 \mathrm{~cm}$; however, longer propagation was affected by large longitudinal variations of the fiber due to the draw process. We made sure that all of the samples selected for our experiments are invariant along the length by inspecting their side width under an optical microscope.

In order to manage and use transverse Anderson localized optical fibers for the device applications, it is of great importance to understand the effect of different optical and geometrical parameters of the fiber on the beam and propagation properties of the localized light, especially on its effective beam radius. Here, we investigate the impact of the disorder site size, fill-fraction, and refractive index contrast on the beam radius of the localized propagating beam in the Anderson localized optical fiber. We mainly focus our studies on design variations of the polymer fiber we reported in Ref. [20]. In particular, we will compare our numerical results on the impact of different disorder site sizes with experimental measurements. However, we will show that our general observations are applicable to a wide range of parameters of interest for applications of transverse Anderson localized optical fibers.

In order to experimentally investigate the impact of the disorder site size, we have chosen the same polymer fibers that we used in Ref. [20], but now drawn to different side widths (fiber profiles are nearly rectangular). The fiber side widths studied in here are approximately $150 \mu m$ and $250 \mu m$, resulting in approximate site sizes of $0.6 \mu \mathrm{m}$ and $0.9 \mu \mathrm{m}$. For numerical investigations, we use the finite difference beam propagation method (FD-BPM) and calculate the beam radius of the localized light in the Anderson localized optical fibers. We will also investigate numerically the effect of different fill-fractions and different refractive index contrasts on the beam radius. Finally, we will compare some of our results with those obtained for a large index 
contrast created in a glass version of the disordered optical fiber where the random air voids create a much larger index contrast. Our observations for the glass version of the disordered fiber, especially the small localized beam radius and the reduced boundary effects, in addition to the intrinsically low material loss, may provide an avenue for potential applications in short-haul optical fiber communications.

\subsection{Anderson localized optical fiber}

Our disordered optical fiber is based on the structure proposed and numerically analyzed by De Raedt et al., where the refractive index is invariant in the longitudinal z-direction, but is randomly changing in the two "transverse" directions [13]. For the numerical simulations, we follow the same procedure that we used in our recent publication [20], the details of which are repeated below. We use an idealized structure, where we start with a square transverse geometry for the optical fiber of side dimension $L$. We then create a square grid of mesh size $d$ covering the larger square; if $L=N \times d$, the total number of cells covering the transverse profile of the fiber is $N^{2}$. The refractive index of each cell can be either $n_{1}$ or $n_{2}$. The random numbers are drawn from a uniform distribution. We define $p$ as the probability of each cell having the refractive index $n_{1}$ and assume without loss of generality that $n_{1}<n_{2}$. Therefore, $p$ can be regarded as the fill-fraction of the low-index material in the higher index host medium.

In order to fabricate the disordered fiber, we follow the procedure we reported recently in Ref. [20]. We randomly mixed 40,000 pieces of Poly(methyl methacrylate) PMMA fibers with refractive index of 1.49 and 40,000 pieces of Polystyrene (PS) fibers with refractive index of 1.59; each fiber was 8 inches long with an approximate diameter of $200 \mu \mathrm{m}$. The mixture was fused together and redrawn to a fiber with a nearly square 
profile and different values of side width, including fibers with approximate side width of $150 \mu \mathrm{m}$ and $250 \mu \mathrm{m}$.

Our choice of the fill-fraction parameter $p=50 \%$ is optimal, in order to obtain the minimum localization length in these fibers. We note that $p=50 \%$ is below the percolation threshold $59.27 \%$ (of a square lattice); therefore, the host material with the higher refractive index $n_{2}$ remains generally connected in the long range. Above the percolation threshold, disconnected clusters of the higher index material form, which can act as individual waveguides; in this case index-guiding by these higher-index localized clusters can mask any effects that can be attributed to Anderson localization and the optical field can be localized in a trivial sense.

In Fig. 5.1(a), we show an example cross section of a fiber with site size of $0.9 \mu \mathrm{m}$ used for our simulations, corresponding to a fiber of side width equal to $250 \mu \mathrm{m}$. However, the square only shows a $24 \mu m \times 24 \mu m$ region of this fiber, in order to clearly capture the details of the structure used for our simulations. Similarly, SEM images of the fibers with side width of $150 \mu m$ and $250 \mu m$ over a $24 \mu m \times 24 \mu m$ square are shown in Figs.5.1(b) and (c), respectively. As it is apparent in the SEM images in Figs.5.1(b) and (c), the fiber with the larger side width $250 \mu \mathrm{m}$ has larger feature sizes, $(0.9 \mu \mathrm{m}$ versus $0.6 \mu \mathrm{m}$ ), compared with those of the fiber with the side width of $150 \mu \mathrm{m}$.

In order to carry out the experiment to observe the transverse Anderson localization, we coupled a He-Ne laser at the wavelength of $632.8 \mathrm{~nm}$ to a single mode fiber with the beam radius of $2 \mu \mathrm{m}$ and butt-coupled the single mode fiber to the Anderson localized optical fiber. The distance between the Anderson localized optical fiber and the single mode fiber is around $10-20 \mu \mathrm{m}$, depending on how cleanly we can cleave and prepare the Anderson localized optical fiber. The polymer fibers are cleaved according to the recipe suggested in Ref. [34], where we modified their procedure to accommodate 

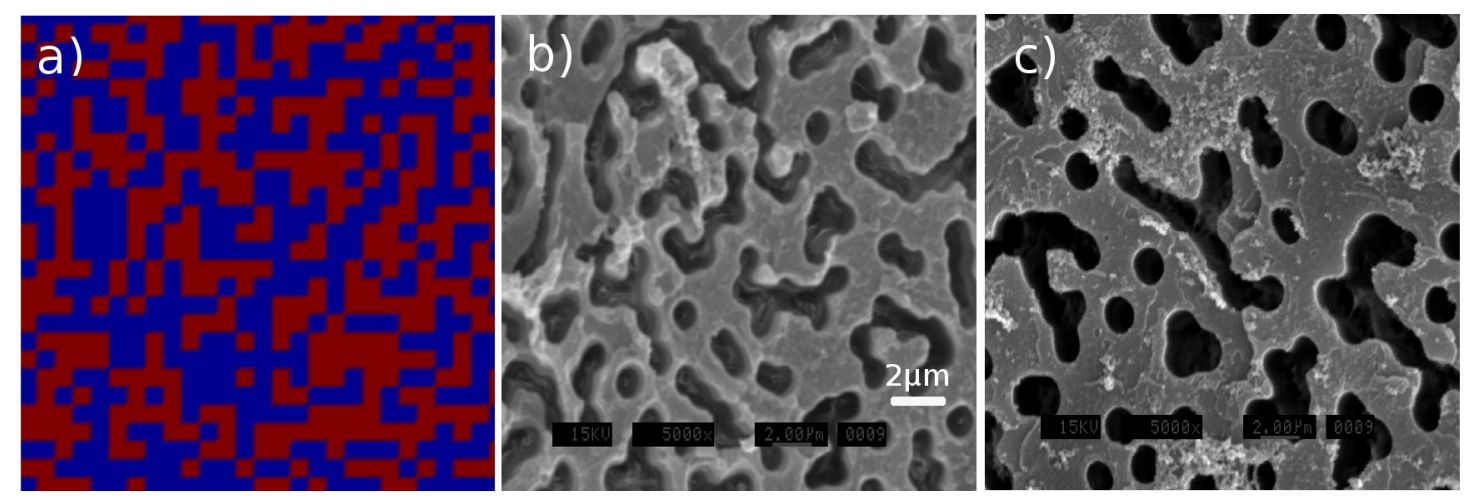

Figure 5.1: (a) An example cross section of a fiber with site size of $0.9 \mu \mathrm{m}$ used for our simulations, corresponding to a fiber of side width equal to $250 \mu \mathrm{m}$. (b) and (c) SEM images of the fibers with the side width of $150 \mu \mathrm{m}$ and $250 \mu \mathrm{m}$, respectively. All three figures (a), (b), and (c) only feature a $24 \mu \mathrm{m} \times 24 \mu \mathrm{m}$ region of the total fiber cross section for a more clear view. The captions on the SEM images show $15 \mathrm{KV}$ at $5000 \mathrm{x}$ magnification, with a marker to show the physical scale of the images. The darker regions in the SEM images indicate the PMMA material.

our set up. We used an X-ACTO curved carving blade heated to approximately 65 degrees Celsius and the fiber is heated to approximately 37 degrees Celsius. The fiber is directly cleaved by hand on a cutting surface and polished using $0.3 \mu \mathrm{m}$ aluminum oxide lapping sheet (LFG03P from Thorlabs), and inspected under a microscope for the final surface quality check.

In order to simulate the propagation of light in the fiber and observe transverse Anderson localization, a monochromatic Gaussian beam of radius $w$ is launched into the center of the random fiber at $z=0$ and propagated along the fiber by numerically solving the wave propagation equation Eq. 5.1, using the finite difference beam propagation method (FD-BPM) [15,26].

$$
i \frac{\partial A}{\partial z}+\frac{1}{2 n_{0} k_{0}}\left[\nabla_{T}^{2} A+k_{0}^{2}\left(n^{2}-n_{0}^{2}\right) A\right]=0
$$

Eq. 5.1 is the paraxial approximation to the Helmholtz equation, where $A(\mathbf{r})$ is the slowly-varying envelope of the primarily transverse electric field $E(\mathbf{r}, t)=\operatorname{Re}\left[A(\mathbf{r}) e^{i\left(n_{0} k_{0} z-\omega t\right)}\right]$ 
centered around frequency $\omega$ and $k_{0}=2 \pi / \lambda . n(x, y)$ is the (random) refractive index of the optical fiber which is a function of the transverse coordinates, while $n_{0}$ is average refractive index of the fiber. The forward propagation scheme is implemented using the fourth order Runge-Kutta method [18]. In order to obtain converged, stable, and non-dissipative simulations, we adopted $\delta \leq \lambda$ and $\delta \leq d$, where $\delta$ is size of the transverse grid in the finite-difference numerical scheme.

The size of the steps in the longitudinal direction are picked as $d z=\alpha n_{0} k_{0} \delta^{2}$. $\alpha=1 / \sqrt{2}$ is the standard stability condition for the Runge-Kutta method in a uniform medium. For our simulations we choose $\alpha=0.02$, in order to guarantee stability and also ensure no power dissipation for reliable long distance propagation. Transparent boundary condition [29] is implemented to absorb the wave that hits the lateral boundaries. In order to properly observe the localization effect, the size of the simulation domain should be selected to be considerably larger than the localization length in each case; therefore, the total power in the simulation region will remain unchanged along the fiber for Anderson localized beams. However, as we will discuss later, localization is still possible even if the tail of the optical field reaches the boundaries of the domain; we will show that the sharp index contrast at the fiber-jacket interface can assist the localization, though it can no longer be strictly viewed as Anderson localization.

We also note that we compared the results of the scalar wave equation with the full vectorial beam propagation method and the localization radius calculations were in excellent agreement. In order to increase the numerical efficiency of our large-scale simulations, we chose to use the scalar wave approximation for all the results obtained here. 


\subsection{Impact of the site size on localization radius}

We use $\xi$ as a measure of the effective beam radius (localization length, when the beam reaches its localization length), given by the variance method [13] as

$$
\xi(z)=\sqrt{\left\langle A(\mathbf{r})\left|(\mathbf{R}-\overline{\mathbf{R}})^{2}\right| A(\mathbf{r})\right\rangle}
$$

where the angle brackets denote integration over transverse $x-y$ coordinates. $\mathbf{R}=$ $(x, y)$ is the transverse position vector and $\overline{\mathbf{R}}$ is the vector pointing to the center of the beam, defined as the mean intensity position by $\overline{\mathbf{R}}=\langle A(\mathbf{r})|\bar{R}| A(\mathbf{r})\rangle$. The optical field is assumed to be normalized according to $\langle A(\mathbf{r}) \mid A(\mathbf{r})\rangle=1 / 2$. We already noted that we carried out our measurements according to the same procedure described in our recent work [20]. We carried out our simulations with the physical parameters related to the experiment described above: $p=50 \%, n_{1}=1.49$, and $n_{2}=1.59$.

In Fig. 5.2, we plot $\xi(z)$ as a function of the propagation distance along the fiber with different values of the site size: $d=0.9 \mu m$ corresponding to a fiber side width of $250 \mu \mathrm{m}$, and $d=0.6 \mu \mathrm{m}$ corresponding to a fiber side width of $150 \mu \mathrm{m}$. The green band represents our experimental measurements of the effective beam radius over an ensemble of 100 separate measurements for $250 \mu \mathrm{m}$ wide fibers, where the green-

highlighted region corresponds to $\xi_{\text {avg }} \pm \sigma_{\xi}$. $\xi_{\text {avg }}$ is the average of the measurements and $\sigma_{\xi}$ corresponds to the standard deviation. Similarly, the red band corresponds to 100 separate measurements for $150 \mu m$ wide fibers, where the overlap between red and green region is only $\sim 2-3 \mu m$ in Fig. 5.2.

In order to collect the 100 different measurements to carry out the required statistics, we used 20 different pieces of disordered fiber with the length of $5.5 \mathrm{~cm}$. We ensured that the fiber side width remains invariant along each sample, by inspecting each fiber 


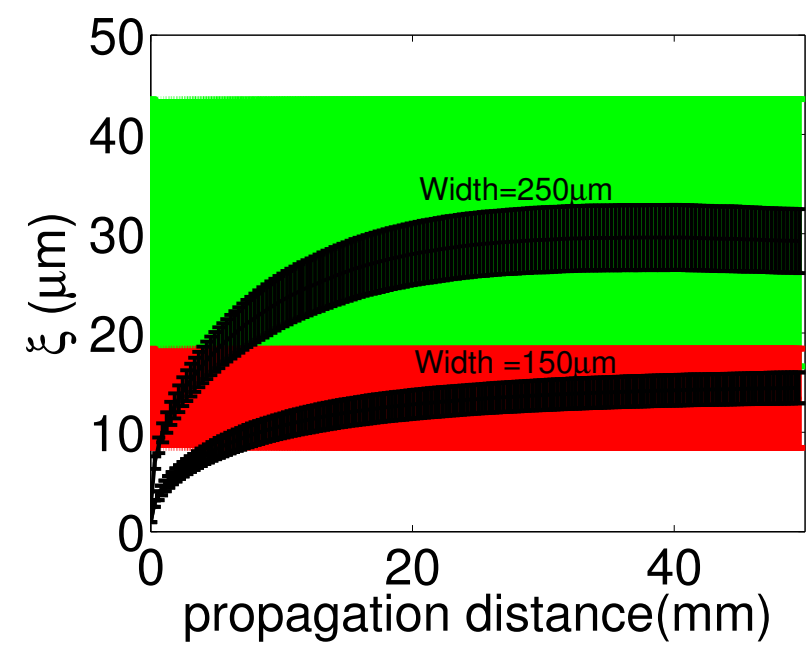

Figure 5.2: Effective beam radius versus propagation distance for different values of the site size: $d=0.9 \mu \mathrm{m}$ corresponding to a fiber side width of $250 \mu \mathrm{m}$, and $d=0.6 \mu \mathrm{m}$ corresponding to a fiber side width of $150 \mu \mathrm{m}$. The mean beam radius in the case of the fibers with side width of $150 \mu \mathrm{m}$ is greatly affected by the large refractive index step at the boundary of the fiber, otherwise it would have been even larger than that of the fibers with side width of $250 \mu \mathrm{m}$. We note that the one standard-deviation regions for experimental measurements marked with green and red color are from the measurements at the end of the fiber samples at the $5.5 \mathrm{~cm}$ length.

under an optical microscope. For each sample, we moved the incident beam to four different points around a best localized spot with the separation of $10 \mu \mathrm{m}$ on a square grid; therefore, 5 different launch positions were explored for each sample, resulting in a total of 100 separate measurements. We should point out that for each sample, the near-field intensity at the output clearly followed the shift in the location of the incident field. The near filed profile for each case was recorded using a 40x objective and a CCD camera.

The results of our simulations are averaged on an ensemble of 100 separate simulations, where the profile of each element of the ensemble is randomized differently, corresponding to a fixed fill-fraction of $p=50 \%$. We use different seeds in our random generator to generate the 100 different random profiles, while keeping the incident beam fixed at the center of the disordered fiber. For each set of simulations, the re- 
gion corresponding to $\xi_{\text {avg }} \pm \sigma_{\xi}$ is highlighted in black, where the average localization length $\xi_{\text {avg }}$ and standard deviation $\sigma_{\xi}$ are calculated over the 100-element ensemble for the field profile after $5 \mathrm{~cm}$ of propagation. Therefore, the error bars signify the expected variation in the effective beam width among the elements of the "statistically" identical ensemble. The input Gaussian beam initially undergoes diffusive broadening $[13,15]$, until its effective radius becomes of the order of the localization length, after which further expansion is halted and the beam remains localized. The mean effective beam radius for the 100 measured near-field beam intensity profiles for the $250 \mu \mathrm{m}$ wide fibers was calculated to be $\xi_{\text {avg }}=30.1 \mu \mathrm{m}$, with a standard deviation
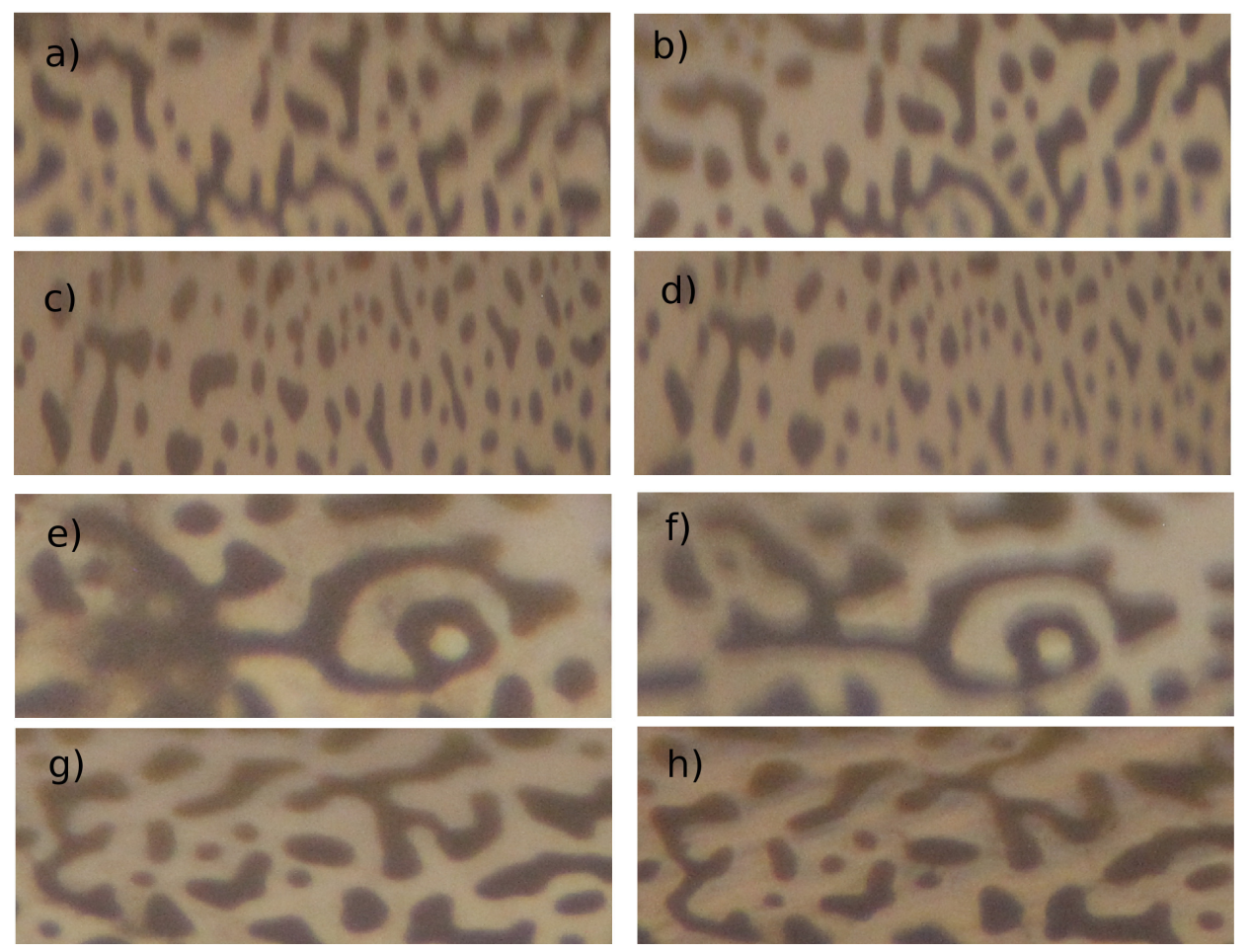

Figure 5.3: (a) and (b) show the refractive index profile of a sample optical fiber with $1 \mathrm{~mm}$ side width taken at different locations along the fiber $(5 \mathrm{~cm}$ apart). The images are taken with an optical microscope and are zoomed in at a small region on the cross section of the fiber and clearly show that the refractive index profile remains invariant along the fiber over the $5 \mathrm{~cm}$ long samples. Similarly, the (c) - (d) pair, (e) - (f) pair, and (g) - (h) pair are taken each at $5 \mathrm{~cm}$ apart locations along the fibers where the optical microscope is zoomed in over the same regions for each pair but different regions for different pairs across the fiber tip. 
$\sigma_{\xi}=13.5 \mu \mathrm{m}$; the numerical simulation resulted in $\xi_{\text {avg }}=29.3 \mu \mathrm{m}$ and $\sigma_{\xi}=3.3 \mu \mathrm{m}$. For the $150 \mu \mathrm{m}$ wide fibers, we obtained $\xi_{\text {avg }}=13.4 \mu \mathrm{m}$ and $\sigma_{\xi}=5.0 \mu \mathrm{m}$ from the experimental measurements, and $\xi_{\text {avg }}=14.2 \mu \mathrm{m}$ and $\sigma_{\xi}=1.7 \mu \mathrm{m}$ from the numerical simulations, respectively. The initial beam radius for our numerical simulations was $2.4 \mu \mathrm{m}$, and for our experimental measurements was $2 \pm 0.25 \mu \mathrm{m}$; the experimental value was dictated by the mode field radius of the Thorlabs SMF 630hp fiber used to launch the beam into the disordered fibers.

The simulations are in good agreement with the experimental measurements, yet the variation in the experimental measurements of the beam radius is larger than the variation in the theoretical results. The difference can be attributed to the variations in the side width of the optical fiber in the draw process that result in approximately $20 \%$ sample-to-sample variation in the side width of the 20 samples used in our measurements. We should note that even though we have ensured that the side width of our samples do not change along the $5.5 \mathrm{~cm}$ length, the samples are cut from a 10-meter long segment of the fiber with an average side width of $250 \mu \mathrm{m}$ (or $150 \mu \mathrm{m}$ for the samples with the smaller side width), with an approximate variation of about 20\%. For our simulations, we used the average value of the side width $(250 \mu \mathrm{m}$ or $150 \mu \mathrm{m})$. We expect to observe the same level of variation in the site sizes for each fiber $(0.6 \pm 0.12 \mu \mathrm{m}$ for the $150 \mu \mathrm{m}$ samples and $0.9 \pm 0.18 \mu \mathrm{m})$ for the $250 \mu \mathrm{m}$ fiber samples, because of the conservation of mass in the draw process. This fact can also explain the $\sim 2-3 \mu \mathrm{m}$ overlap of the localization radius in the experimental data for the two different side widths in disordered fibers, as reported in Fig. 5.2.

In order to show that the refractive index profile remains invariant along the fiber, we have taken images of the index profile across the tip of a sample fiber with 1 $\mathrm{mm}$ side width using an optical microscope at two different locations along the fiber, which are $5 \mathrm{~cm}$ apart. The optical microscope is zoomed in at 4 different locations 
across the fiber at $z=0 \mathrm{~cm}$ where Figs. 5.3(a), 3(c), 3(e), and 3(g) are obtained; these images must be compared with Figs. 5.3(b), 3(d), 3(f), and 3(h), respectively, which are taken at $z=5 \mathrm{~cm}$. Therefore, the pair of Figs. 5.3(a) and 3(b) correspond to the same (zoomed-in) location across the fiber, but $5 \mathrm{~cm}$ apart along the fiber; they clearly show that the refractive index profile remains invariant along the fiber over the $5 \mathrm{~cm}$ long sample. Similarly, the Figs. 5.3(c) and 3(d) pair, Figs. 5.3(e) and 3(f) pair, and Figs. 5.3(g) and 3(h) pair correspond to the same (zoomed-in) location across the fiber (different location among different pairs), but $5 \mathrm{~cm}$ apart along the fiber. We note that finding the matching regions is a rather laborious task due to the difficulty in obtaining consistent high quality optical images of the tip of the fiber; the quality of the images are generally limited by the difficulties in the preparation quality of the fiber cleaves as explained in our recent publication [20]. We also note that our choice of the $1 \mathrm{~mm}$ side width fiber sample instead of the $250 \mu \mathrm{m}$ or $150 \mu \mathrm{m}$ fiber samples was both dictated by the higher quality of the cleave in the $1 \mathrm{~mm}$ sample without having to polish the fiber (polishing lowers the quality of the optical images), as well as the higher image resolutions; we expect that the profiles do not change when the fiber is drawn further down to $250 \mu m$ or $150 \mu m$ side width.

In Fig. 5.2, we might develop the impression that the localized beam radius is smaller for the optical fibers with smaller values of the site size (side width of $150 \mu \mathrm{m}$ ), which is also well supported by the experimental measurements. However, our detailed numerical analysis revealed that the boundary of the fiber in our experiments is playing an important role in setting the observed localization radius. The tails of optical field in the simulation window for the fiber with the $150 \mu m$ side width reach the boundary after a few millimeters of propagation. If a large step index contrast is used at the boundary to confine the field, similar to the experimental conditions where the fiber jacket is "air", a confinement is observed which in every aspect resembles the 
standard Anderson localization, including the exponential decaying tails of the field. Moreover, the simulations agree closely with the localization radius measured over the 100 samples. Considering the fact that two-dimensional systems are always localized,

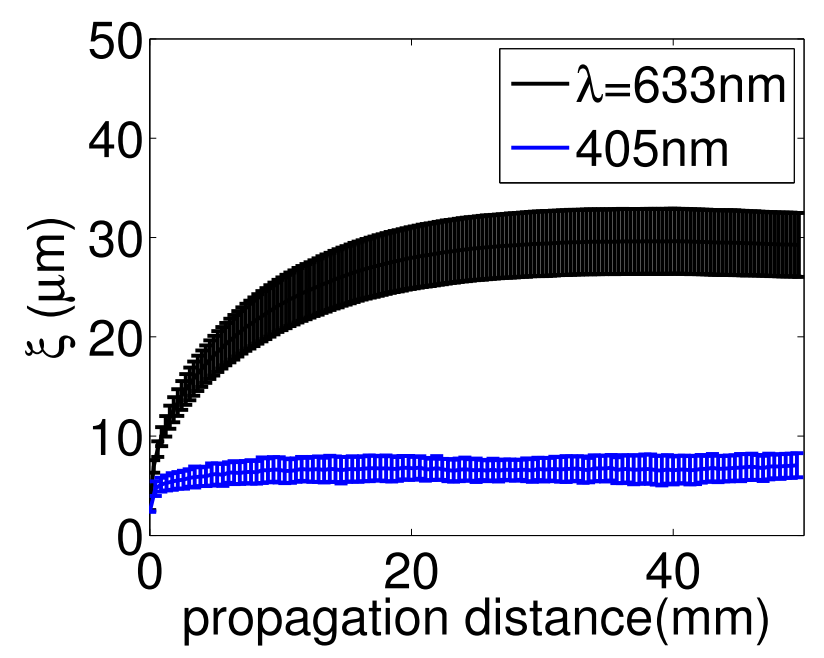

Figure 5.4: The effect of reducing the incident wavelength $(\lambda)$ on the localization $\operatorname{radius}(\xi)$ versus propagation distance.

we extended the simulation window to observe the true localization radius of this geometry (without the impact of the boundary), and observed that it is substantially larger than what we present in Fig. 5.2. In fact, in the absence of the boundary step index, when the fiber side width is taken to be much larger with the same small site size of the $150 \mu m$ fiber, the localization radius is around $50 \mu m$, which is even larger than that of the fiber with the larger values of site size (side width of $250 \mu \mathrm{m}$ ). The impact of the boundary on the localization radius is very important where the signatures (such as the exponential decay of the tails of the localized field) remain similar to those of the ordinary Anderson localization; such an impact has already been explored in a similar context in finite 1D and 2D disordered lattices in Refs. [33, 51].

Szameit et al. [33] have shown that the localization effect is reduced near the truncated boundary of a 1D lattice and in order to maintain the same degree of localization, a higher level of disorder is required near the boundary. Same way, Jovic et al. [51] 
explore the boundary effect in 2D disordered lattices and draw similar conclusions to those of Ref. [33]. Our observations are consistent with and somewhat complement those reported by Refs. [33,51]. We observe the impact of the boundary on the localization radius even when the beam is launched at the center of the fiber, away from the boundary. The key point is that the beam for the specific case of the $150 \mu \mathrm{m}$ fiber with the small approximate site size of $0.6 \mu \mathrm{m}$ reaches the boundary during the diffusive expansion stage (before reaching the localization stage) and the reflections from the boundary affect its final stabilized radius, yet its profile resembles that of the Anderson localized beam.

We emphasize again that for the case of the $250 \mu \mathrm{m}$ fiber with the approximate site size of $0.9 \mu \mathrm{m}$, the choice of boundary condition (absorbing boundary condition versus an air jacket) makes virtually no difference in our results, because the beam that is launched in the center only excites the localized modes that are not impacted by the boundary. However, for the $150 \mu \mathrm{m}$ fiber with the small approximate site size of $0.6 \mu \mathrm{m}$, if we do not consider an air jacket in our simulations (and use absorbing boundary condition instead), we observe the power absorption of about $40 \%$, after 5 cm of propagation; therefore, the presence of air jacket is required to get a consistent result that compares with the experimental measurements.

In Fig. 5.4, the localization radius in the polymer disordered fiber with the side width of $250 \mu \mathrm{m}(0.9 \mu \mathrm{m}$ site sizes) at the incident wavelength of $405 \mathrm{~nm}$ is compared with the one at the wavelength of $632.8 \mathrm{~nm}$. Our calculations show the shorter incident wavelength results in a smaller localized beam radius. This is expected, considering the fact that Maxwell's equations are scale invariant and a shorter incident wavelength is equivalent to larger site sizes, which also results in a smaller localized beam radius. For the wavelength of $1550 \mathrm{~nm}$ which is interesting for telecommunication applications, the localized beam radius is so large that cannot properly localized within the boundaries 
of our fiber with the side width of $250 \mu \mathrm{m}$. In order to observe localization at the wavelength of $1550 \mathrm{~nm}$, the side width of the fiber should be expanded to beyond $500 \mu \mathrm{m}$.

In order to further confirm that our observations are in agreement with the standard signature of the Anderson localization, i.e. exponential decay of the tails of the field in the presence of disorder, we plot a cross section of the intensity profile of the localized beam, averaged over 100 samples in dB units. Fig. 5.5(a) shows a comparison between the experimental results for fibers with side width of $150 \mu m$ and $250 \mu m$. Fig. 5.5(b) shows a comparison between the experimental and numerical results for fibers with side width of $150 \mu \mathrm{m}$, where the difference between simulation and experiment is caused by the larger variation in the experimental results (see Fig. 5.2), and also the noise in the CCD beam profiler at low intensities. Similar results for the $250 \mu \mathrm{m}$ fiber were already reported in our recent publication [20].

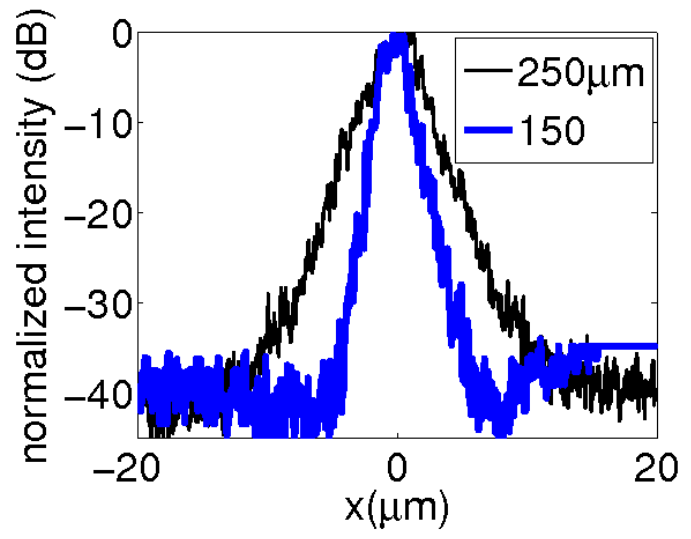

a)

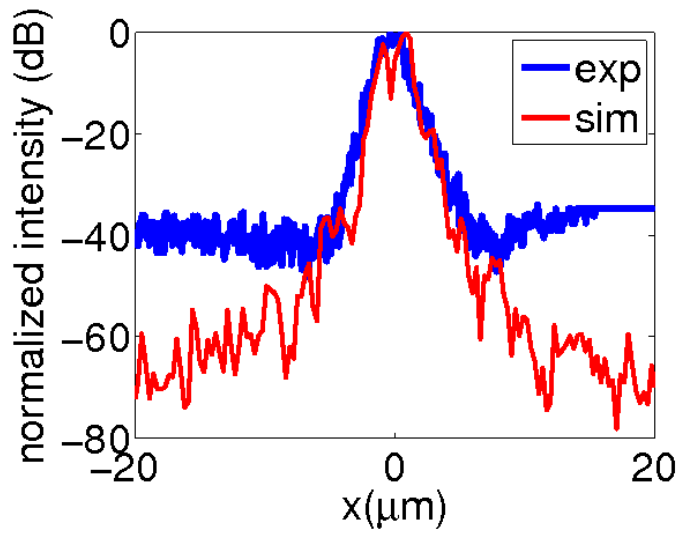

b)

Figure 5.5: Cross section of the intensity profile of the localized beam averaged over 100 samples of raw data in $\mathrm{dB}$ units. (a) shows a comparison between the experimental results for fibers with side width of $150 \mu \mathrm{m}$ and $250 \mu \mathrm{m}$. (b) shows a comparison between the experimental and numerical results for fibers with side width of $150 \mu \mathrm{m}$, where the difference between simulation and experiment is caused by the larger variation in the experimental results (see Fig. 5.2), and also the noise in the CCD beam profiler at low intensities. 


\subsection{Impact of the fill-fraction on the localization ra- dius}

Our choice of the fill-fraction parameter $p=50 \%$ is optimal, in order to obtain the minimum localization length in these fibers. In order to examine the effect of the fill-fraction $p$ on the localization length, we numerically study the case of $p=40 \%$ and compare the results with $p=50 \%$ which was outlined in the previous section. In Fig. 5.6, we show the results of our simulations, where we plot $\xi(z)$ as a function of the propagation distance along the fiber. Each curve, relating to a different value of the

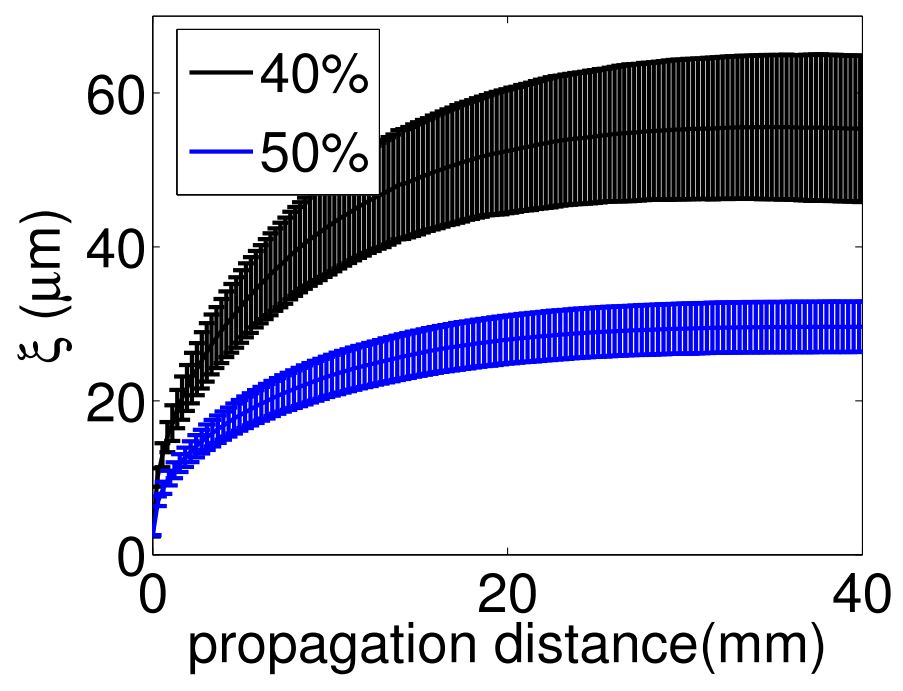

Figure 5.6: Effective beam radius versus propagation distance for different values of the fillfraction of $p=40 \%$, and $p=50 \%$. The optimal fill-fraction of $p=50 \%$ results in the lowest effective beam radius and localization length.

fill-fraction parameter $p$, represents the average of 100 independent simulations. The error bars indicate the standard-deviation of $\xi(z)$ calculated over each 100-element ensemble. The localization radius for a given value of $p$ always fluctuates around an average value because each realization of disordered fiber has a different refractive index profile. From Fig. 5.6, it is clear that the lower localization length is obtained 
for $p=50 \%$. Any decrease in the value of the fill-fraction from the optimal choice of $p=50 \%$ results in an increase in the localization length. This observation agrees well with the physical intuition that $p=50 \%$ increases the overall probability of scattering and should result in a smaller localization length. We note that in order to obtain localization at smaller values of $p$, we needed to increase the side width of the simulation area in order to prevent the scattered light to reach the absorbing boundaries (250 $\mu \mathrm{m}$ for $p=50 \%$ and $300 \mu m$ for $p=40 \%$ ), and for lower fillfractions, the domain size needs to increase accordingly. While we verified this for a few samples, we decided against carrying out a full scale analysis for smaller values of $p$, due to the huge computational cost, which would have amounted on months of simulations on a large cluster. However, as shown in section 5.6, we carried out full simulations for $p=30 \%$, as well, because the required simulation window was smaller due to the larger values of index contrast, resulting in smaller localization radius. As we discussed before, above the percolation threshold ( $p>59.27 \%)$, index-guiding by the higher-index localized clusters can mask any effects that can be attributed to Anderson localization. In practice, we have observed that for the choice of parameters used for our simulations, the effective localized beam radius increases monotonically with increasing value of the fill-fraction $p$ beyond the optimal value of $p=50 \%$.

In the presence of large disorder, for example at $p=50 \%$, little variation is expected among the individual elements of the ensemble due to a self-averaging behavior, which was also pointed out in our recent publication [20]. This "self-averaging" behavior is generally obtained from the theory of wave localization under appropriate conditions (see for example Refs. $[38,39]$ ) and is responsible for the small size of the error bars at $p=50 \%$. However, when the amount of disorder is low, such as for small values of $p$ in our work or for small refractive index contrast in the case of Ref. [15], self-averaging may not hold and wave localization is only meaningful in a statistical averaging sense. 


\subsection{Impact of refractive index contrast}

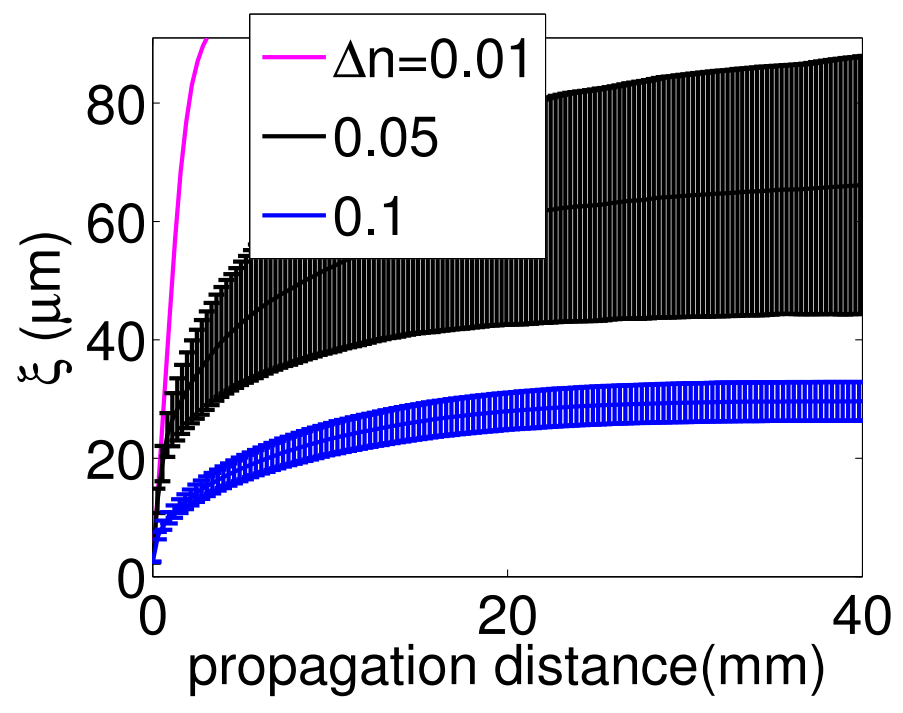

Figure 5.7: Effective beam radius vs propagation distance for different values of refractive index contrast $\Delta n$. Larger index contrast results in smaller localization radius.

In this section, we show that lowering the index contrast $\Delta n=\left(n_{1}-n_{2}\right)$ increases the localization length. If the index contrast is too low, the beam of light will expand until it reaches the edges of the sample and transverse localization may never materialize in practice. This can be observed in Fig. 5.7, where the effective beam radius for $p=50 \%$ and different values of refractive index contrast $\Delta n$ are shown. Our simulations clearly show that for a waveguide with the side dimension of $\approx 400 \lambda(250 \mu \mathrm{m}$ at $\lambda=632.8 \mathrm{~nm})$, the wave does not get localized for the refractive index contrast of 0.01 , i.e. the localization length is larger than the side dimension of the structure, so the light is absorbed by the absorbing boundaries. We remind that two-dimensional random systems are always localized, however, the localization length can be larger than the domain of interest, such as discussed above. In order to observe the localization effect for the low refractive index contrast of 0.05 , we need to use a larger side dimension for the waveguide of approximately $350 \mu m(555 \lambda)$. We note that for all the simulations in this section, we only used absorbing boundary condition; therefore, the boundary 
did not impact the localization condition.

\subsection{Glass Anderson localized optical fibers}

In order to use Anderson localized optical fibers for possible applications in the telecommunication wavelengths, we need to have a disordered fiber with low loss materials at the telecommunication window of the spectrum: therefore, glass fibers are more desirable than polymer fibers. Here we numerically investigate the glass Anderson localized optical fibers with random air voids. As far as our numerical simulations are concerned, everything remains the same, except the index contrast, which is now substantially larger, resulting in a smaller localization radius, which is more desirable. The refractive index profile is implemented based on the same procedure described for polymer optical fiber, yet the refractive index of sites are randomly picked as $n_{2}=1.5$ and $n_{1}=1.0$ for the glass host and random air voids, respectively. The side width of each disordered fiber is $100 \mu \mathrm{m}$ and site sizes are $0.6 \mu \mathrm{m}$. We note that for the glass-air structure with the refractive index contrast of 0.5 , the fiber dimensions can be chosen to be smaller compared with that of the polymer fiber, because the localization radius is smaller.

In Fig. 5.8, the calculated beam radius $\xi(\mu m)$ versus propagation distance for different values of fill-fraction are plotted, where each simulation is again performed for 100 different realization of randomness (100 different refractive index profiles). Similar to the case of the disordered polymer fiber, we observe that the localization radius is lowest for the optimal fill-fraction of $p=50 \%$. We also observe that the beam radius of the localized beam for the glass Anderson localized optical fibers with random air voids is smaller than the beam radius in the polymer fibers, because of the larger index contrast. These observations are consistent with our results in the previous section 


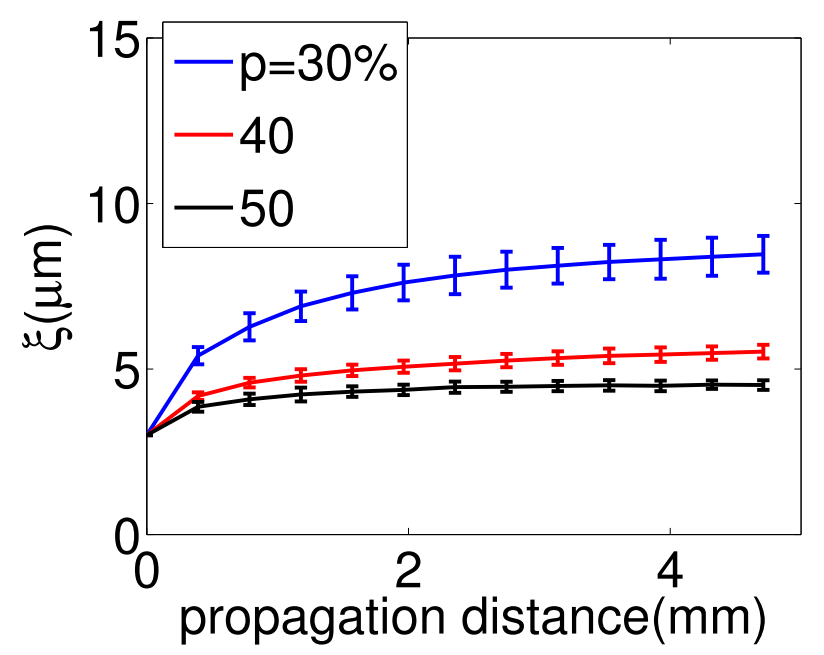

Figure 5.8: Effective beam radius vs propagation distance for different values of fill-fraction, $\mathrm{p}$, in glass disordered optical fibers with random air voids.

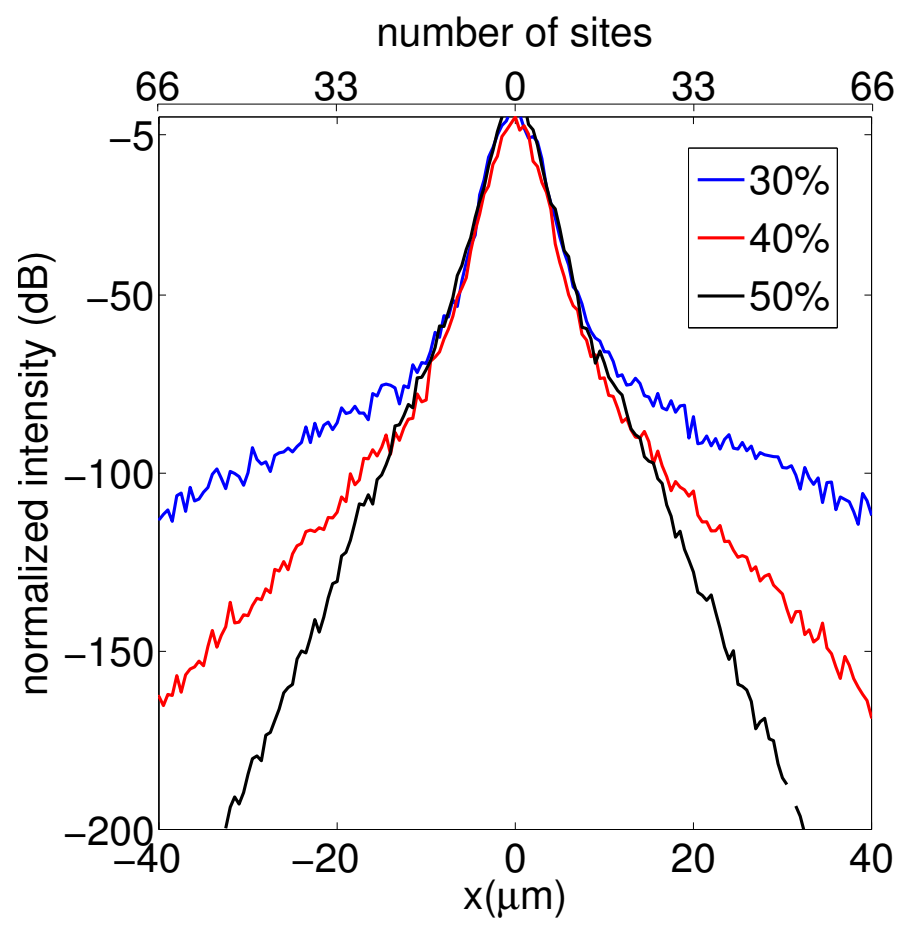

Figure 5.9: Exponential decay of the average intensity for different values of fill-fractions, $p$, in glass disordered optical fibers with random air voids. 
on the impact of the refractive index contrast on the localization radius. Another important advantage of the glass disordered optical fibers is that the larger index contrast results in a stronger self-averaging behavior, reducing the standard-deviation in the value of the beam radius; therefore, glass Anderson localized optical fibers provide a more reliable and predictable behavior for potential applications in optical fiber communications.

The exponential decay of the tail of averaged intensity for different values of fillfraction are shown in Fig. 5.9, which presents a clear proof of Anderson localization in each case. As the exponential tails show, for lower values of fill-fraction, the decay coefficient is smaller and the wave expands farther. In the case of optimal localization for $p=50 \%$, the decay coefficient is the largest and the wave gets localized much faster. It must be noted that the region with an appreciable intensity difference between the $p=50 \%$ and $p=30 \%$ structures has lower intensity than the peak value by at least $70 \mathrm{~dB}$; therefore, it will be extremely hard to distinguish between the beam profiles of the two structures, using conventional experimental techniques.

\subsection{Conclusions}

We explored the effect of site size, fill-fraction, and refractive index contrast for polymer optical fibers. We showed both numerically and experimentally that the large refractive index step at boundary of the fiber results in an anomalous reduction in the value of the localized beam radius, when the site sizes are decreased; this observation is consistent with and somewhat complement those reported by Refs. [33,51]. Our results show that the boundaries of the fiber assist the wave localization of the weakly localized modes, even though the incident beam is at the center of the fiber and away from boundaries. We also showed that $p=50 \%$ is the optimum fill-fraction to have the 
lowest value for the localized beam radius and also the minimal impact of boundaries on the wave localization. Lowering the refractive index contrast of the materials from which the fiber is drawn results in the increase of the localized beam radius. Using numerical simulations, we observed that a glass host with disordered air voids results in a substantially reduced value of the localized beam radius. The impact of other parameters in glass disordered fibers were similar to those of the polymer disordered fibers.

We would also like to point out that a typical reliable simulation of transverse Anderson localization for our optical fibers requires a transverse area in the range of $\sim 10^{5}-$ $10^{6} \lambda^{2}$, and $\sim 10^{6}-10^{7}$ steps in the longitudinal direction, which is computationally intensive. Therefore, an ensemble of 100 disordered fiber simulations to obtain proper statistics requires approximately $10^{5} \mathrm{CPU}$ hours. The simulations were carried out on a local large HPC cluster consisting of 142 Nehalem 5550 nodes (1,136 cores), with 24 gigabytes of memory per node and a high-throughput, low-latency Infiniband network. As yet another evidence of the strong transverse Anderson localization, we scan the in-coupling single-mode fiber across the input facet of the our disordered fiber, and image the near-field output from the disordered fiber on a CCD camera, using a 40x objective. 


\section{Chapter 6}

\section{Transverse Anderson localization in a glass optical fiber with disordered air voids}

\subsection{Introduction}

Anderson localization [1], especially for electromagnetic waves [3], has been of great research interest over the past few years. The observation of strong localization in three dimensional (3D) optical media is quite challenging [6], because optical materials generally cannot provide sufficient scattering strength to satisfy the Ioffe-Regel condition [7]. Wiersma, et al., [11] reported the first experimental evidence of Anderson localization in strongly scattering GaAs powder. The required conditions for localization are considerably relaxed in one dimensional (1D) and two dimensional (2D) systems; in fact, 1D and 2D disordered systems are always localized [2]. In practice, a small localization length, $\xi$, is easily achievable in 1D and 2D systems, even 
for a moderate amount of disorder. For example, De Raedt, et al., [13] have shown that if the randomness in the refractive index profile is only limited to the transverse $\mathrm{x}-\mathrm{y}$ plane, a beam propagating in the z-direction can remain trapped in the transverse direction; a reasonably small localized beam diameter is possible even for small disorder refractive index contrast on the order of $10^{-4}$, as was experimentally observed by Schwartz, et al. [15], in a photo-refractive crystal. Transverse Anderson localization has since been actively explored in various systems, e.g., in 1D disordered photonic lattices [17], in optical waveguide arrays with off-diagonal coupling disorder [32], and in amorphous photonic lattices [52].

We recently reported the first observation of transverse Anderson localization in an optical fiber [20], lacking the conventional core/clad structure, where the large index contrast between constituent polymer materials resulted in an effective propagating beam diameter comparable to that of a typical index-guiding optical fiber. The polymer optical fiber composed of about 80,000 randomly placed sites of polymethyl methacrylate (PMMA) and polystyrene (PS), both of which are commodity polymers, with a refractive index contrast of 0.1 and fill-fraction of $50 \%$. The fill-fraction is the material fraction of the lower index polymer (PMMA, $n=1.49$ ) to the total which also includes the higher index polymer (PS, $n=1.59$ ).

We recently carried out a detailed investigation of the impact of the fiber design parameters on the transverse Anderson localization of light in disordered optical fibers [22]. In general, if transverse Anderson localization is to be used as the waveguiding mechanism in optical fibers, it is desirable to have designs with smaller beam diameters, as well as lower sample-to-sample variation in the value of the beam diameter. The sample-to-sample variation is a natural consequence of the statistical nature of Anderson localization; however, we confirmed that such variations can be suppressed because of "self-averaging" behavior, if the refractive index contrast is increased [22]. In fact, 
we showed that robust designs (small beam diameter and low sample-to-sample variation in the value of the beam diameter) are possible if the index contrast is increased to that of the fused silica $(\sim 1.5)$ to air $(1.0)$.

Here, we present the first results on the fabrication and analysis of a disordered silica glass-air (porous) optical fiber. Although we obtained a fill-fraction that was substantially below the optimal value of $50 \%$, strong localization of light was observed (only) in regions close to the outer boundary of the fiber; i.e., interface near the porous glass and protective polymer coating. This observation is quite interesting, because Szameit, et al., [33] and Jović, et al., [51] have shown that the boundary of a disordered medium has a de-localizing effect and it is easier to observe strong localization in regions away from the boundary. Here, we show that our results do not contradict those of Refs. [33,51], and that the de-localizing effect of the boundary in our fiber is offset by the substantially higher near-boundary air fill-fraction compared with the center of the fiber, which results in a strong transverse scattering and near-boundary localization effect. Our observations also agree with the results recently published in Ref. [53] on the effect of a nonuniform variation in the strength of the disorder in a 1D lattice on the localization of light. They reported that the tail of the localized light decays faster in a more strongly disordered region, and vice versa.

\subsection{Disordered optical fiber with random air voids}

The optical fiber employed in this work was drawn from "satin quartz" (Heraeus Quartz) which is a porous artisan glass. The starting rod had dimensions of $8 \mathrm{~mm}$ in diameter and $850 \mathrm{~mm}$ in length and was drawn at Clemson University on a customdesigned Heathway draw tower at a temperature of $1890^{\circ} \mathrm{C}$. The fiber was coated with a conventional telecommunications single layer UV-cured acrylate coating. 150 
meters of fiber was drawn with an average glass and coated diameter of 250 and $417 \mu \mathrm{m}$, respectively. While it was known that the fill-factor; i.e., degree of porosity, was sub-optimal for the idealized level of localization, the satin-quartz was selected as a convenient and extraordinarily inexpensive expedient.

The cross-section of the fiber was imaged using a Hitachi SU-6600 analytical variablepressure field emission scanning electron microscope (SEM). The fiber was polished to a 1 micron finish and then mounted in the sample holder with carbon tape and placed in the chamber. Images were taken in variable pressure mode using back scattered electron imaging at $20 \mathrm{kV}$.

The cross-sectional SEM image of the disordered porous optical fiber is shown in Fig. 6.1(a), which also provides a good estimate of the refractive index profile of the fiber; the light gray background matrix is glass and the black random dots represent the air voids. The diameter of the fiber is confirmed to be about $250 \mu \mathrm{m}$ and the average air fill-fraction is about $5.5 \%$ with the air void diameters varying between about $0.2 \mu \mathrm{m}$ to $5.5 \mu \mathrm{m}$. The distribution of porosity seems to be consistent along the length of the fiber, likely due to the equally consistent distribution of pores along the length of the rod as is what gives "satin quartz" its opalescent qualities.

To observe the transverse Anderson localization effect, we use the light from a $405 \mathrm{~nm}$ diode laser delivered using a 630hp fiber from Thorlabs, where the average mode field diameter of this slightly-multimode fiber is around $4 \mu \mathrm{m}$. The $630 \mathrm{hp}$ fiber is buttcoupled to the disordered fiber and the output beam profile is measured using a $40 \mathrm{x}$ objective on a CCD camera, as explained in detail previously [20,22]. In Ref. [22], we showed that a shorter wavelength results in a stronger localization effect and consequently a smaller localization radius. The disorder fill-fraction in the fiber samples studied here is relatively low. Therefore, we decided to use a $405 \mathrm{~nm}$ diode laser, 

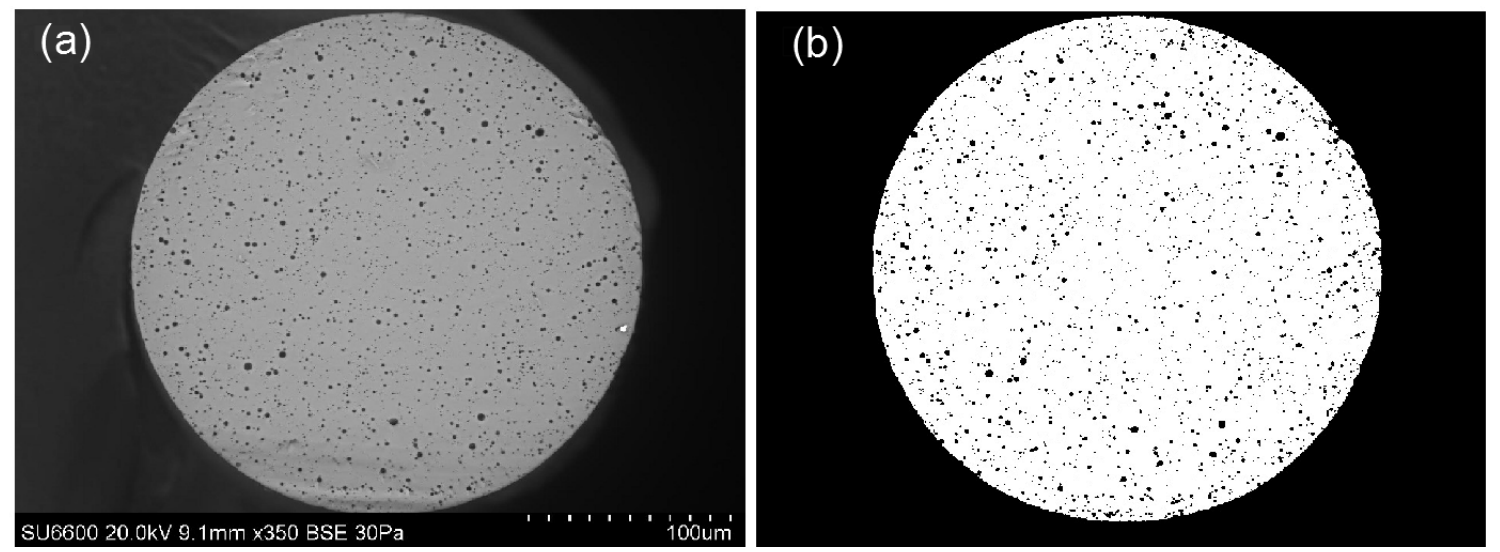

Figure 6.1: (a) SEM image of the glass optical fiber with random air voids. For ease of viewing, the polymer coating has been removed. (b) Refractive index profile used in our simulations.

rather than the $633 \mathrm{~nm}$ He-Ne laser, which was previously used in Ref. [20].

The finite difference beam propagation method (FD-BPM) was used to carry out the simulations [22]. The refractive index profile is extracted from the SEM image of the fiber in Fig. 6.1(a) and is directly used in the FD-BPM program; the refractive index profile used in our simulations is shown in Fig. 6.1(b).

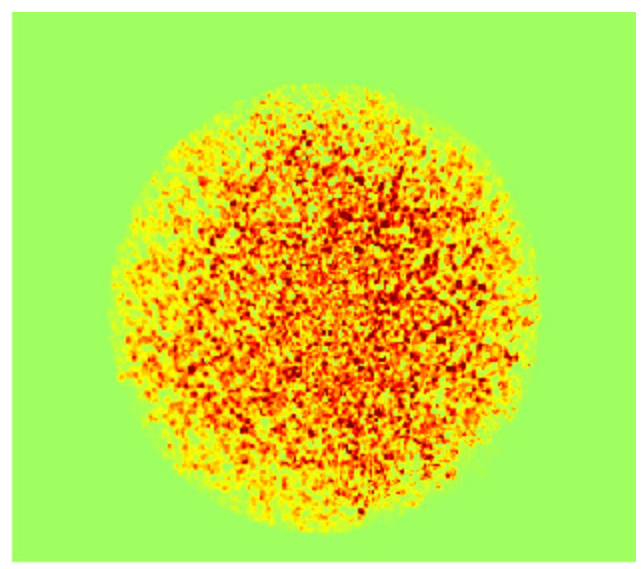

Figure 6.2: The experimental measurement of the near-field intensity when the beam is launched near the center of the fiber, where no localization is observed.

Fig. 6.2 shows a typical result from launching of the beam (405 nm wavelength) into the center of the fiber. It is clear that the disorder is not sufficient to clamp the beam 
radius to a value smaller than the diameter of the fiber; therefore, the beam fills the entire cross section of the fiber. In the sections to follow, we will show that the beam can remain localized, if the light is coupled near the outer boundary of the fiber. We also show that the stronger localization of the beam near the boundary is due to the higher disorder density (air fill-fraction) in the regions near the boundary of the fiber.

\subsection{Localization near the outer boundary}

In order to investigate the localization profile of the beam, the $630 \mathrm{hp}$ fiber was scanned across the input facet of the disordered fiber near its outer boundary region; i.e., porous glass/coating interface. The near-field intensity at the output facet of the disordered fiber is captured and processed to be compared with the theoretical simulations. We selected 10 different fiber samples, each approximately $10 \mathrm{~cm}$ long, and measured the near-field intensity at 10 different locations near the outer boundary of each fiber for a total of 100 independent measurements. The "localized" near-field intensity for four different incident spots near the outer boundary is shown in Fig. 6.3; in each case, the localized spot consists of multiple peaks, which are located near the boundary.

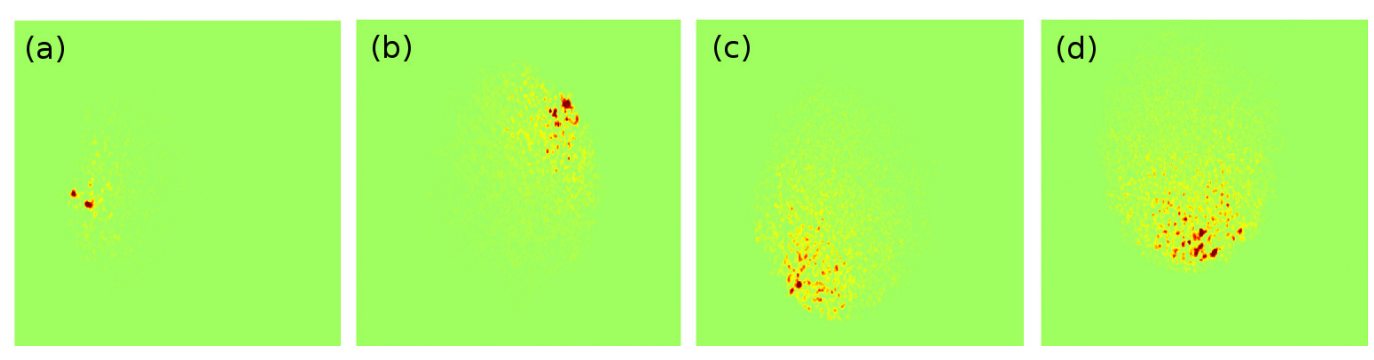

Figure 6.3: Near-field intensity measurements at the output facet of the disordered fiber samples for 4 different launch positions.

The 100 independent measurements should be sufficient to capture the statistical nature of localization and to investigate the exponential decay of the intensity tail. 
We repeated the same procedure outlined above in our simulations, using the refractive index profile shown in Fig.6.1(b), and collected 100 separate near-field intensity profiles, using incident beams launched at different positions near the outer boundary of the fiber. The beam localization for four different incident spots near the outer boundary are shown in Fig. 6.4; again, in each case, the localized spot consists of multiple peaks, which are located near the boundary. Similar to the experimental

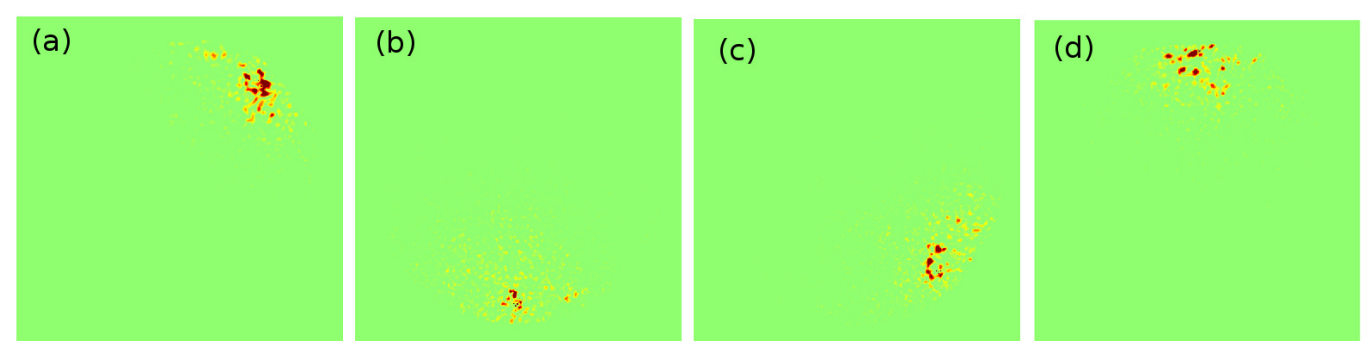

Figure 6.4: Near-field intensity simulations at the output facet of the disordered fiber for 4 different launch positions.

observations, if the beam is launched at the center of the disordered fiber in our simulations, the field fills the entire cross section of the fiber after propagating a distance less than $5 \mathrm{~mm}$.

We calculate the beam localization radius $(\xi)$ using the same method described in Refs. [20,22]. In Fig. 6.5(a), the region highlighted in black corresponds to the theoretical simulation of the effective beam radius $\xi_{\text {avg }} \pm \sigma_{\xi}$ as a function of the propagation distance, where $\xi_{\text {avg }}$ represents the average value of the effective beam radius over the 100 simulated samples (captured at each point along the fiber in the z-direction), and $\sigma_{\xi}$ represents that standard deviation. The effective beam radius expands as the beam propagates along the fiber until it reaches its final localized value, after which the effective beam radius does not change appreciably. For the experimental measurements, we only process the field intensity profiles at the output facet of the fiber; therefore, the region highlighted in red in Fig. 6.5(a) represents the final stabilized mean value and the standard deviation from the measurements, where reasonable agreement is 
observed between theory and experiment. The large values of standard deviations signify the statistical nature of the strong localization effect, and can be considerably lowered for disordered fibers with larger air fill-fraction, as discussed in Ref. [22].

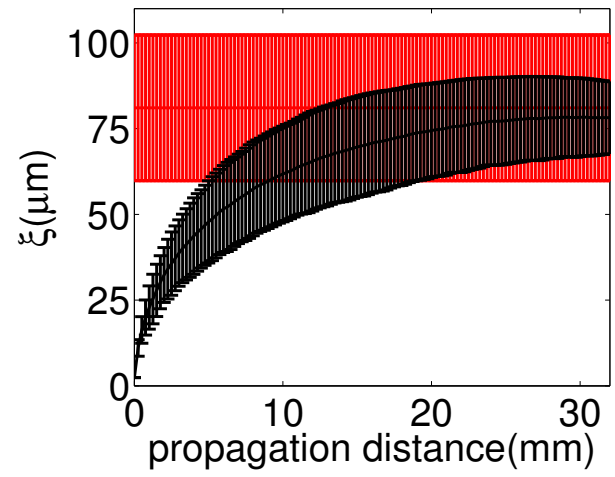

(a)

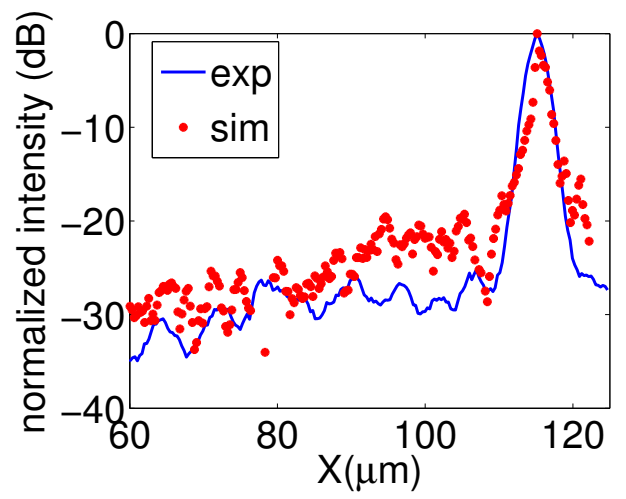

(b)

Figure 6.5: (a) The region highlighted in red corresponds to one standard deviation in each direction around the average experimental measurement of the localization length parameter represented by $\xi_{\text {avg }} \pm \sigma_{\xi}$. The region highlighted in black corresponds to theoretical simulation of the effective beam radius $\xi_{\text {avg }} \pm \sigma_{\xi}$ as a function of propagation distance. (b) Cross section of the intensity profile of the highest peak in the localized beam averaged over 100 samples of raw data from simulations and 100 samples from experiments in dB units.

In order to provide evidence for strong localization of the beams, it is common to show an exponential decay of the tails of the localized intensity profiles, as shown recently [20,22]. However, presenting such a figure in this case is considerably more challenging, because the localized spot is composed of multiple peaks. We observed numerically that the smaller the air fill-fraction, the more separated the peaks are within the localized beam spot, which also results in a larger effective beam diameter. In order to show an exponential decay tail, we selected the highest peak from each sample among the 100 separate measurements. We then averaged the intensity of these highest peaks over the 100 samples, and plotted a cross section of the intensity profile in Fig. 6.5(b) shown as the solid blue line. We repeated the same procedure for the 100 separate numerical simulations and plotted the cross section of the average intensity 
profile of the highest peak in Fig. 6.5(b) marked by the red dots. The experimental and theoretical results are in reasonable agreement. We note that the intensity profile presented in Fig. 6.5(b) is a cross-section in the radial direction; we observed a similar exponential decay behavior in the angular direction. However, this averaging over the highest peak should only be regarded as for illustration purposes, as emphasized earlier. In order to calculate the localization radius, one must include the intensity of all peaks, as is also considered in Fig. 6.5(a).

\subsection{Non-uniform distribution of disorder}

As discussed above, Szameit, et al., [33] and Jović, et al., [51] have shown that the boundary of a disordered medium has a de-localizing effect and that it is easier to observe strong localization in regions away from the boundary. We claim that our observation presented herein of localization "only" near the boundary of the fiber is not contradictory to those of Refs. [33,51] and arise from the nonuniform distribution of disorder across our disordered fiber. In other words, the air fill-fraction is higher near the outer boundary of the fiber, which offsets the de-localizing effect of the boundary and results in a near-boundary strong localization effect. Shown in Fig. 6.6(a) is a density plot of the air fill-fraction (disorder density) over the tip of the disordered fiber; the presence of the larger near-boundary air fill-fraction supports our claim that the higher disorder density offsets the de-localizing effect of the boundary. Moreover, we observe spots around the boundary that have a relatively lower disorder density compared with other near-boundary spots, and these spots are likely responsible for the larger effective localized beam radius observed in intensity profiles such as Fig. 6.3(d) compared with Fig. 6.3(a).

In order to show the (average) radial variation of the disorder density, we average 


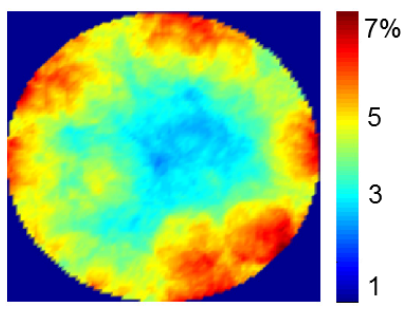

(a)

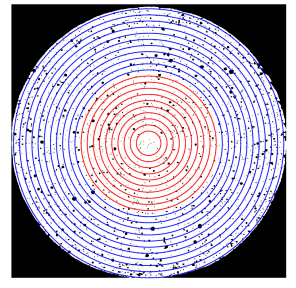

(b)

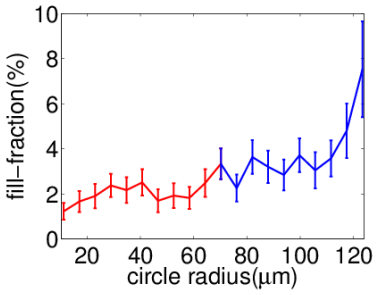

(c)

Figure 6.6: (a) Density plot shows the air fill-fraction over the tip of the fiber, where the disorder level is generally higher near the outer boundary than the central regions. (b) Segmentation of fiber to different regions for averaging over the angular coordinate, where red and blue colors are used in order to make it easier for the reader to distinguish the regions closer to the center versus regions closer to the outer boundary of the fiber. (c) Air fill-fraction averaged over the angular coordinate as a function of the radial coordinate over the tip of the fiber. The error bars signify the change in the value of the air fill-fraction, if the global image threshold varies by 0.07 around an Otsu's threshold of 0.37 .

the air fill-fraction of Fig. 6.6(a) over the angular coordinate and plot the result in Fig. 6.6(c). The actual regions over which the angular averaging is performed are marked with circles in Fig. 6.6(b), where we have used two different colors (blue and red), in order to make it easier for the reader to distinguish the regions closer to the center versus regions closer to the outer boundary of the fiber. We note that in Fig. 6.6(a) and Fig. 6.6(c), we use a global image threshold of 0.37 using Otsu's method [54] to deduce a binary refractive index profile from the SEM image of the fiber end. The error bars in Fig. 6.6(c) show the change in the value of the air fillfraction, if the Otsu's threshold is raised or lowered by 0.07 ; i.e., varying from 0.30 to 0.44. However, we have used the average threshold value of 0.37 in all our simulations. Again, Fig. 6.6(c) clearly shows the increase in the air fill-fraction near the outer boundary, compared with the center of the fiber.

We note that under visual examination, the density of the pores in the original satin quartz preform rod appeared uniform and was likely not responsible for the nonuniform distribution of the disorder across the fiber. We speculate that the nonuniformity was caused by the temperature distribution experienced by the glass during the draw 
process, but further study is warranted to better understand how the porosity and pore distribution changes with processing conditions.

\subsection{Conclusions}

We report the first observation of transverse Anderson localization in a glass optical fiber, where the strong localization happens near the outer boundary, rather than the central region. Previous work by Szameit, et al., [33] and Jović, et al., [51] has clearly shown the de-localizing effect of the boundary in a disordered medium. We have shown that the disorder distribution in our fiber samples is not uniform and we observe a substantially larger air fill-fraction in the regions closer to the boundary of the fiber. Therefore, the higher disorder in regions closer to the boundary offsets the de-localizing effect of the boundary. The air fill-fraction is as low as $2 \%$ in the central regions, but it reaches almost as high as $8 \%$ near the boundary of the fiber.
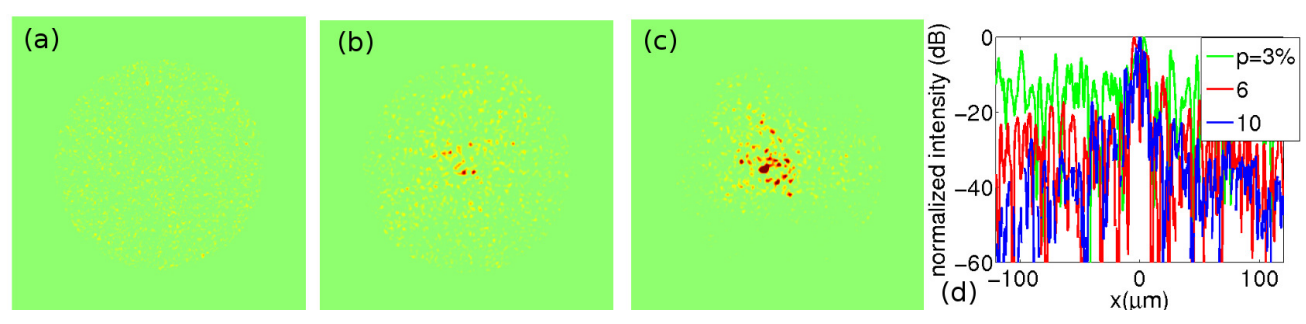

Figure 6.7: Simulation of the near-field intensity profile when the beam is launched near the center of the fiber, for uniform disorder distribution with (a) $3 \%$ air fill-fraction, (b) $6 \%$ air fill-fraction, and (c) $10 \%$ air fill-fraction. (d) Cross section of the intensity profile for uniformly disordered fibers with $3 \%, 6 \%$, and $10 \%$ air fill-fraction, where the beam is launched near the center of the fiber. All figures are plotted for the intensity profile after propagating $5 \mathrm{~cm}$ along the fiber.

In Fig. 6.7, we present our numerical simulations for light localization in the central region of the fiber end at $405 \mathrm{~nm}$ wavelength, when the disorder in the refractive index profile is uniformly distributed, for different values of air fill-fraction. Figs. 6.7(a) and 6.7(d) show that no localization is observed for $3 \%$ uniform air fill-fraction. For 
$6 \%$ uniform air fill-fraction, traces of strong localization can be observed in the central regions as shown in Figs. 6.7(b) and 6.7(d). For 10\% uniform air fill-fraction, strong localization can be clearly observed in the central regions as shown in Figs. 6.7(c) and $6.7(\mathrm{~d})$, and the tails of the field decay considerably faster than the case of $6 \%$ uniform air fill-fraction. Therefore, our results in Fig. 6.7 are consistent with our arguments on why localization can only be observed near the outer boundary of this fiber. We note that the difference between the localization strength in the center of the fiber samples as presented in Fig. 6.7 versus the near-boundary region as explored in Figs. 6.4 and 6.5(b) is very small. Although our results do not contradict those of Szameit, et al., [33] and Jović, et al., [51], the difference in the localization strength falls within the margin of error in our 100-element ensemble used in our simulations. We note that the large standard deviation in the localization radius is the result of the small fill-fraction in the fiber samples, as was also discussed in Refs. [20,22], as well as the large variations in the fill-fraction near the boundary as shown in Fig. 6.6(c). It is possible to conclusively verify the de-localizing effect of the boundary in samples similar to the disordered fibers presented in this work by exploring similar samples with uniform fill-fraction. However, we expect that this analysis would require many more simulations and massive computational resources, which is beyond the scope this paper. Future efforts are focusing on fibers with higher air fill-fraction (50\%) and greater uniformity of the porosity. Potential applications of the disordered optical fibers are in the spatially multiplexed short-haul optical fiber communications, as well as optical imaging. These potential applications will also be explored in the future. 


\section{Chapter 7}

\section{Multi-beam propagation in Anderson localized optical fibers}

\subsection{Introduction}

Multicore optical fibers are an increasingly attractive technology for many applications, such as in optical communications [55-58], sensing [59], optical interconnects [60], optical coherence tomography [61], and imaging [62]. The number and size of waveguiding cores in a multicore fiber depend on the application. Multicore fibers used for imaging or optical interconnects [59-63] can contain hundreds of cores. On the other hand, multicore optical fibers used for optical-fiber communications are limited to a handful of cores, because the crosstalk between the cores is more detrimental than it is in imaging and interconnect applications; the higher number of cores results in smaller core separation and higher crosstalk and degrades communications.

We recently reported on the development of a novel nano-engineered optical fiber [20], which can support the simultaneous propagation of multiple beams with potential 
applications in spatially multiplexed optical-fiber communications and imaging. The beam propagation mechanism in this nano-engineered fiber is based on transverse Anderson localization originally proposed by De Raedt et al. [13], and experimentally observed in various configurations $[15,17,20]$. The refractive index profile of the disordered fiber is invariant in the longitudinal direction; however, the transverse index profile is random. We described the fabrication procedure for a polymer version of an Anderson localized optical fiber (p-ALOF) using polystyrene (PS), $n_{1}=1.59$ and poly(methyl methacrylate) (PMMA), $n_{2}=1.49$ in detail in Ref. [20]. Unlike conventional optical fibers that operate on the index-guiding mechanism (total internal reflection), strong multiple scattering across the fiber traps the beam in the transverse direction in the disordered fiber as the beam propagates in the longitudinal direction. An important difference between a conventional optical fiber and a disordered fiber of Ref. [20] is that the only bound modes in the conventional optical fiber are those confined to the core of the fiber; by contrast, transverse localization guides a beam launched at any point across the transverse profile of a disordered fiber. In an enclosed movie in Ref. [22], we showed that if the incoming beam of light is scanned across the input facet of the fiber, the outgoing beam follows the transverse position of the incoming beam and shifts its location. Here, we propose that this interesting property of the disordered fiber can be used in multiple-beam propagation for spatially multiplexed communication or imaging.

In order for the disordered fiber to be a viable medium for applications that benefit from spatial beam-multiplexing, we need to address two issues inherent in the design of these fibers. First, the localization mechanism is a statistical phenomenon based on multiple random scattering; therefore, the radius of the localized beam in the disordered optical fiber varies from position to position across the fiber. In the design presented in Ref. [20], this variation is approximately $15 \%$ of the average beam radius 
observed in the experiment. Fortunately, this variation can be reduced by increasing the index difference between the random sites of the disordered fiber or by operating at a shorter incident wavelength, as shown in Ref. [22]. The possibility of this reduction is rooted in the self-averaging behavior observed in this random process in the case of strong scattering $[20,38,39]$.

A disordered fiber with an index difference of 0.5 between the random sites (air voids in glass) was recently presented in Ref. [23]; however, the air void density was too low to reduce the variations in the beam radius. Ideally, the air void density must be near $50 \%$ [22] and further optimizations will be required in the future to improve the design.

Second, it is possible that the spatially multiplexed beams drift across the fiber when the fiber is subjected to substantial macro-bending. If the positions of the receivers at the output end of the fiber are initially spatially aligned with the multiple output beams of the ALOF, then the drift of the beams resulting from dynamic macrobending could result in misalignment and potential loss of the signal. The intention of this paper is to investigate the impact of macro-bending-induced drift in the center of the localized beams and show that it is possible to design Anderson localized optical fibers that can be used for practical beam-multiplexing applications. Our studies are mainly focused on the p-ALOF that was presented in Ref. [20], because it has the smallest localization radius among different samples that we have fabricated so far, and it allows us to compare our theoretical simulations with experimental observations. However, we numerically explore the beam walk-off effect in glass-air fibers as well, anticipating future interest in, and development of, these fibers [23]. 


\subsection{Multiple-beam propagation through a disordered}

\section{fiber}

In order to explore the propagation of multiple beams in a disordered fiber numerically, we use a finite difference beam propagation method (FD-BPM), as described in Ref. [22]. We choose the incident wavelength to be $405 \mathrm{~nm}$, which is also the wavelength of the laser used in our experiments. The refractive index distribution used for our numerical simulations is similar to that of the p-ALOF in Ref. [20], where $0.9 \mu \mathrm{m} \times 0.9 \mu \mathrm{m}$ sites are assigned refractive index values of $n_{1}=1.59$ or $n_{2}=1.49$ with equal probabilities. In order to observe a multiple-beam propagation effect in
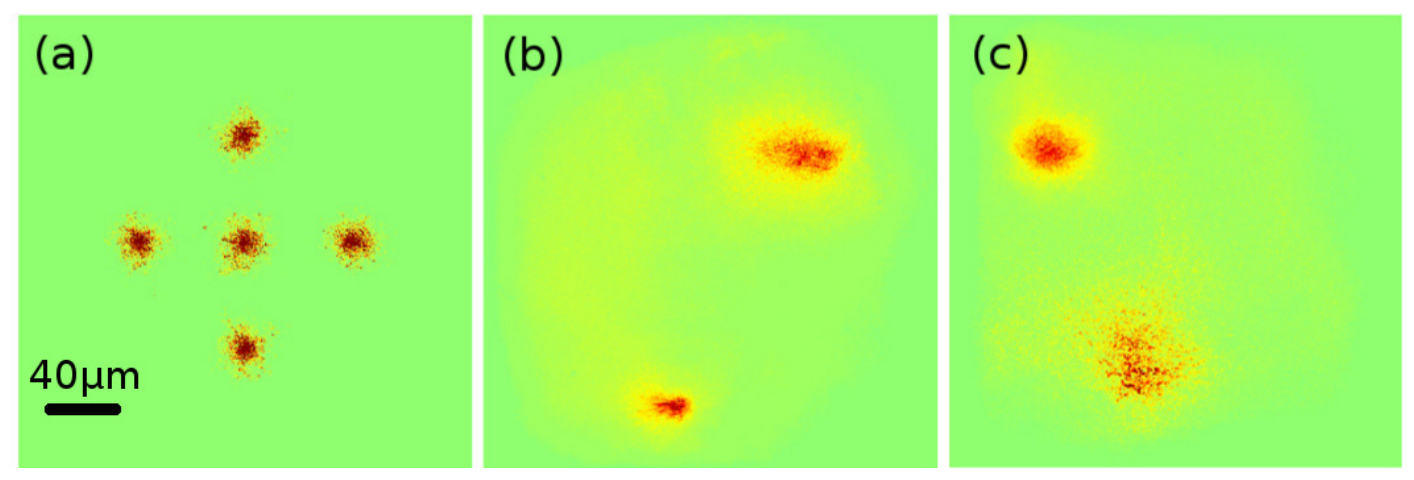

Figure 7.1: Multiple-beam propagation in a 5 cm-long p-ALOF (a) simulation for five beams; (b) experiment for two beams; and (c) experiment for two beams with different wavelengths. All beams are at $405 \mathrm{~nm}$ wavelength, except the bottom-middle beam in subfigure (c), which is at $633 \mathrm{~nm}$ wavelength.

a p-ALOF, we launch five incident beams, each with $2.4 \mu \mathrm{m}$ initial beam radius. In Fig. 7.1(a), we show the intensity profile after $5 \mathrm{~cm}$ of propagation along the fiber; the four exterior beams are launched at a distance of $70 \mu \mathrm{m}$ from the central beam. The output beams are observed to remain in the same spatial transverse position across the fiber as launched. In order to confirm our numerical observations, we carried out a similar experiment on a segment of p-ALOF, using the same procedure as in Ref. [20]. 
In Fig. 7.1(b), we show the output intensity from the p-ALOF, imaged on a CCD camera beam profiler using a 40x objective. The input double-beam is from two Thorlabs SMF630hp fibers, which are glued alongside each other after their jackets are stripped. We note that the cladding diameter of the SMF630hp fiber is $125 \mu \mathrm{m}$, and we estimate that the two cores were separated by about $190 \mu \mathrm{m}$, after the fibers were glued together.

Each fiber is illuminated separately using a $405 \mathrm{~nm}$ diode laser, and the double-fiber setup is butt-coupled to the p-ALOF sample that is $5 \mathrm{~cm}$ long. The measured output beam profile clearly illustrates that the two beams can be distinguished across the fiber in the output port.

In Fig. 7.1(c), we repeat the same experiment of Fig. 7.1(b), but replace one of the $405 \mathrm{~nm}$ diode laser sources with a He-Ne laser operating at $633 \mathrm{~nm}$ wavelength. In Ref. [22], we showed that the localized beam radius is larger for longer wavelengths. In Fig 7.1(c), the bottom-middle beam is at $633 \mathrm{~nm}$ wavelength and clearly has a larger localization radius than the top-left beam at $405 \mathrm{~nm}$ wavelength.

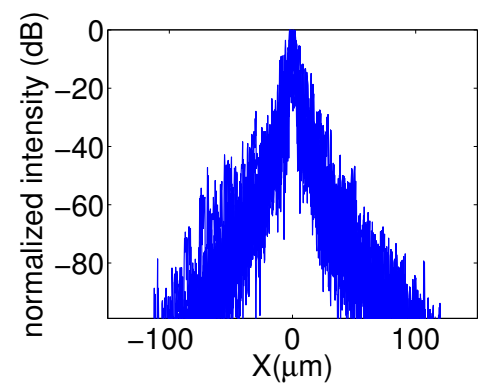

(a)

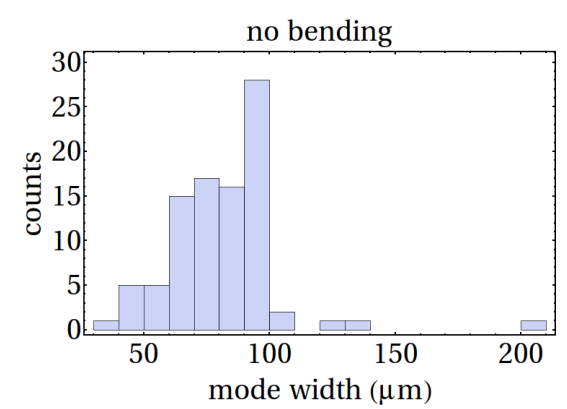

(b)

Figure 7.2: (a) The cross-section of the intensity profiles of the localized beam at $405 \mathrm{~nm}$ wavelength for 20 different realizations of the p-ALOF randomness are shown using numerical simulations, where the profiles are plotted on top of each other to capture the expected variations. (b) The experimental measurements of the beam width are shown in a histogram from 92 separate measurements. 
We note that the Anderson localization is a statistical phenomenon and the localization beam radius must be calculated by averaging over the elements of the "statistically" identical ensemble of random p-ALOFs. However, the large index difference between the random sites of the p-ALOF results in the "self-averaging" behavior (see, for example, Refs. $[38,39])$; therefore, similar levels of localization are observed for different randomly selected profiles. This self-averaging behavior is essential in ensuring that the $\mathrm{p}$-ALOF presented in here works as a true optical fiber (in the usual sense) and does not need to rely on statistical averaging of multiple samples to localize and guide the optical beam.

We note that despite the strong self-averaging behavior observed in p-ALOFs as also reported earlier in Refs. [20,22], some level of sample-to-sample variation remains and must be carefully studied, in order to ensure that our observations and conclusions hold well regardless of a specific random realization of the p-ALOF. In Fig. 7.2(a), we plot the intensity cross-section of the localized beam at $405 \mathrm{~nm}$ wavelength for 20 different realizations of the p-ALOF randomness using numerical simulations, where the localized beam intensity profiles are plotted on top of each other to help visualize the expected variations more clearly. We also note that the plot is presented in logarithmic scale to enhance the visual effect of the variation. The observations are in agreement with the previously reported results on the localization of the beam radius and the self-averaging behavior at the wavelength of $405 \mathrm{~nm}$ [22].

The numerical simulations in Fig. 7.2(a) can be compared with the experimental measurements presented in Fig. 7.2(b). In Fig. 7.2(b), we show a histogram of the experimental measurements of the beam width from 92 separate measurements. The data is collected by scanning the input beam from a piece of Thorlabs S405hp fiber over the tip of ten different p-ALOF samples and making nearly nine separate measurements for each fiber sample. We note that the general difference observed between simula- 
tion and experiment is consistent with imperfections in preparing the fiber samples (as explained below) and the noise in the CCD beam profiler at low intensities.

We note that in general, there are other parameters, besides the wavelength and the disorder strength, that can affect the variation of the localized beam radius across the fiber [22]. Other than the expected fluctuations due to the statistical nature of the localization, the quality of the fiber surface polishing and the local roughness can play an important role in sample-to-sample and region-to-region variations, especially in polymer-based fibers, where cleaving and polishing are more difficult.

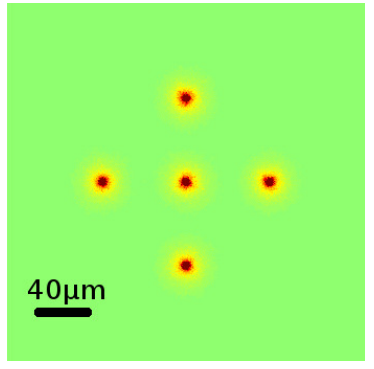

(a)

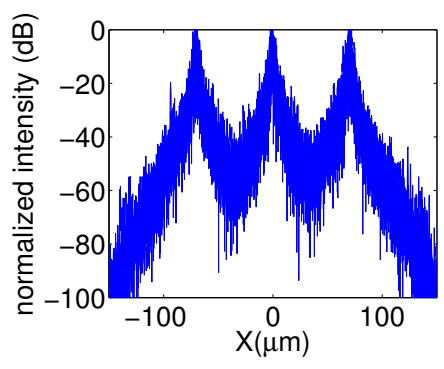

(b)

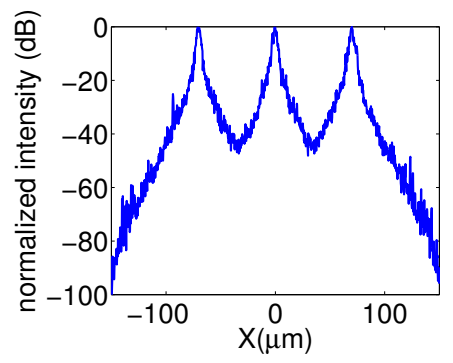

(c)

Figure 7.3: (a) Similar to Fig. 7.1(a), but the beam intensity is averaged over 20 different realizations of randomness. Substantial beam clean-up is observed compared with Fig. 7.1(a) due to the averaging. (b) Cross-section of the intensity profile where the results of 20 different realizations are plotted on top of each other to show the extent to which the beams overlap due to the statistical nature of the problem. (c) Same as (b) but the cross-sectional intensity is plotted for the average of the 20 different realizations. All figures are shown at $405 \mathrm{~nm}$ wavelength.

The five-beam intensity profile shown in Fig. 7.1(a) relates to a single simulation. Although the beams remain well-separated and localized due to the self-averaging behavior for different random realizations of the $\mathrm{p}-\mathrm{ALOF}$, we expect that the statistical averaging will reduce both the noise and the overlap between the beams. In Fig. 7.3(a), we show the five-beam intensity profile after averaging over 20 different simulations from a statistically identical ensemble of p-ALOFs; the averaged individual beams in Fig. 7.3(a) look considerably cleaner and more circularly symmetric compared with 
those from the single simulation of Fig. 7.1(a).

Although the individual beams in Figs. 7.1(a) and 7.3(a) look well-separated, this separation needs to be properly quantified, because making judgments solely based on the color scaling in such figures can sometimes be misleading. In order to verify the separation of the beams, we slice the beam intensity profile of Fig. 7.1(a) along the $\mathrm{x}$-axis at the center $(\mathrm{y}=0)$, and plot the cross section of the beam intensities in Fig. 7.3(b). In fact, in Fig. 7.3(b), we plot the results of 20 different random simulations on top of each other to show the extent of the possible variations due to randomness. Fig. 7.3(b) clearly shows that the exponentially decaying tails of the localized beams remain separated to better than $40 \mathrm{~dB}$ in intensity, which is beyond the dynamic range of the common CCD cameras. As we showed in Ref. [22], the localization radius of the beam in the $\mathrm{p}$-ALOF used in the present work is about $8 \mu m$ (based on numerical simulations) with the standard deviation of about $3 \mu m$ (at $405 \mathrm{~nm}$ wavelength); therefore, the beams are separated by 18 standard deviations, which is consistent with an intensity overlap of $40 \mathrm{~dB}$, considering the fluctuations around the average intensity.

In order to see the beam clean-up due to the averaging process, we take the 20 different random simulations of Fig. 7.3(b) and show their average in Fig. 7.3(c) (instead of plotting them on top of each other as we did in Fig. 7.3(b)). The averaged beam looks substantially cleaner with fewer fluctuations, as expected. We would like to emphasize that in real device realizations, one cannot likely rely on the ensemble averaging; the self-averaging must be strong enough to ensure that the localization and the beam separation can be observed in every element of the ensemble to the desired level. As shown here, statistical simulations are required to capture the degree of fluctuations in order to determine the minimum beam-to-beam separation given the device tolerance for the beam overlap. We note that the short wavelength of $405 \mathrm{~nm}$ used here helps in 
reducing the fluctuations due to strong self-averaging, as already discussed in Ref. [22].

\subsection{Impact of macro-bending on the drift of the cen- ter of localized beam}

As we discussed above, macro-bending can potentially result in a drift in the center of the localized beams in Anderson localized fibers. In order to investigate this drift, we use conformal mapping to model the bending of the optical fiber for our numerical simulations [64]. We assume that the fiber has a refractive profile of $n(x, y)$; when the fiber is bent, it can be conformally mapped to a straight fiber with a modified refractive index profile of $n^{\prime}(x, y)=n(x, y) \exp (-x / R)$. Here, it is assumed that the $\mathrm{p}$-ALOF is bent in the $\mathrm{x}$-transverse direction with a bend radius of $R$.

We note that according to Ref. [65], the elasto-optical coefficients should be included in the mapped refractive index profile for accurate modeling of the bend. In this work, we choose not to consider the elasto-optical effect; however, this choice will not impact our conclusions in this work.

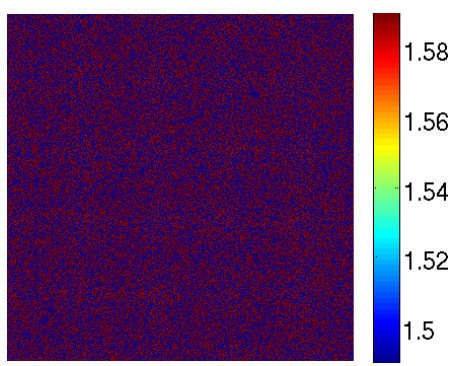

(a)

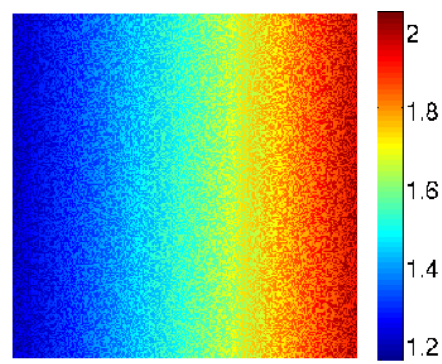

(b)

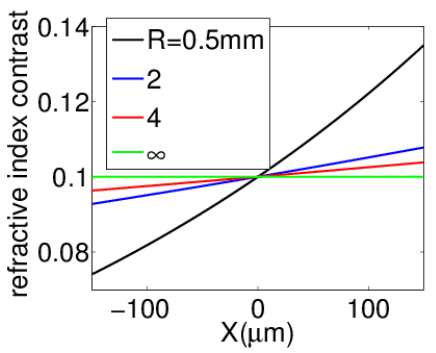

(c)

Figure 7.4: (a) Original index profile of the p-ALOF. (b) Conformally modified refractive index profile of a p-ALOF with bend radius of $0.5 \mathrm{~mm}$. (c) Effective refractive index difference between the low-index and high-index sites for different values of bend radius as a function of the location across the fiber profile. The fiber is assumed to be bent in the x-direction. The dimensions of subfigures (a) and (b) are $300 \mu m$ on each side. 
The original and modified refractive indices, $n(x, y)$ and $n^{\prime}(x, y)$ are compared in Figs. 7.4(a) and (b) for the bend radius of $R=0.5 \mathrm{~mm}$, for a sample p-ALOF. The side width of each square region shown in Fig. 7.4 is $d=300 \mu m$. Fig. 7.4(b) shows that the $n^{\prime}(x, y)$ varies considerably in the $\mathrm{x}$-direction due to the bending effect. While the refractive index structure is locally random, both the overall index and the local effective index differences between the random sites have a non-zero gradient due to the bending effect. Fig. 7.4(c) shows the effective refractive index difference between the low-index and high-index sites (after conformal mapping is included), for different values of bend radius, as a function of the location across the fiber profile.

We can now investigate whether the macro-bending can cause a drift in the location of the center of the localized beam. The center of the beam is defined in Ref. [66] and can shift if the bending effect is stronger than the localization effect. In Fig. 7.5(a), we show the "transverse" trajectory of the center of an optical beam across the fiber as the beam propagates along the p-ALOF for $5 \mathrm{~cm}$. For the numerical results shown in

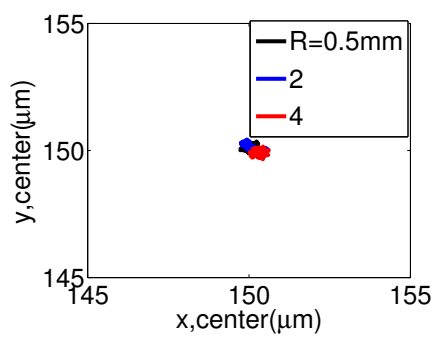

(a)

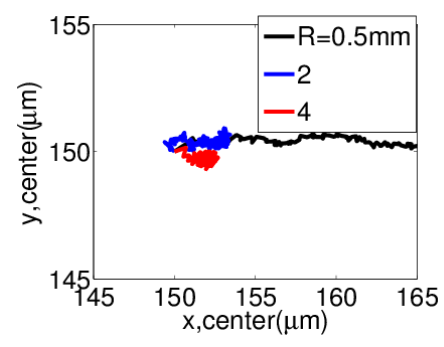

(b)

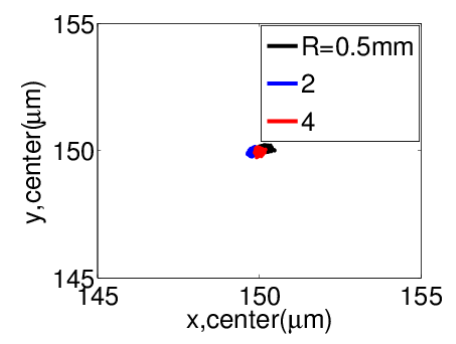

(c)

Figure 7.5: Trajectory of the beam center across the fiber as the beam propagates along a $5 \mathrm{~cm}$ segment for different bend radii in a) polymer fiber at $\lambda=405 \mathrm{~nm}$, b) polymer fiber at $\lambda=633 \mathrm{~nm}, \mathrm{c})$ glass fiber at $\lambda=633 \mathrm{~nm}$.

Fig. 7.5(a), the wavelength is $405 \mathrm{~nm}$ and the bend radius is $R=0.5 \mathrm{~mm}, R=2 \mathrm{~mm}$, and $R=4 \mathrm{~mm}$. The small bend-radius of $R=0.5 \mathrm{~mm}$ is a worst-possible scenario for a reasonable practical application. For all values of the bend radius, no serious walkoff is observed even after $5 \mathrm{~cm}$ of propagation and Anderson localization is observed 
to dominate over macro-bending.

In Fig. 7.5(b), we carry out a similar numerical experiment, but at a longer wavelength, $633 \mathrm{~nm}$. As discussed in Ref. [22], transverse Anderson localization is weaker for longer wavelengths. Therefore, in Fig. 7.5(b) we observe that macro-bending dominates the localization effect, more so for $R=0.5 \mathrm{~mm}$ (black line) than $R=2 \mathrm{~mm}$ (blue line) and $R=4 \mathrm{~mm}$ (red line). For the same wavelength, $633 \mathrm{~nm}$, we expect to see that the transverse Anderson localization will dominate the effects of macro-bending, if the index contrast is raised, as explained in Ref. [22]. In Fig. 7.5(c), we carry out a numerical experiment similar to that of Fig. 7.5(b), yet with a larger index difference between the random sites $\left(n_{1}=1.5, n_{2}=1.0\right)$, and observe no serious walk-off in the trajectory of the beam center over $5 \mathrm{~cm}$ of propagation, regardless of the bend radius. We note that when there is substantial walk-off, the beam does not preserve its shape and develops a considerable ellipticity in its profile in the direction of the bend [53].

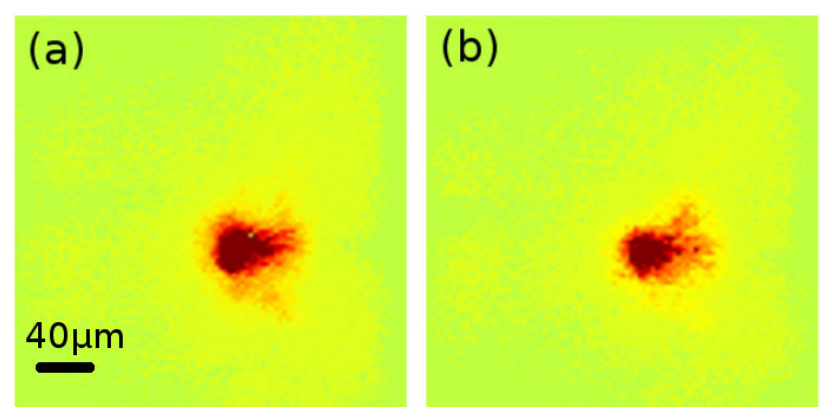

Figure 7.6: Experimental measurement of the intensity of the propagated light in a fiber with (a) no bend, (b) bend radius of $1 \mathrm{~mm}$. The wavelength is $405 \mathrm{~nm}$ and the fiber sample is $15 \mathrm{~cm}$ long. No shift is observed, which is also consistent with the simulations in Fig. 7.5(a). We have intentionally saturated the CCD camera slightly to illustrate the location of the beams with respect to the boundary of the fiber for easier comparison.

In order to verify our numerical calculations for the drift of the beam center, we carry out an experiment on a sample p-ALOF, where the results are shown in Figs. 7.6(a) and (b). In Fig. 7.6(a), we measure the output beam profile of the propagated Anderson localized beam at $405 \mathrm{~nm}$ wavelength in a $15 \mathrm{~cm}$-long $\mathrm{p}$-ALOF. We then bend the 
fiber with a bend radius of approximately $1 \mathrm{~mm}$ and measure the beam profile again, as shown in Fig. 7.6(b). The bend is applied to a $10 \mathrm{~cm}$ section of the $15 \mathrm{~cm}-\mathrm{long}$ p-ALOF, resulting in 16 turns; the remaining $5 \mathrm{~cm}$ was left to hold the fiber in the set-up. We observe no noticeable beam walk-off effect, which is also consistent with our numerical simulations in Fig. 7.5(a).

We note that the numerical results presented in Figs. 7.5(a), 7.5(b), 7.5(c) and the experimental results in Fig. 7.6 are each for a single realization of the randomness in the fiber without any averaging. In order to confirm that the localization in the bent p-ALOF at $405 \mathrm{~nm}$ wavelength survives multiple realizations of the random profile, we plot in Fig. 7.7 a histogram of the experimental measurements of the beam width from 72 separate measurements in bent fibers with the bend radius of approximately $1 \mathrm{~mm}$. We remind that the variations are due to both the statistical variations of the localization phenomenon as well as the imperfections in preparing the fiber samples (such as variations in polishing).

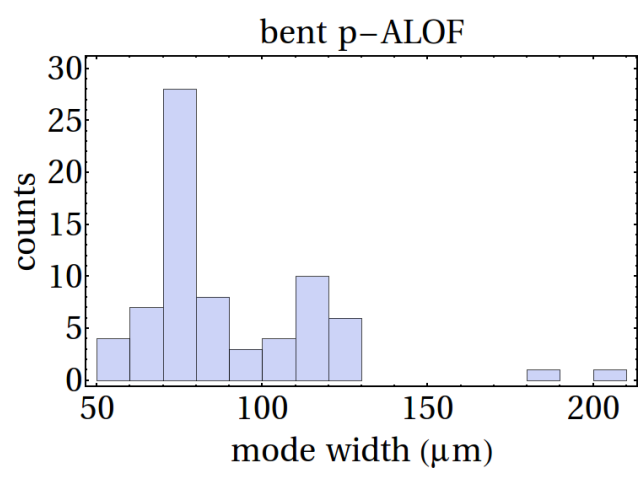

Figure 7.7: Histogram of the experimental measurements of the beam width from 72 separate measurements in bent fibers with the bend radius of approximately $1 \mathrm{~mm}$. The localization behavior holds for the majority of the 72 random realizations explored in this figure.

We showed in Fig. 7.4(c) that the effective index difference between random sites varies across the bent fiber. Therefore, we expect the localization effect to be stronger in the region with a higher index difference, i.e., the inside of the bend. In order to observe 
this effect numerically, we launch three separate beams in a $5 \mathrm{~cm}$ fiber bent with a bend radius of $0.5 \mathrm{~mm}$, where one beam is close to the inside of the bend (Fig. 7.8(a)), another is launched at the center of the fiber (Fig. 7.8(b)), and the other is close to the outside of the bend (Fig. 7.8(c)). The output profiles clearly show that the output beam in Fig. 7.8(c) has the smallest localization radius due to a larger effective index difference between the random sites of the p-ALOF. In Fig. 7.8(d), we compare the cross section of the averaged intensity for 20 realizations of randomness, where the red profile is related to the less-localized beam near the inside of the bend and the blue profile is related to the more-localized beam near the outside of the bend. We note that in the numerical experiments quoted above, the distance of each beam is $70 \mu \mathrm{m}$ from the center of the fiber, directly across the bending coordinate (which is the $\mathrm{x}$-axis). We note that the relative beam intensity is plotted down to $-100 \mathrm{~dB}$ to clearly show the difference in the localization effect. However, in practice, it may be difficult to experimentally observe this difference between the localization radii, especially if the difference is smaller than or comparable to the variations inherent in the statistical nature of Anderson localization. This is obviously the case for p-ALOF. Moreover, $0.5 \mathrm{~mm}$ is smaller than any practically interesting value of the bend radius.

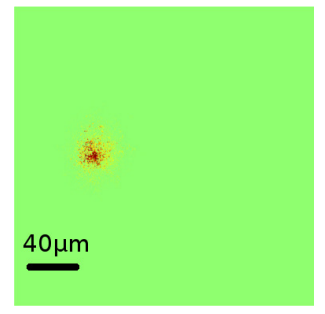

(a)

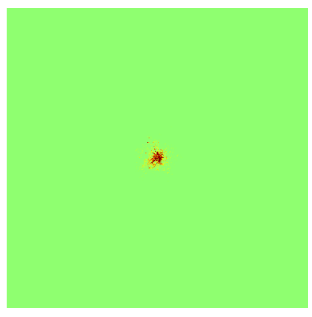

(b)

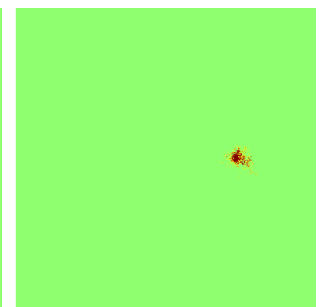

(c)

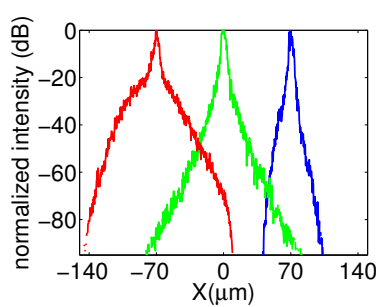

(d)

Figure 7.8: Beam intensity of the propagated light after $5 \mathrm{~cm}$ of propagation in a bent pALOF with $R=0.5 \mathrm{~mm}$, when the light is launched closer to the (a) inside of the bend, (b) center of the fiber, and (c) outside of the bend. (d) Cross section of the beam intensity averaged over 20 samples for the beam in subfigure (a) in red versus the beam in subfigure (b) in green color versus the beam in subfigure (c) in blue color. 


\subsection{Conclusion}

We have shown, both numerically and experimentally, that a p-ALOF can effectively work as a multicore optical fiber. We have also shown that not only can we still observe transverse Anderson localization in a bent disordered fiber, but when the refractive index difference between the random sites is sufficiently large compared with the change in the effective index produced by the bent fiber across the beam, there is also no substantial shift in the center of the beam for reasonable values of the bend radius.

Although averaging over different realizations of the random p-ALOF results in a higher quality of localization, one cannot rely on such ensemble averaging in practical device applications; rather, the self-averaging must be strong enough to ensure that the localization and the beam separation can be observed in every element of the ensemble to the desired level. In the p-ALOFs studied here, the beam overlap was shown to be suppressed to better than $40 \mathrm{~dB}$ for the beam-to-beam separation of $70 \mu m$ using numerical simulations. In the measured samples, the tails of the beams usually extended farther than predicted by theory, most likely because of imperfections in preparing the fiber samples. We also show experimentally that the beam profiles remain well-localized at $405 \mathrm{~nm}$ wavelength even when the fibers are bent with a bend radius of around $1 \mathrm{~mm}$. Future efforts are focusing on glass ALOFs with the site-tosite refractive index difference of 0.5 that can result in robust localization of multiple beams at wavelengths longer than $405 \mathrm{~nm}$. 


\section{Chapter 8}

\section{Image transport through the}

\section{disordered polymer optical fiber}

\section{mediated by transverse Anderson}

\section{localization}

\subsection{Introduction}

Anderson localization is the absence of diffusive wave propagation in certain disordered media [1-3,67]. Transverse Anderson localization was first introduced by Abdullaev, et al., [14] and De Raedt, et al., [13]. In the treatment suggested by De Raedt, et al., an optical wave system is studied in which the refractive index profile is random in the transverse plane and is invariant in the longitudinal direction. An optical beam launched in the longitudinal direction can become localized in the transverse plane due to the strong random scattering from the transverse random index fluctuations 
and can propagate in the longitudinal direction with a finite beam radius, similar to a conventional optical fiber. The radius of the localized beam depends on the extent of the fluctuations in the value of the refractive index, the characteristic length over which the fluctuations occur, as well as the wavelength of the light [20, 22, 24].

Transverse Anderson localization was first observed experimentally in 2007 [15]. That experiment, carried out in a photo-refractive crystal, utilized refractive index variations of the order of $10^{-4}$. Such small variations in the refractive index of random sites result in a very large mean value and standard deviation of the localized beam radius [22]. For device applications that benefit from the waveguiding properties of transverse Anderson localization, the mean localized beam radius should be comparable with or smaller than that of conventional optical fibers and large sample-to-sample variations in the beam radius are not acceptable. Therefore, it is necessary to increase the refractive index difference of the random sites.

We recently demonstrated transverse Anderson localization of light in a polymer random optical fiber medium with refractive index fluctuations of order 0.1 [20]. The polymer Anderson localized fiber (p-ALOF) allowed for the simultaneous propagation of multiple beams in a single strand of disordered optical fiber [24] with potential applications in beam-multiplexed optical communications and optical imaging.

In this work, we present what is, to the best of our knowledge, the first demonstration of optical image transport using transverse Anderson localization of light, specifically in a disordered optical fiber. The possibility of using disordered optical fiber for some form of image transport was expected, given the earlier demonstration of spatial beam multiplexing in p-ALOF [24]. The novelty of the presented work is in demonstrating that the image transport quality can be of a comparable or higher quality than the commercially available multicore imaging optical fibers. It is remarkable that the high 
quality image transport is achieved because of, not in spite of, the high level of disorder and randomness in the imaging system.

Multicore optical fibers have been used extensively in high resolution optical imaging [68]; however, the transmitted images are inherently pixelated due to the discrete nature of the light-guiding array of cores, and the inter-core coupling can reduce the image contrast and result in blurring $[69,70]$. Certain structural nonuniformities such as variations in the size of the cores were shown by Ref. [71] to improve the image transport quality. A weakly disordered fiber array was also studied in Ref. [72] and was shown to induce diffusive spreading or localization at a few sites across the fiber. High numerical aperture guiding cores were also suggested by Ref. [70] to reduce coreto-core coupling and blurring in imaging applications.

A highly disordered optical fiber with large refractive index fluctuations can transport high quality images, as it provides a high degree of structural nonuniformity as well as a sufficiently large local numerical aperture. More rigorously, the image transport quality is due to the transverse Anderson localization phenomenon that creates localized transport channels with finite radii (localized optical modes) through the disordered imaging waveguide $[17,35]$. A higher amount of disorder and a larger level of fluctuation in the refractive index provides stronger beam localization, hence improving the image resolution. It is also responsible for the reduction in the value of the standard deviation in the localized beam radius as a consequence of the selfaveraging behavior $[20,38,39]$, ensuring uniform image transport quality across the fiber facet. The coherent transverse coupling and blurring is considerably reduced, because the transverse disorder results in strong spatial incoherence across the beam, akin to using incoherent light to eliminate speckles in an imaging system. Therefore, even a laser can be readily used for illumination in this image fiber to obtain a higher signal-to-noise ratio, without worrying about its undesirably high spatial coherence. 
The fabrication of the p-ALOF used in these imaging experiments is described in detail in Refs. [19,20]; briefly, it is composed of 40,000 strands of poly methyl methacrylate (PMMA) and 40,000 strands of poly styrene (PS) drawn to a square profile with a side width of $250 \mu \mathrm{m}$ and site sizes of about $0.9 \mu \mathrm{m}$. A magnified scanning electron microscope (SEM) image of a portion of the tip of the p-ALOF is shown in Fig. 8.1(a) where the PMMA sites (refractive index of 1.49) are darker compared with the lighter PS sites (refractive index of 1.59). Also shown in Fig. 8.1(b), for comparison, is an SEM image of a portion of the tip of a glass disordered fiber earlier reported in Ref. [23], where the darker sites are the air voids. In the following, we demonstrate highquality optical image transport through the p-ALOF related to Fig. 8.1(a) mediated by transverse Anderson localization of light. We also argue that a higher disorder density is required for quality image transport through the glass disordered fiber of Fig. 8.1(b) and a higher air void fill-fraction of nearly $50 \%$ should result in a very high-quality image fiber.
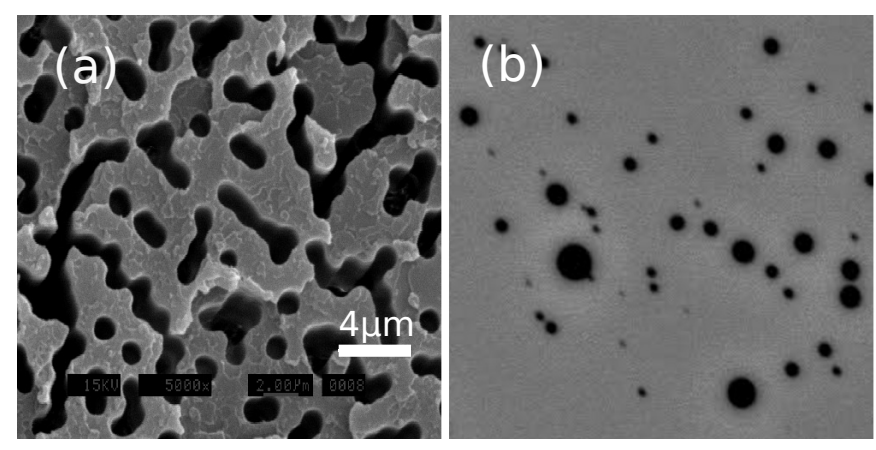

Figure 8.1: Magnified SEM image of a portion of the tip of the (a) p-ALOF and (b) glass disordered fiber. For p-ALOF in (a), the PMMA (PS) sites are darker (lighter) in color. For the glass disordered fiber, the darker sites are the air voids. The $4 \mu \mathrm{m}$ scale-bar applies to both images. 


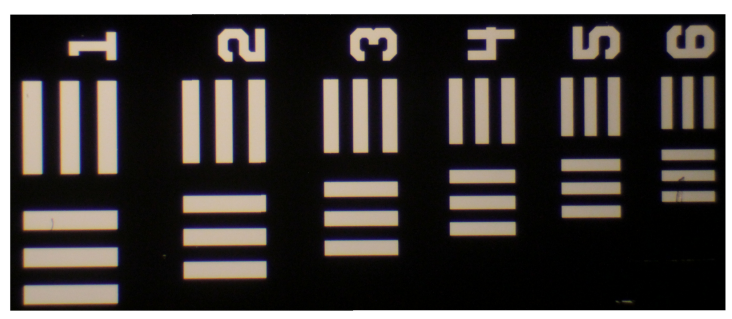

Figure 8.2: Elements of a group on 1951 U.S. Air Force test target (1951-AFTT).

\subsection{Image transport in the disordered fiber}

In order to investigate the image transport capability of the p-ALOF, optical images of the "numbers" from group 3 and group 4 on an 1951 U.S. Air Force resolution test chart (1951-AFTT), Fig. 8.2, are launched using a $405 \mathrm{~nm}$ laser diode into the p-AOLF.

For imaging, we chose fiber samples of nearly $5 \mathrm{~cm}$ long and imaged a section of 1951 U.S. Air Force resolution test target (R1DS1N from Thorlabs) by directly buttcoupling the test target to the polished input end of the p-ALOF. The test target was directly illuminated by a light source. The near-field output was imaged using 40x and 60x objectives onto a lensed CCD camera. Different numbers in each group are the same size.

The transported images for the groups 3 and 4 are shown in Fig. 8.3 and Fig. 8.4, respectively, after $5 \mathrm{~cm}$ of propagation in the p-ALOF. Theoretically, the minimum resolution of the images is determined by the width of the point spread function of the p-ALOF imaging setup, which is comparable with the localization length. The transverse localization length (localized beam radius) of the p-ALOF at $405 \mathrm{~nm}$ wavelength was calculated to be smaller than $10 \mu \mathrm{m}$ [22]. In practice, the imaging resolution in p-ALOF is limited by the quality of the cleave and polishing of the p-ALOF surface. The fiber surface quality both at the input and output is partially responsible for the 
distortions observed in the transported images in Figs. 8.3 and 8.4.

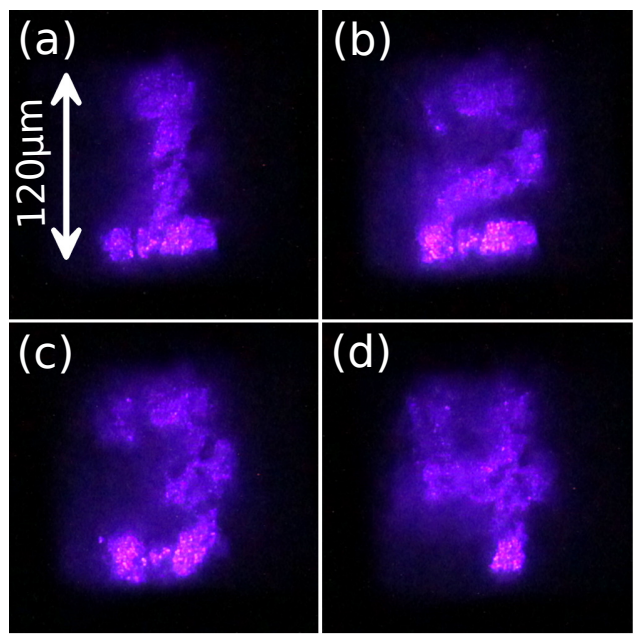

Figure 8.3: Transported images of different numbers through $5 \mathrm{~cm}$ of p-ALOF, (a)-(d), related to the group 3 on the 1951-AFTT (experimental measurements).

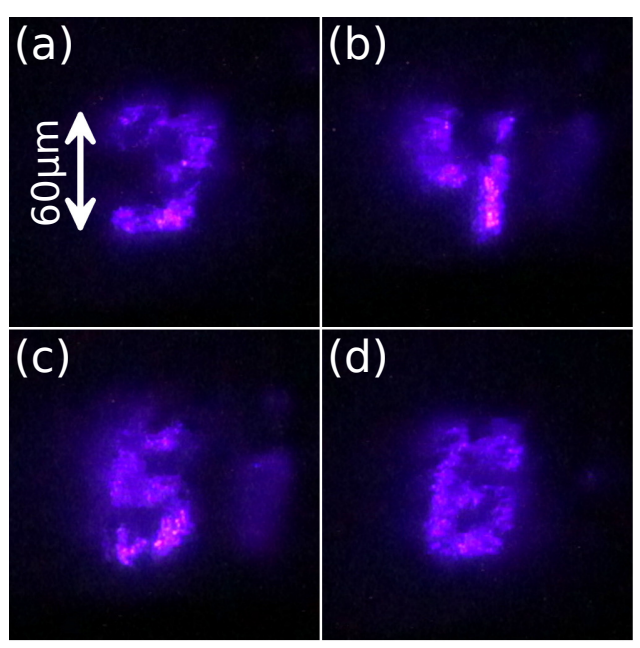

Figure 8.4: Transported images of the numbers through $5 \mathrm{~cm}$ of p-ALOF, (a)-(d), related to the group 4 on the 1951-AFTT (experimental measurements).

\subsection{Image quality assessment metrics}

The most legitimate approach for determining the quality of an image that is viewed by a human eye is the subjective evaluation. However, subjective evaluation can be 
time consuming, expensive, and impractical. Objective image quality assessment is used to closely approximate the subjective perception. Objective measures such as the mean squared error (MSE) and the peak signal to noise ratio (PSNR) [73,74] are developed for automatic evaluation of perceived images.

MSE is the most widely used and simplest signal quality/fidelity measure [75]. It is computed by averaging the squared error between the pixel intensities of distorted and reference images

$$
M S E=\frac{1}{M \times N} \sum_{i=1}^{N} \sum_{j=1}^{M}\left(X_{i, j}-Y_{i, j}\right)^{2},
$$

where $M$ and $N$ are the horizontal and vertical pixel counts of the two images, $X$ and $Y$, that are being compared. In the field of image processing, MSE is usually mapped to a peak signal to noise ratio (PSNR) that takes into account the dynamic range $(L)$ of pixel intensities

$$
P S N R=10 \log _{10} \frac{L^{2}}{M S E}
$$

Despite the simplicity and clear physical meaning of MSE/PSNR, their quality assessment are not a good representation of the visually perceived quality and may result in large errors in quality assessment [73].

A highly effective method in the assessment of image quality is the structural similarity index (SSIM) that is developed based on the assumption that human visual system is greatly adapted for extracting structural information [73]. In SSIM, the quality of the transported images are compared with the distortion free initial images that are launched into the fibers, by correlating the local pixel intensity patterns in different regions of the two images.

SSIM is used to compare the local image patches $x$ and $y$ from the same locations of 
the two images [75]. A simplified form of SSIM index is calculated using

$$
\operatorname{SSIM}(x, y)=\frac{\left(2 \mu_{x} \mu_{y}+C_{1}\right)\left(2 \sigma_{x y}+C_{2}\right)}{\left(\mu_{x}^{2}+\mu_{y}^{2}+C_{1}\right)\left(\sigma_{x}^{2}+\sigma_{y}^{2}+C_{2}\right)}
$$

where $\mu_{x}$ and $\mu_{y}$ are local sample means of $x$ and $y, \sigma_{x}$ and $\sigma_{y}$ are local sample standard deviation of $x$ and $y$, and $\sigma_{x y}$ is the cross correlation of samples $x$ and $y$ after subtracting their mean. $C_{1}$ and $C_{2}$ are positive numbers to stabilize the SSIM in cases of near zero mean, standard deviation or cross correlation. $C_{1}$ and $C_{2}$ are defined as $\left(K_{1} L\right)^{2}$ and $\left(K_{2} L\right)^{2}$ where $L$ is the dynamic range of the pixel intensities and $K_{1} \ll 1$, $K_{2} \ll 1$.

In order to compare the quality of the transported images in the disordered optical fibers with the periodic imaging fibers, the quality of the transported images in both fibers are determined by mean structural similarity index (MSSIM). The MSSIM index is calculated by averaging the SSIM index

$$
M S S I M=\frac{1}{N} \sum_{j=1}^{N} \operatorname{SSIM}\left(x_{j}, y_{j}\right)
$$

where $N$ is the number of local patches of the image and $x_{j}$ and $y_{j}$ are the image contents at the $j$ th patch.

\subsection{Comparison with commercial multicore image fibers}

The imaging performance of the p-ALOF compares with some of the best commercially available multicore imaging optical fibers, as shown in Fig. 8.5. The transported images over $5 \mathrm{~cm}$ of the number "6" from group 5 of the 1951-AFTT test chart are compared between p-ALOF in Fig. 8.5(a), Fujikura FIGH-10-350S in Fig. 8.5(b), and 
Fujikura FIGH-10-500N in Fig. 8.5(c). The visual image quality of the transported image through the p-ALOF is clearly better than FIGH-10-350S and is comparable with FIGH-10-500N. We would like to emphasize that the feature sizes in Fig. 8.5 are of the order of 10-20 $\mu \mathrm{m}$. The Rayleigh range for this level of resolution and the $405 \mathrm{~nm}$ laser wavelength is approximately $0.8-3 \mathrm{~mm}$, which is substantially shorter than the $5 \mathrm{~cm}$ propagation length in these image fibers. Therefore, the imaging results are non-trivial and cannot be obtained using bulk propagation or conventional multimode optical fibers.

Mean structural similarity index (MSSIM) is used here as a quantitative measure to closely approximate the perceived image quality by human eye [73, 75]. The MSSIM values are 0.5877 for Fig. 8.5(a), 0.5501 for Fig. 8.5(b), and 0.5591 for Fig. 8.5(c).

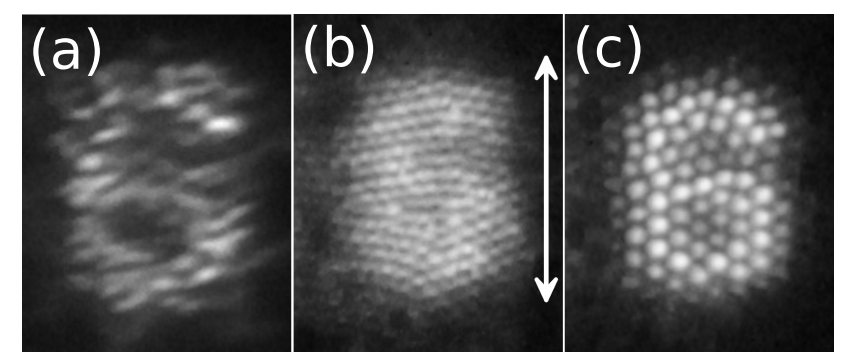

Figure 8.5: Transported images related to group 5 of the 1951-AFTT test chart in (a) p-ALOF, (b) FIGH-10-350S image fiber and (c) FIGH-10-500N image fiber (experimental measurements). The scale bar in (b) is $30 \mu \mathrm{m}$-long and the same scale bar can be used for (a) and (c). Each fiber is approximately $5 \mathrm{~cm}$ long. The MSSIM image quality values for the images are: (a) 0.5877, (b) 0.5501, and (c) 0.5591.

Using the optical and physical parameters of the p-ALOF, Fujikura FIGH-10-350S, and Fujikura FIGH-10-500N, we repeat the experiment of Fig. 8.5 using the numerical simulation and show the results in Fig. 8.6(a), Fig. 8.6(b), and Fig. 8.6(c).

The images are propagated along the fiber by numerically solving the wave propagation equation described in Ref. [22]. The illuminated image "UWM" and number " 6 " are created using the GIMP image editor and used as the input. The disordered refractive 
index profile is created by randomly assigning the refractive indices of $n_{1}$ and $n_{2}$ to the different sites. The sites are square with the side width of $0.9 \mu \mathrm{m}$.

Similar to Fig. 8.5, the transported images are over $5 \mathrm{~cm}$ of the number "6" created using the GIMP image editor and nearly the same size as the group 5 of the 1951-AFTT test chart and are compared between p-ALOF in Fig. 8.6(a), Fujikura FIGH-10-350S in Fig. 8.6(b), and Fujikura FIGH-10-500N in Fig. 8.6(c).

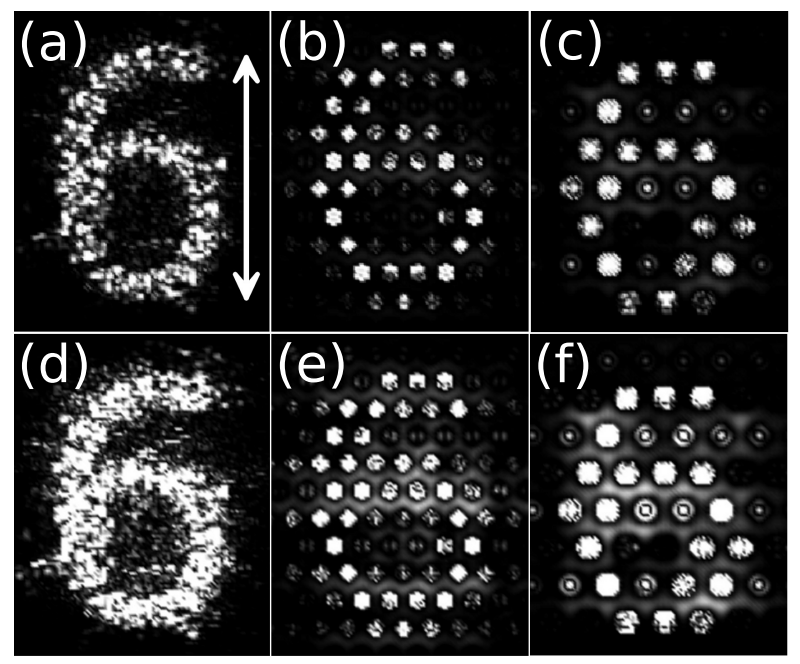

Figure 8.6: Transported images related to group 5 of the 1951-AFTT test chart in (a) p-ALOF, (b) FIGH-10-350S image fiber and (c) FIGH-10-500N image fiber (numerical simulations). The scale bar in (a) is $30 \mu \mathrm{m}$-long and the same scale bar can be used for other subfigures. Each fiber is approximately $5 \mathrm{~cm}$ long. The top row (subfigures (a), (b), and (c)) can be compared with the experimental results shown in Fig. 8.5. Subfigures (d), (e), and (f) in the bottom row are the same as Subfigures (a), (b), and (c) in the top row, except the images are saturated by changing the color axis in gray-scale colormap in Matlab. The color axis of $[0,1]$ in subfigures (a), (b), and (c) is changed to color axis of [0,0.3] in (d), (e), and (f). The MSSIM image quality values for the images are: (a) 0.637 , (b) 0.615 , and (c) 0.6257 . The MSSIM values for (d), (e), and (f) are the same as (a), (b), and (c), respectively.

Similar to the experimental results of Fig. 8.5, the numerical simulation shown in Fig. 8.6 indicates that the visual image quality of the transported image through the p-ALOF is better or comparable with the Fujikura image fibers. The experiment and numerics are in reasonable agreement, but there are differences, as well. Possible sources of difference can be traced back to uncertainties in relating the experiment 
to numerics. The MSSIM values are 0.637 for Fig. 8.6(a), 0.615 for Fig. 8.6(b), and 0.6257 for Fig. 8.6(c).

\subsection{Sources of systematic image degradation}

In the experiment, a good precision in the butt-coupling of the test target to the input fiber is needed to obtain high-quality output images. An important source of uncertainty is the surface quality of the fibers, determining the precision in coupling the input profile from the test target and coupling the output to the CCD camera. The Fujikura image fibers are cleaved and polished using commercial-grade equipment, while the p-ALOF is hand-cleaved and polished using the lapping paper from Thorlabs. These variations and uncertainties cannot be easily accounted for in the numerical simulation without extensive surface quality characterizations of p-ALOF and Fujikura image fibers and are likely not very illuminating, considering that the p-ALOF images can be improved if specialized equipment for cleaving and polishing polymer fibers are used to improve its surface quality. Another possible source of uncertainty is the degree of spatial coherence of the laser used to illuminate the test target. The spectral bandwidth of the laser also contributes the fuzziness of the experiment, while the single-frequency numerical simulation looks more pixelated.

We note that the scalar wave equation has been benchmarked extensively with the full vectorial beam propagation method and the results have shown to be in excellent agreement for the numerical apertures relevant in these simulations [22].

The quality of the imaging setup and the saturation of the CCD camera versus the saturation of the images generated using numerics can be another source of perceived difference between the experiment and numerics. The images of Fig. 8.6(a), Fig. 8.6(b), 
and Fig. 8.6(c) are plotted using the color axis of [0,1] in gray-scale colormap in Matlab. In Fig. 8.6(d), Fig. 8.6(e), and Fig. 8.6(f), the color axis is changed to [0,0.3] for the same simulation, resulting in a more fuzzy image. It should also be noted that the numerical simulations are for images at the tip of the fiber, while those from the experiment are after passing through the near-field imaging setup.

The simulated images of multicore image fibers in Figs. 8.6 look quite different from the experiment in Fig. 8.5, even when one takes into account the uncertainties mentioned above. This issue was pointed out in Ref. [71], where it was suggested that the discrepancy between theory and experiment is because the image fibers are not composed of identical cores. The published core size specification is likely the average value with potentially a large standard deviation. While it is possible to build a concrete model to investigate this issue in detail, it is beyond the scope and interests of this work.

\subsection{Disorder-induced localization is responsible for enhanced image transport}

The discussion so far has mainly focused on the perceived quality of the images in Fig. 8.5 and Fig. 8.6. Because the intention of this work is to argue that transverse Anderson localization induced by the disorder results in a higher quality image transport when compared with multicore image fibers, it is important to ensure that similar specifications are used for the disordered and multicore image fibers to ensure that the comparison is conducted fairly. The core and cladding refractive indices of the Fujikura image fibers are 1.5 and 1.446, respectively, for which the index difference of 0.054 is lower than that of $\mathrm{p}-\mathrm{ALOF}(0.1)$. In order to isolate the effect of disorder and 
Anderson localization on the image transport quality and eliminate the differences due to the numerical apertures, an image fiber with the same numerical aperture as p-ALOF and the structural parameters of FIGH-10-500N is modeled using the numerical simulation. The input image is the word "UWM," where each letter is $44 \mu \mathrm{m}$ high, the lines in the letters are $6 \mu \mathrm{m}$ thick, and the letters are separated by $26 \mu \mathrm{m}$. The intensity profiles of the transported images at the wavelength of $405 \mathrm{~nm}$ after $5 \mathrm{~cm}$ of propagation in $\mathrm{p}-\mathrm{ALOF}$ and the image fiber with a raised numerical aperture are compared in Fig. 8.7(a) and 8.7(b). The quality of the transported image in Fig. 8.7(a) is higher than that of the modified image fiber in Fig. 8.7(b). There is considerable pixelation in Fig. 8.7(b) and a low intensity halo of illuminated cores fills the area in between the main lines of the transported letters. It appears that even with the same level of index difference, p-ALOF provides a comparable or better quality image transport compared with the multicore image fiber. It should be noted

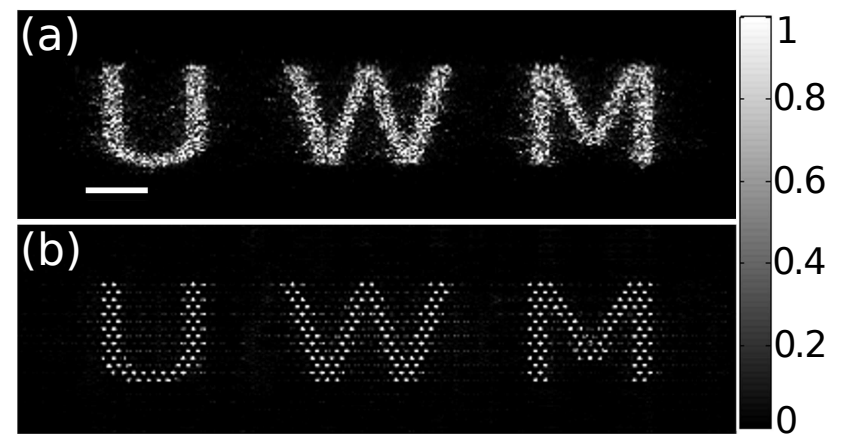

Figure 8.7: The intensity profile of the "UWM" image after $5 \mathrm{~cm}$ of propagation and at the wavelength of $405 \mathrm{~nm}$ in a (a) p-ALOF, (b) modified image fiber with the refractive index difference of 0.1 between the cores and the clad, using numerical simulations. The scale bar in (a) is $20 \mu \mathrm{m}$ long and the same scale bar can be used for (b). The MSSIM image quality value for the images are: (a) 0.8923 and (b) 0.6263 .

that the images in Fig. 8.7 appear to be of higher quality compared with Figs. 8.5 and 8.6, simply because they are of different sizes and resolutions. The MSSIM values are 0.8923 for Fig. 8.7(a) and 0.6263 for Fig. 8.7(b).

As further concrete evidence that the disorder is responsible for the higher image trans- 
port quality, the quantitative MSSIM metric for image quality assessment is compared in Fig. 8.8 for a collection of multicore image fibers with and without disorder. The blue squares in Fig. 8.8 represent the value of MSSIM for image transport through $5 \mathrm{~cm}$ of disorder-free $(\Delta=0)$ periodic image fiber as a function of the periodicity $\Lambda$. The radius of each individual core is $1.45 \mu \mathrm{m}$, which is the same as the mean radius of the cores in the FIGH-10-500N imaging fiber. The refractive index difference between the cores and common cladding is 0.1 . The image that is transported through the image fiber is the number "6" created using the GIMP image editor and is $65 \mu \mathrm{m}$ high. In the case of disorder-free periodic image fiber, MSSIM is low for small $\Lambda$ as expected because of the enhanced core-to-core coupling. It is also low for large $\Lambda$ due to the pixelation effect. In general, the optimum $\Lambda$ for the highest value of MSSIM depends on the numerical aperture and the radius of the individual core.

The red circles and cyan diamonds in Fig. 8.8 represent the value of MSSIM for image transport through disordered $(\Delta \neq 0)$ image fibers. The radius of each individual core is chosen from a uniform distribution in the interval $[1.45 \mu m-\Delta, 1.45 \mu m+\Delta]$. Therefore, the mean radius is the same as that of the periodic case. $\Delta=0.3 \mu \mathrm{m}$ for red circles and $\Delta=0.9 \mu m$ for cyan diamonds. For each image fiber, the value of MSSIM is calculated using the transported image. The numerical experiment is repeated 30 times for each representation of the disorder (at each $\Lambda$ and $\Delta$ ), in order to obtain sufficient statistics, where the error-bars in MSSIM signify one standard deviation around the mean MSSIM.

It is observed that at each value of $\Lambda$, the presence of the disorder improves the quality of the transported image. A detailed analysis shows that the maximum value of MSSIM is obtained for $\Lambda=3.9 \mu \mathrm{m}$ and $\Delta=0.3 \mu \mathrm{m}$. Although the disordered multicore image fiber explored in here differs from the structure of the p-ALOF, this numerical experiment provides further evidence in support of the claim that the pres- 
ence of disorder can enhance the quality of the image transport.

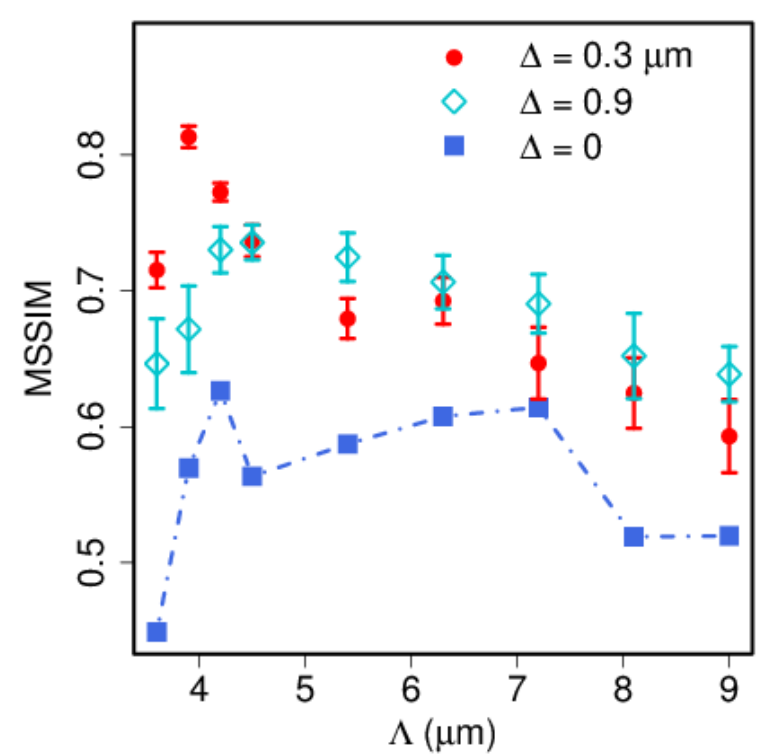

Figure 8.8: The MSSIM metric for image quality assessment is compared for periodic image fibers as a function of the core periodicity $\Lambda$. The blue squares represent disorder-free $(\Delta=0)$ periodic cores of radius $1.45 \mu \mathrm{m}$. The red circles and cyan diamonds represent disordered $(\Delta \neq 0)$ periodic cores of mean radius $1.45 \mu \mathrm{m}$ with uniform random radius variations in the interval $[-0.3 \mu \mathrm{m}, 0.3 \mu \mathrm{m}]$ and $[-0.9 \mu \mathrm{m}, 0.9 \mu \mathrm{m}]$, respectively.

\subsection{Disorder improves image transport quality}

In Fig. 8.8, the MSSIM metric for image quality assessment is compared for periodic image fibers as a function of the core periodicity $\Lambda$. A sample refractive index profile of a disorder-free $(\Delta=0)$ periodic structure with the core-to-core distance of $\Lambda=3.9 \mu \mathrm{m}$ is shown in Fig. 8.9(a). The refractive index profile of a randomized structure with $\Delta=0.3 \mu \mathrm{m}$ is also shown in Fig. 8.9(b). To randomize the periodic structure, the core radii are randomly chosen from a uniform distribution in the range of $[r-\Delta, r+\Delta]$, where $r=1.45 \mu \mathrm{m}$ is the mean core radius, $\Delta=0.3$ represents the variation in the radius. Samples of the transported images in the disorder-free periodic fiber and the 


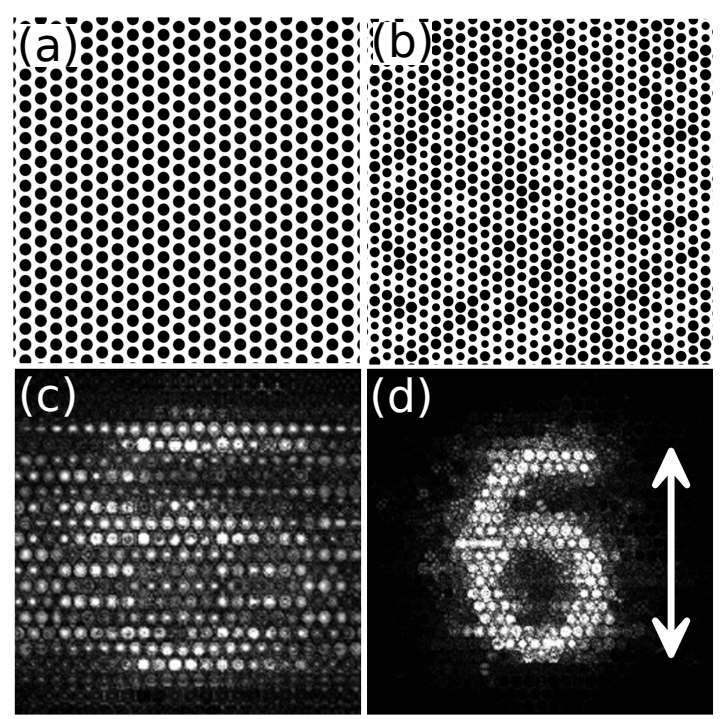

Figure 8.9: (a) The refractive index profile of a periodic structure with the cores radii of $1.45 \mu \mathrm{m}$ and the core to core distances of $3.9 \mu \mathrm{m}$. (b) The disordered structure with the variation of $\Delta=0.3 \mu \mathrm{m}$ in its cores radii. (c) The transported image after $5 \mathrm{~cm}$ of propagation in a periodic fiber with the refractive index profile in (a). (d) The transported image after $5 \mathrm{~cm}$ of propagation in a periodic fiber with the refractive index profile in (b). The scale bar in (d) is $65 \mu \mathrm{m}$ and the same scale bar can be used for (a)-(c).

disordered structure with $\Delta=0.3 \mu \mathrm{m}$ are shown in the Figs. $8.9(\mathrm{c})$ and $8.9(\mathrm{~d})$, respectively.

\subsection{Image quality versus propagation distance}

In Fig. 8.10, we plot the image quality assessment metric MSSIM as a function of propagation distance for the same input image and fiber parameters as of the fibers reported in Fig. 8.9, as well as the disordered polymer transverse Anderson localized fiber (p-ALOF). The blue squares represent the disorder-free $(\Delta=0)$ periodic multicore image fiber with the core-to-core distance of $\Lambda=3.9 \mu \mathrm{m}$, and the red circles represent the disordered case of $\Delta=0.3$. In both cases, the image quality drops in the first few millimeters of propagation and then saturates near the final value. The 


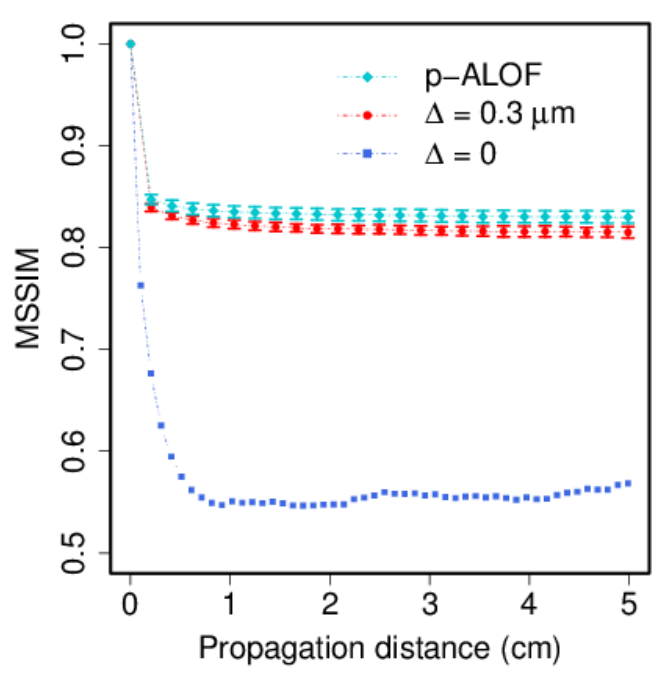

Figure 8.10: MSSIM is plotted as a function of propagation distance for the disorder-free $(\Delta=0)$ periodic multicore image fiber in blue squares, the disordered case of $\Delta=0.3$ in red circles, and the disordered polymer Anderson localized fiber p-ALOF in cyan diamonds.

saturated MSSIM is considerably larger for the disordered fiber. The error-bars indicate one standard deviation around the mean MSSIM for the disordered fiber for an ensemble of 30 simulations.

The cyan diamonds represent the value of MSSIM for image propagation through the p-ALOF. The MSSIM values are higher than the best disordered multicore image fiber with $\Delta=0.3$. This presents another concrete evidence that the $\mathrm{p}$-ALOF is comparable or better than conventional multicore image fibers. We emphasize that the value of MSSIM depends on the size of the transported image and the MSSIM numbers reported in Fig. 8.10 should only compared with that of images with the same size. 


\subsection{Impact of the wavelength on image quality}

For image transport through multicore imaging optical fibers, a longer optical wavelength increases the inter-core coupling strength and therefore lowers the quality of image transport. A similar effect can be observed in Anderson localized optical fibers as the mean localized beam radius has been shown to be larger for a longer wavelength [22].

In Fig. 8.11, a longer wavelength of $633 \mathrm{~nm}$ is used for the numerical simulation of the image transport. The results must be compared with Fig. 8.6. Fig. 8.11 shows the numerical simulation of transported images related to group 5 of the 1951-AFTT test chart in (a) FIGH-10-350S image fiber, (b) FIGH-10-350S image fiber, and (c) pALOF, using the $633 \mathrm{~nm}$ wavelength. The quality of the transported image at $633 \mathrm{~nm}$ is substantially lower than at $405 \mathrm{~nm}$ wavelength for all cases. For p-ALOF, the localization radius was calculated to be smaller than $10 \mu \mathrm{m}$ at $405 \mathrm{~nm}$ wavelength and nearly $30 \mu m$ at $633 \mathrm{~nm}$ wavelength [22]. Because the localization radius determines the image transport resolution in a disordered optical fiber, the resolution is nearly three times lower at $633 \mathrm{~nm}$ than at $405 \mathrm{~nm}$ wavelength. For image fibers, longer wavelength results in a larger modal overlap between the cores, hence increasing coreto-core coupling and blurring of the image. It must be noted that the quality for imaging through the p-AOLF at the wavelength of $633 \mathrm{~nm}$ is still higher than that of the multicore image fibers at the same wavelength. The MSSIM values are 0.3385 for Fig. 8.11(a), 0.5396 for Fig. 8.11(b), 0.6119 for Fig. 8.11(c), and 0.6509 for Fig. 8.11(d). 


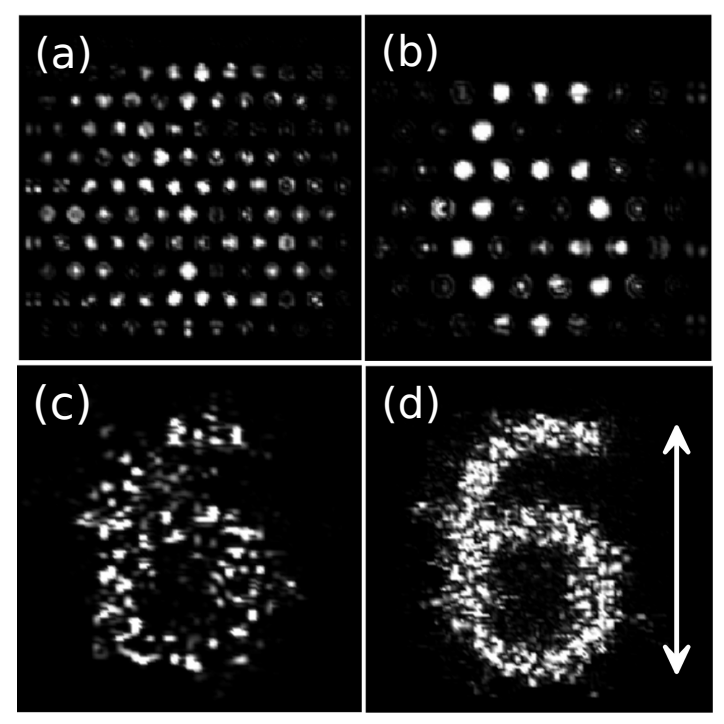

Figure 8.11: Numerical simulation of transported images related to group 5 of the 1951AFTT test chart in (a) FIGH-10-350S image fiber, (b) FIGH-10-500N image fiber, and (c) p-ALOF. Each fiber is approximately $5 \mathrm{~cm}$ long. The simulation wavelength is $633 \mathrm{~nm}$ and lower quality imaging is obtained, as expected, when compared with Fig. 8.6 at $405 \mathrm{~nm}$ wavelength. (d) is similar to (c), except an air-glass material with the index difference of 0.5 is assumed instead of 0.1 related to the polymer p-ALOF of (c). The scale bar in (d) is $20 \mu \mathrm{m}$ long and the same scale bar can be used for (a), (b), and (c). The MSSIM image quality value for the images are: (a) 0.3385, (b) 0.5396, (c) 0.6119, and (d) 0.6509 .

\subsection{Impact of the disorder refractive index differ- ence on image quality}

A higher value of the refractive index difference between the random sites in a disordered fiber such as p-ALOF is expected to improve the image transport quality by decreasing the localized beam radius [22]. An ideal disordered image fiber is made from a glass matrix with wavelength-size randomly distributed air voids to provide a refractive index contrast of 0.5 between high- and low-index sites. The ideal air void fill-fraction is at $50 \%$ for maximum transverse scattering and minimum localized beam radius [22]. In Fig. 8.11(d), we show the image transport through a disordered fiber with the same geometrical parameter of $\mathrm{p}$-ALOF, but the refractive index values of 
the high- and low-index sites are set at 1.5 and 1.0, respectively, for the wavelength of the $633 \mathrm{~nm}$. The quality of image transport in Fig. 8.11(d) is substantially better than Fig. 8.11(c).

We note that not only is the average localized beam radius lower in a properly designed glass-air disordered optical fiber compared with a p-ALOF, but there also are fewer sample-to-sample variations in the localized beam radius. Therefore, a higher image transport quality is expected with a more uniform performance across the fiber.

\subsection{Signal attenuation along the fiber}

Much lower intrinsic attenuation is expected in silica-based fibers compared with polymer fibers for longer image transport. In fact, $16 \mathrm{~cm}$ has been the longest $\mathrm{p}$-ALOF sample that has been successfully used for imaging; transported images of the numbers "1" and "6" from group 3 of the 1951-AFTT test chart are shown in Fig. 8.12. The maximum length for image transport in the p-ALOF is both a result of optical attenuation and the variations in the side width of the optical fiber in the draw process. White light has been used for the image transport in Fig. 8.12, and the output images appear red because the attenuation of the polymer materials used in p-ALOF is minimum for the red color in the visible spectrum. The large variations in the side width of the optical fiber in the draw process and the accumulated dust and humidity during the week-long stacking of the fibers due to the considerable static electrical charges that build up on the polymer fibers result in a loss of around $0.5 \mathrm{~dB} / \mathrm{cm}$ at $633 \mathrm{~nm}$. However, it is expected that by assembling the fibers in a clean-room environment and using a more stable draw process, the length of p-ALOF image fibers can be extended to at least several meters. 
A glass-air disordered optical fiber would be an ideal solution to the attenuation problem. Moreover, it is possible to obtain high quality fiber-end surfaces by using standard cleaving and polishing equipment in the laboratory. For the glass-air disordered fibers reported in Ref. [23] and shown in Fig. 8.1(b), transverse Anderson localization can be observed only near the boundary, because their air fill-fraction is $2-5 \%$ in the central regions, which is far below the ideal 50\%; even near the boundary where transverse Anderson localization is observed, the localized beam radius is too large to be suitable for image transport.

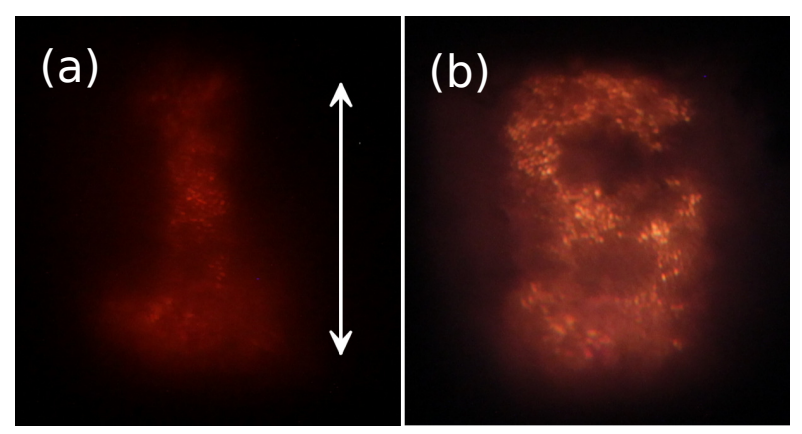

Figure 8.12: Transported images of the numbers " 1 " and "6" from group 3 of the 1951AFTT test chart through a $16 \mathrm{~cm}$-long p-ALOF sample are shown using a white light source (experimental measurements). The scale bar in (a) is $120 \mu \mathrm{m}$ long and the same scale bar can be used for (b).

\subsection{Comparison with other advanced fiber-based imag- ing methods}

Finally, it would be important to compare the advantages and disadvantages of imaging using the disordered optical fibers with other available fiber-based techniques. For example, Choi, et al., [76], recently developed a highly interesting technique to use a single-core multimode optical fiber for endoscopic imaging. They relied on preand post-processing techniques to evaluate the transmission matrix of a multimode 
optical fiber to address the modal dispersion. Also, speckle imaging and turbid lens imaging methods are used to eliminate distortions. The post-processing limits the image acquisition time to one frame per second. In comparison, the disordered fiber provides a one-to-one map between the object and image plane and no additional optics or pre- and post-processing is required to obtain the image. Therefore, image acquisition time is negligible.

Another advantage of the disordered fiber-imaging over the technique presented by Choi, et al., [76], is that their method does not support a fully flexible endoscopic operation due to the variation of the transmission matrix induced by the bending and twisting of the fiber. The beam localization in disordered fiber is very robust, even in very tight bends as shown in Ref. [24], so it can easily operate in a fully flexible endoscopic system.

The main advantage of the method of Ref. [76] over the disordered fiber-imaging is the imaging resolution. They report 12300 effective image pixels over the $200 \mu \mathrm{m}$ diameter core of the fiber, hence a pixel size of $6.4 \lambda^{2}$. Based on our simulations, even the best glass-air disordered fibers can only achieve pixel resolution of larger than $\sim 30 \lambda^{2}$, which is lower than that reported by Choi, et al. On the other hand, the disordered fiber is easily scalable, so it is conceivable the fabricate disordered fibers with millimeter-size cross sections for ultra-wide-area and nearly instantaneous imaging for streaming videos, while the post-processing would make other techniques prohibitively slow. It must also be noted that the disordered fiber is inherently a multimode fiber and the technique employed by Choi, et al., can be applied to the disordered fiber as well. In that case, the localized nature of the beam transport and also the direct knowledge of transported images, albeit at a slightly lower resolution, can potentially reduce the post-processing time in their technique. 


\subsection{Conclusion}

In conclusion, we present what is, to the best of our knowledge, the first demonstration of optical image transport using transverse Anderson localization of light in a polymer disordered optical fiber (p-ALOF first reported in Ref. [20]). The image transport quality is comparable with or better than some of the best commercially available multicore image fibers with less pixelation and higher contrast. In practice, the imaging resolution in $\mathrm{p}$-ALOF is limited by the quality of the cleave and polishing of the p-ALOF surface, and the maximum transport distance is limited by the optical attenuation as well as the variations in the side width of p-ALOF in the draw process. The ultimate disordered image fiber will be made from a glass matrix with wavelength-size randomly distributed air voids with an air void fill-fraction of $50 \%$. The low optical attenuation in glass-air material is essential for transporting images over longer distances than reported here. The large index difference between the glass matrix and the air voids and 50\% air void fill-fraction provide maximum scattering in the transverse plane to reduce the localization beam radius and to minimize the width of the imaging point spread function. The large transverse scattering is also responsible for reducing the beam-to-beam variation of the localization radius. A small value of the standard deviation is essential for device applications, as one expects to observe a nearly uniform width of the point spread function across the tip of the image fiber as well as among fiber samples. The reduction in the value of the standard deviation of the localized beam radius is due to the self-averaging behavior observed in the presence of strong scattering $[20,38,39]$. In general, Anderson localization is a statistical problem and the localization happens only strictly when averaged over many elements of a statistically identical ensemble. However, the large transverse scattering results in a strong self-averaging behavior where the localized beam radius of each element 
of the ensemble is nearly equal to the average localized beam radius; therefore, the standard deviation is small. Further details on the self-averaging property of highly scattering disordered fibers can be found in Ref. [22].

Future efforts are focused on designing an air-glass disordered fiber with $50 \%$ air void fill-fraction, where the average size of the individual air voids will be using numerical simulations optimized to obtain the minimum beam localization radius. We note that image transport has been investigated previously in random phase-separated glasses $[77,78]$. Unlike the p-ALOF, their reported structure lacks longitudinal invariance. Fabrication of an ideal air-glass disordered fiber is very challenging using the stack-and-draw techniques, considering that 100,000 or more elements must be used and the air voids must remain open after the draw. It may be possible to achieve this using a lower number of sites and by restacking/redrawing to obtain the required sub-micron resolution of the disorder. It may also be possible to use porous glass such as that reported in Ref. [23], albeit with a higher air void fill-fraction of nearly $50 \%$. Future efforts will also include revisiting image transport in random phase-separated glasses and its relationship to transverse Anderson localization. 


\section{Bibliography}

[1] P. W. Anderson, "Absence of diffusion in certain random lattices" Phys. Rev. 109, 1492-1505 (1958).

[2] P. Sheng, Introduction to Wave Scattering, Localization, and Mesoscopic Phenomena, (Academic Press, 1995).

[3] S. John, "Electromagnetic absorption in a disordered medium near a photon mobility edge," Phys. Rev. Lett. 53, 2169-2172 (1984).

[4] P. W. Anderson, "The question of classical localization: a theory of white paint?," Phil. Mag. B. 52, 505-509 (1985).

[5] S. John, "Strong localization of photons in certain disordered dielectric superlattices," Phys. Rev. Lett. 58, 2486-2489 (1987).

[6] A. D. Lagendijk, B. van Tiggelen, and D. S. Wiersma, "Fifty years of Anderson localization," Phys. Today 62, 24-29 (2009).

[7] A. F. Ioffe and A. R. Regel, "Non-crystalline, amorphous and liquid electronic semiconductors," Prog. Semicond. 4, 237-291 (1960).

[8] A. A. Chabanov, M. Stoytchev, and A. Z. Genack, "Statistical signatures of photon localization," Nature 404, 850-853 (2000). 
[9] R. Dalichaouch, J. P. Armstrong, S. Schulz, P. M. Platzman, and S. L. McCal, "Microwave localization by two-dimensional random scattering," Nature 354, 53$55(1991)$.

[10] M. Störzer, P. Gross, C. M. Aegerter, and G. Maret, "Observation of the critical regime near Anderson localization of light," Phys. Rev. Lett. 96, 063904 (2006).

[11] D. S. Wiersma, P. Bartolini, A. Lagendijk and R. Righini, "Localization of light in a disordered medium," Nature 390, 671-673 (1997).

[12] H. Hu, A. Strybulevych, J.H. Page, S.E. Skipetrov and B.A. van Tiggelen, "Localization of ultrasound in a three-dimensional elastic network," Nature Physics 4, 945-948 (2008).

[13] H. De Raedt, A. D. Lagendijk, and P. de Vries, "Transverse localization of light," Phys. Rev. Lett. 62, 47-50 (1989).

[14] S. S. Abdullaev and F. Kh. Abdullaev, "On propagation of light in fiber bundles with random parameters," Radiofizika 23, 766-767 (1980).

[15] T. Schwartz, G. Bartal, S. Fishman, and M. Segev, "Transport and Anderson localization in disordered two-dimensional photonic lattices," Nature 446, 52-55 (2007).

[16] M. Segev, Y. Silberberg, and D. N. Christodoulides, "Anderson localization of light ," Nat. Photonics 7, 197-204 (2013).

[17] Y. Lahini, A. Avidan, F. Pozzi, M. Sorel, R. Morandotti, D. N. Christodoulides, and Y. Silberberg, "Anderson localization and nonlinearity in one-dimensional disordered photonic lattices," Phys. Rev. Lett. 100, 013906 (2008). 
[18] J. C. Butcher, Numerical methods for ordinary differential equations, (Wiely, second edition, 2008).

[19] S. Karbasi, R. J. Frazier, K. W. Koch and A. Mafi, "Fabrication and characterization of two-dimensional Anderson localized polymer optical fibers," J. Vis. Exp. 77, e50679 (2013).

[20] S. Karbasi, C. R. Mirr, P. G. Yarandi, R. J. Frazier, K. W. Koch, and A. Mafi, "Observation of transverse Anderson localization in an optical fiber," Opt. Lett. 37, 2304-2306 (2012).

[21] S. Johnson and J. Joannopoulos, "Block-iterative frequency-domain methods for Maxwell's equations in a planewave basis," Opt. Express 8, 173-190 (2001).

[22] S. Karbasi, C. R. Mirr, Ry. J. Fraizer, P. G. Yarandi, K. W. Koch, and A. Mafi, "Detailed investigation of the impact of the fiber design parameters on the transverse Anderson localization of light in disordered optical fibers," Opt. Express 20, 18692-18706 (2012).

[23] S. Karbasi, T. Hawkins, J. Ballato, K. W. Koch, and A. Mafi, "Transverse Anderson localization in a disordered glass optical fiber," Opt. Mater. Express 2, 1496-1503 (2012).

[24] S. Karbasi, K. W. Koch, and A. Mafi, "Multiple-beam propagation in an Anderson localized optical fiber," Opt. Express 21, 305-313 (2013).

[25] S. Karbasi, R. J. Frazier, K. W. Koch, T. Hawkins, J. Ballato, and A. Mafi, "Image transport through a disordered optical fiber mediated by transverse Anderson localization," arXiv:1307.4160, accepted in Nature Communications (2014). 
[26] W. P. Huang and C. L. Xu, "Simulation of three-dimensional optical waveguides by full-vector beam propagation method," J. Lightwave Technol. 29, 2639-2649 (1993).

[27] H. B. Keller, Analysis of Numerical Methods, (Courier Dover Publications, 1994).

[28] D. Markiewicz, "Survey on Symplectic Integrators," Preprint Univ. California at Berkeley, (1999).

[29] G. R. Hadley, "Transparent boundary condition for the beam propagation method," IEEE J. Quantum Electron. 28, 363-370 (1992).

[30] B. E. A. Saleh, M. C. Teich, Fundamentals of Photonics, (Wiley-Interscience, 2 edition. March 9, 2007).

[31] www.comsol.com

[32] L. Martin, G. Di Giuseppe, A. Perez-Leija, R. Keil, F. Dreisow, M. Heinrich, S. Nolte, A. Szameit, A. F. Abouraddy, D. N. Christodoulides, and B. E. A. Saleh, "Anderson localization in optical waveguide arrays with off-diagonal coupling disorder," Opt. Express 19, 13636-13646 (2011).

[33] A. Szameit, Y. V. Kartashov, P. Zeil, F. Dreisow, M. Heinrich, R. Keil, S. Nolte, A. Tünnermann, V.A. Vysloukh, and L. Torner, "Wave localization at the boundary of disordered photonic lattices," Opt. Lett. 35, 1172-1174 (2010).

[34] O. Abdi, K. C. Wong, T. Hassan, K. J. Peters, and M. J. Kowalsky, "Cleaving of solid single mode polymer optical fiber for strain sensor applications," Opt. Commun. 282, 856-861 (2009). 
[35] S. Karbasi, K. W. Koch, and A. Mafi, "Modal perspective on the transverse Anderson localization of light in disordered optical lattices," J. Opt. Soc. Am. B 30, 1452-1461 (2013).

[36] Paradigm Optics, Incorporated, Vancouver, WA 98682 USA.

[37] M. Leonetti and C. López, "Measurement of transport mean-free path of light in thin systems," Opt. Lett. 36, 2824-2826 (2011).

[38] J. M. Ziman, Models of Disorder, (Cambridge University Press, 1979).

[39] M. V. Berry, and S. Klein, "Transparent mirrors: rays, waves and localization," Eur. J. Phys. 18, 222-228 (1997).

[40] T. Philbin, C. Kuklewicz, S. Robertson, S. Hill, F. Konig, and U. Leonhardt, "Fiber-optical analog of the event horizon," Science 319, 1367-1370 (2008).

[41] J. C. Knight, T. A. Birks, P. S. J. Russell, and D. M. Atkin, "All-silica single-mode optical fiber with photonic crystal cladding," Opt. Lett. 21, 1547-1549 (1996).

[42] T. A. Birks, J. C. Knight, and P. S. J. Russell, "Endlessly single-mode photonic crystal fiber," Opt. Lett. 22, 961-963 (1997).

[43] J. C. Knight, J. Arriaga, T. A. Birks, A. Ortigosa-Blanch, W. J. Wadsworth, and P. St. J. Rus- sell, "Anomalous dispersion in photonic crystal fiber," IEEE Photon. Tech. Lett. 12, 807- 809 (2000).

[44] J. K. Ranka, R. S. Windeler, and A. J. Stentz, "Visible continuum generation in air-silica microstructure optical fibers with anomalous dispersion at $800 \mathrm{~nm}, "$ Opt. Lett. 25, 25-27 (2000).

[45] J. C. Knight, J. Broeng, T. A. Birks, and P. St. J. Russell, "Photonic band gap guidance in optical fibers," Science 20, 1476-1478 (1998). 
[46] S. Coleman, Aspects of symmetry: selected Erice lectures, (Cambridge University Press, 1985).

[47] F. Bloch, "Uber die quantenmechanik der elektronen in kristallgittern," Z. Physik 52, 555-600 (1928).

[48] P. A. Lee and T. V. Ramakrishnan, "Disordered electronic systems," Rev. Mod. Phys. 57, 287-337 (1985).

[49] S. Ghosh, B. P. Pal, R. K. Varshney, and G. P. Agrawal, "Transverse localization of light and its dependence on the phase front curvature of the input beam in a disordered optical waveguide lattice," J. Opt. 14, 075701-075705 (2012).

[50] S. Ghosh, N. D. Psaila, R. R. Thomson, B. P. Pal, R. K. Varshney, and A. K. Kar, "Ultrafast laser inscribed waveguide lattice in glass for direct observation of transverse localization of light," Appl. Phys. Lett. 100, 101102-101105 (2012).

[51] D. M. Jović, Y. S. Kivshar, C. Denz, and M. R. Belic, "Anderson localization of light near boundaries of disordered photonic lattices," Phys. Rev. A 83, 033813033817 (2011).

[52] M. Rechtsman, A. Szameit, F. Dreisow, M. Heinrich, R. Keil, S. Nolte, and M. Segev, "Amorphous photonic lattices: band gaps, effective mass, and suppressed transport," Phys. Rev. Lett. 106, 193904 (2011).

[53] Y. V. Kartashov, V. V. Konotop, V. A. Vysloukh, and L. Torner, "Light localization in nonuniformly randomized lattices," Opt. Lett. 37, 286-288 (2012).

[54] N. Otsu, "A threshold selection method from gray-level histograms," IEEE Trans. Syst. Man. and Cybern. SMC-9, 62-66 (1979). 
[55] S. Iano, T. Sato, S. Sentsui, T. Kuroha, and Y. Nishimura, "Multicore optical fiber," in Optical Fiber Communication, 1979 OSA Technical Digest Series (Optical Society of America, 1979), paper WB1.

[56] B. Rosinski, J. W. D. Chi, P. Grosso, and J. Le Bihan, "Multichannel transmission of a multicore fiber coupled with vertical-cavity surface-emitting lasers," J. Lightwave Technol. 17, 807-810 (1999).

[57] J. M. Fini, B. Zhu, T. F. Taunay, and M. F. Yan, "Statistics of crosstalk in bent multicore fibers," Opt. Express 18, 15122-15129 (2010).

[58] B. Zhu, T. F. Taunay, M. F. Yan, J. M. Fini, M. Fishteyn, E. M. Monberg, and F. V. Dimarcello, "Seven-core multicore fiber transmissions for passive optical network," Opt. Express 18, 11117-11122 (2010).

[59] T. Xie, D. Mukai, S. Guo, M. Brenner, and Z. Chen, "Fiber-optic-bundle-based optical coherence tomography," Opt. Lett. 30, 1803-1805 (2005).

[60] R. K. Kostuk and J. Carriere, "Interconnect characteristics of fiber image guides," Appl. Opt. 40, 2428-2434 (2001).

[61] C. Amatore, A. Chovin, P. Garrigue, L. Servant, N. Sojic, S. Szunerits, and L. Thouin, "Remote fluorescence imaging of dynamic concentration profiles with micrometer resolution using a coherent optical fiber bundle," Anal. Chem. 76, $7202-7210(2004)$

[62] A. F. Gmitro and D. Aziz, "Confocal microscopy through a fiber-optic imaging bundle," Opt. Lett. 18, 565-567 (1993).

[63] K. L. Reichenbach and C. Xu, "Numerical analysis of light propagation in image fibers or coherent fiber bundles," Opt. Express 15, 2151-2165 (2007). 
[64] M. Heiblum and J. H. Harris, "Analysis of curved optical waveguides by conformal transformation," IEEE J. Quantum Electron. 11, 75-83, (1975).

[65] R. T. Schermer and J. H. Cole, "Improved bend loss formula verified for optical fiber by simulation and experiment," IEEE J. Quantum Electron. 43, 899-909, (2009).

[66] A. Mafi and J. V. Moloney, "Beam quality of photonic crystal fibers," J. Lightwave Technol. 23, 2267-2270 (2005).

[67] J. Billy, V. Josse, Z. Zuo, A. Bernard, B. Hambrecht, P. Lugan, D. Clément, L. Sanchez-Palencia, P. Bouyer, and A. Aspect, "Direct observation of Anderson localization of matter waves in a controlled disorder," Nature 453, 891-894 (2008).

[68] H. H. Hopkins and N. S. Kapany, "A flexible fiberscope, using static scanning," Nature 173, 39-41 (1954).

[69] J.-H. Han, J. Lee, and J. U. Kang, "Pixelation effect removal from fiber bundle probe based optical coherence tomography imaging," Opt. Express 18, 7427-7439 (2010).

[70] X. Chen, K. L. Reichenbach, and C. Xu, "Experimental and theoretical analysis of core-to-core coupling on fiber bundle imaging," Opt. Express 16, 21598-21607 (2008).

[71] K. L. Reichenbach and C. Xu, "Numerical analysis of light propagation in image fibers or coherent fiber bundles," Opt. Express 15, 2151-2165 (2007).

[72] T. Pertsch, U. Peschel, J. Kobelke, K. Schuster, H. Bartelt, S. Nolte, A. Tünnermann, and F. Lederer, "Nonlinearity and disorder in fiber arrays," Phys. Rev. Lett. 93, 053901 (2004). 
[73] Z. Wang and A. C. Bovik, "Mean squared error: Love it or leave it? A new look at signal fidelity measures," IEEE Signal Process. Mag. 26, 98-117 (2009).

[74] O. Demuynck and J. M. Menèndez, "Incoherent optical fiber bundle calibration for image transmission: faster, finer, and higher resolution image reconstruction," Opt. Eng. 50, 033601 (2011).

[75] Z. Wang, A. C. Bovik, H. R. Sheikh and E. P. Simoncelli, "Image quality assessment: From error visibility to structural similarity," IEEE Trans. on Image Process. 13, 600-612 (2004).

[76] Y. Choi, C. Yoon, M. Kim, T. D. Yang, C. Fang-Yen, R. R. Dasari, K. J. Lee, and W. Choi, "Scanner-free and wide-field endoscopic imaging by using a single multimode optical fiber," Phys. Rev. Lett. 109, 203901 (2012).

[77] T. P. Seward III, "Elongation and spheroidization of phase-separated particles in glass," J. Non-Cryst. Solids 15, 487-504 (1974).

[78] T. P. Seward III, "Some unusual optical properties of elongated phases in glasses," in The Physics of Non-Crystalline Solids, G. H. Frischat, ed. (Trans Tech Aedermannsdorf, Switzerland, 1977), pp. 342-347. 
Appendices 


\section{Appendix A}

\section{FD-BPM Codes}

\section{A.1 FD-BPM code in $\mathrm{C}$ language}

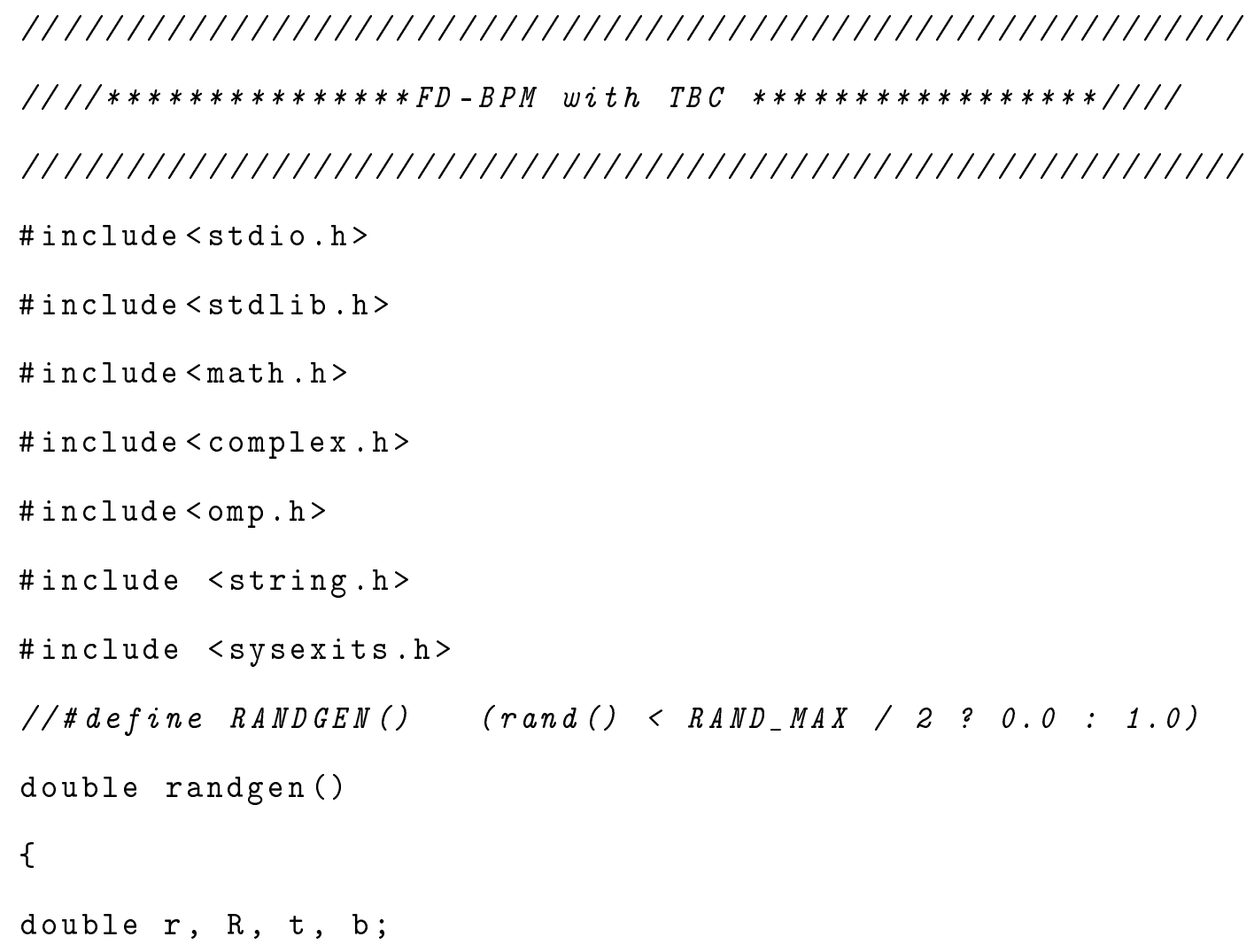




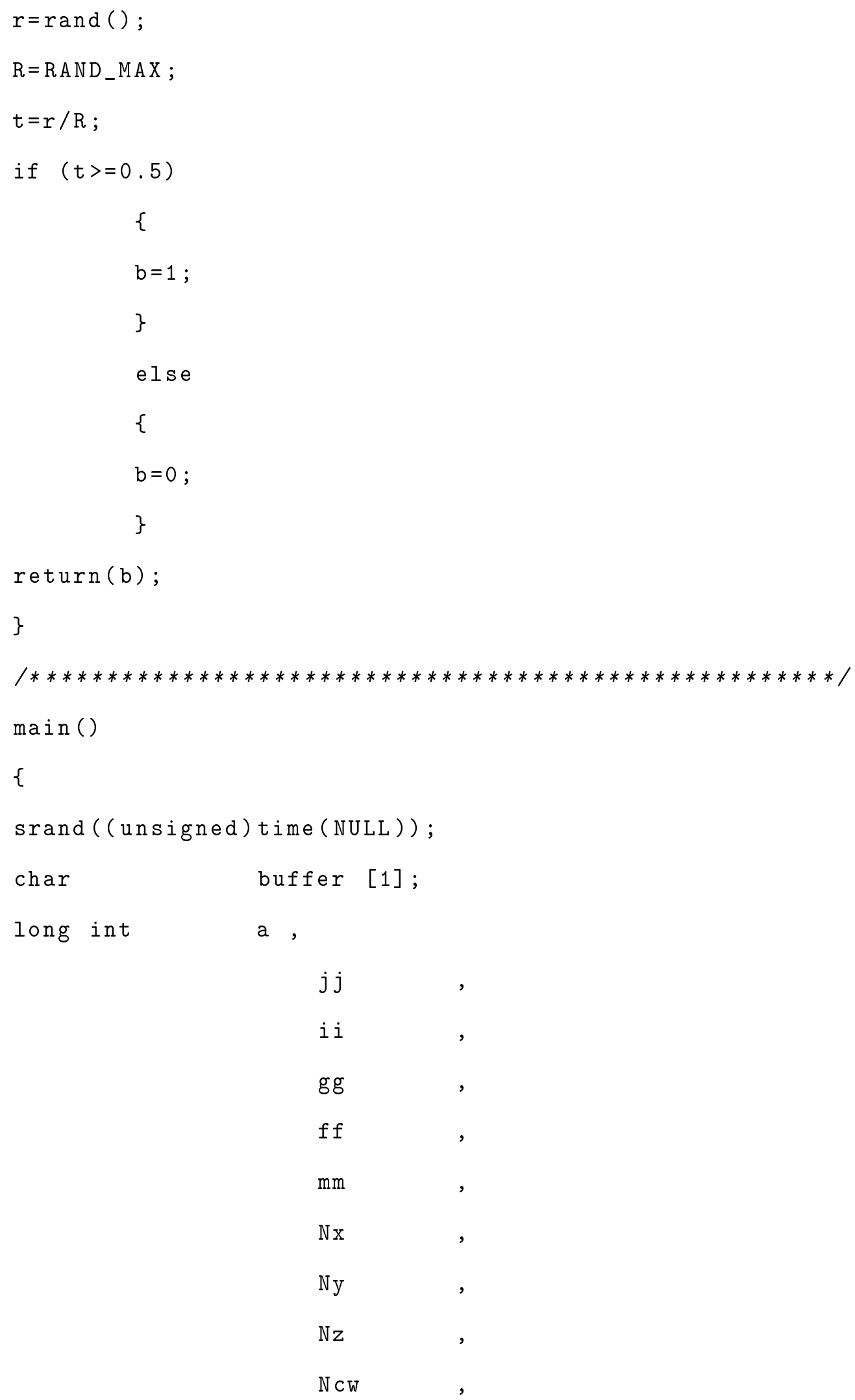




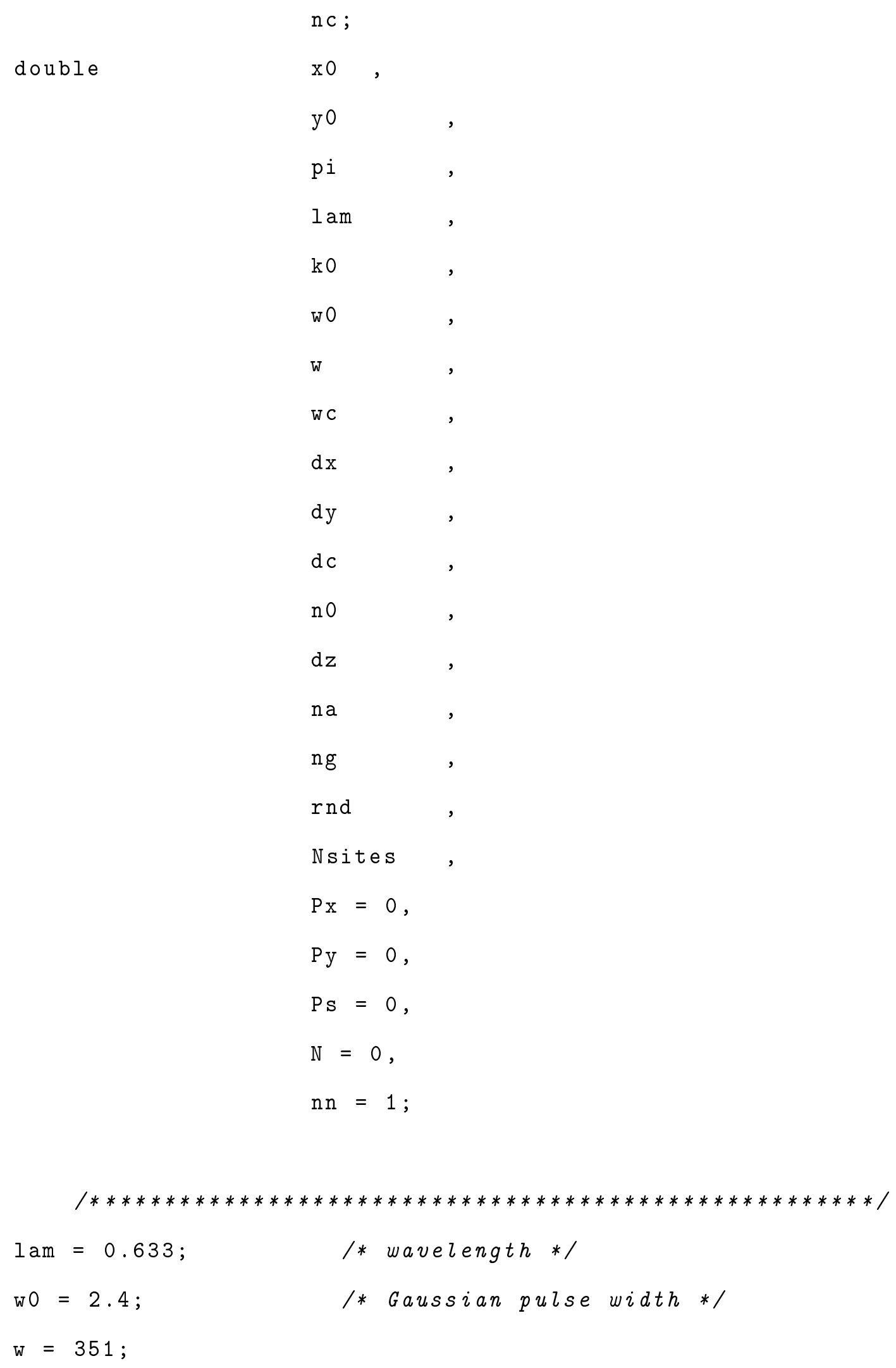




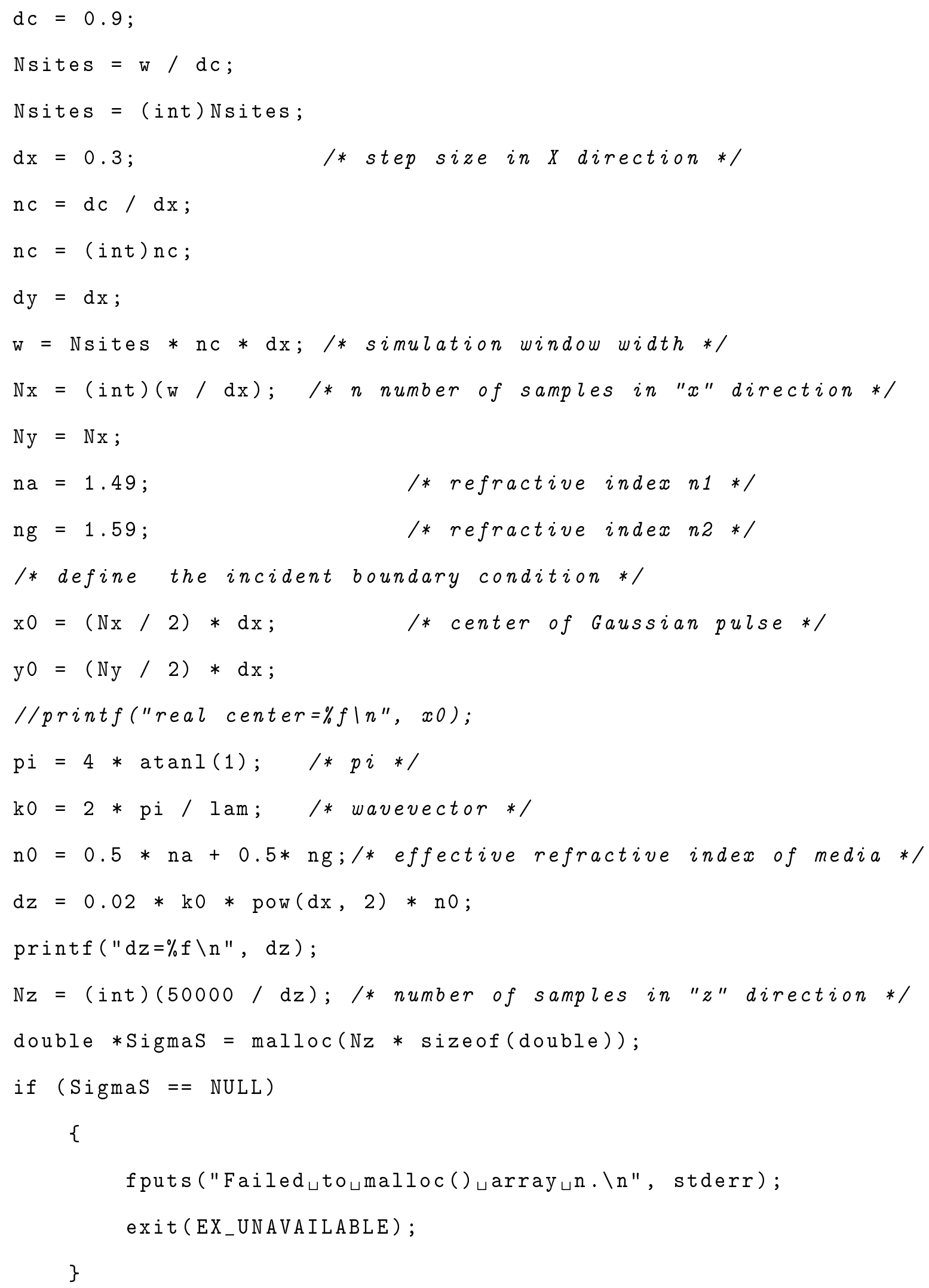




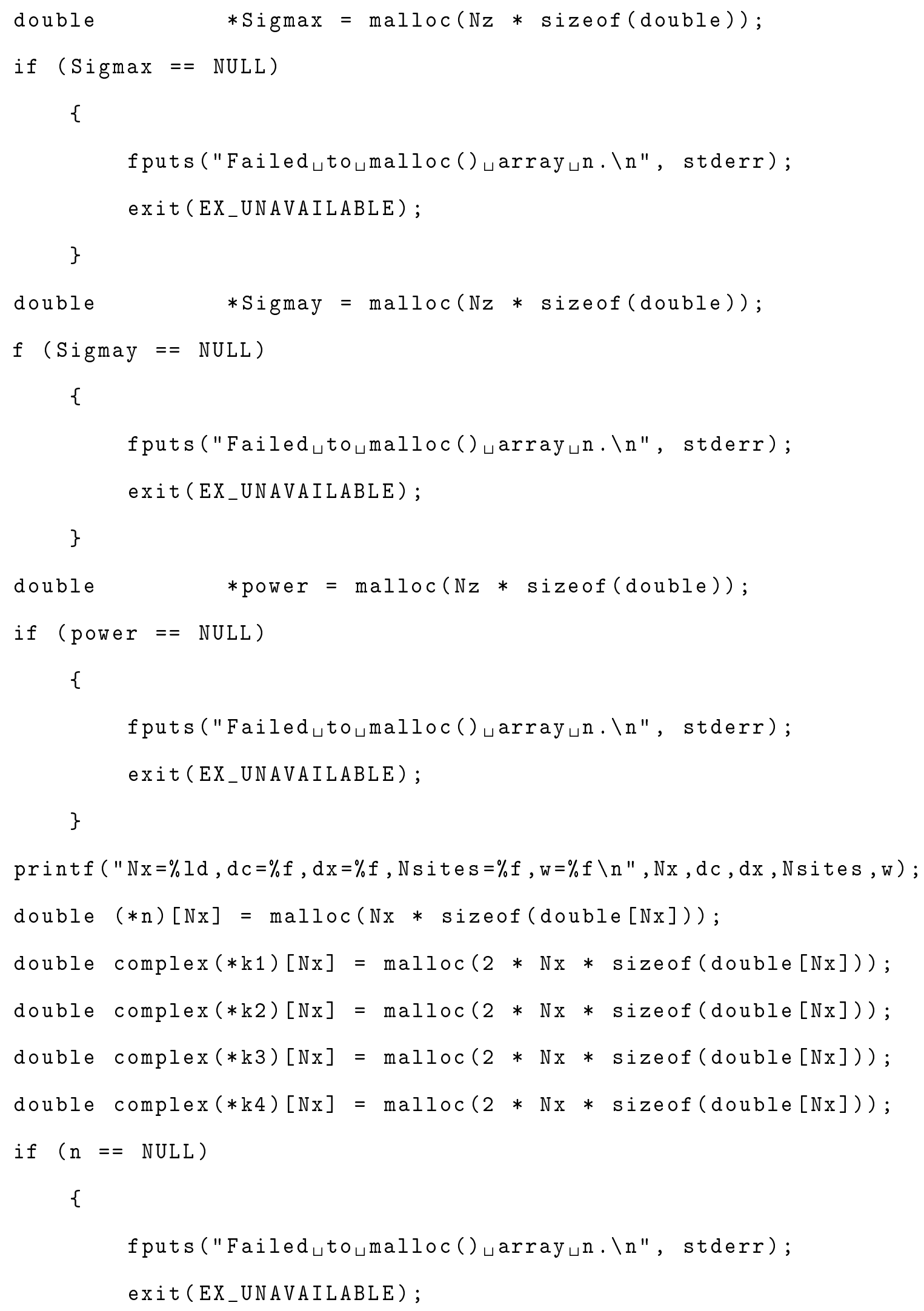




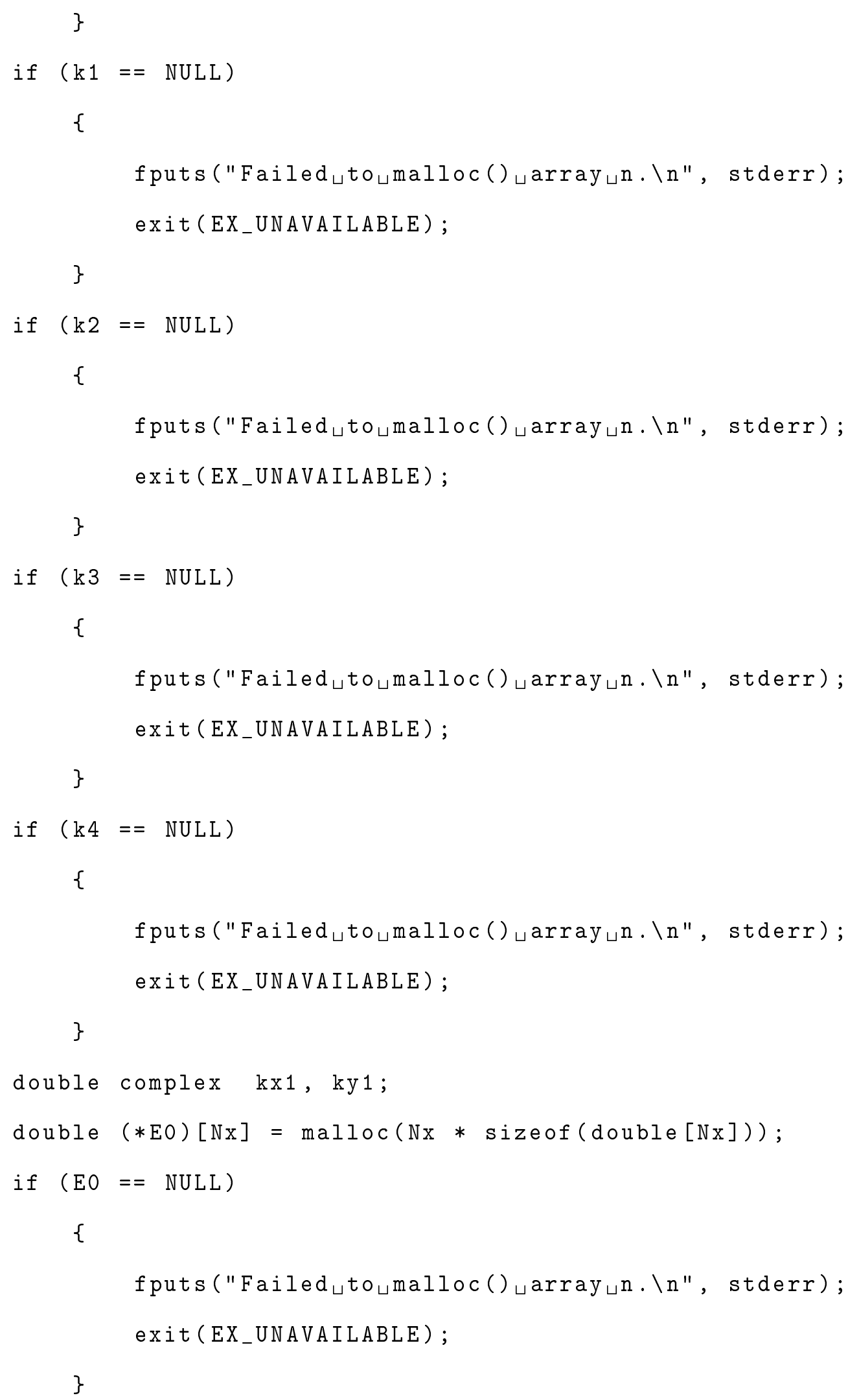




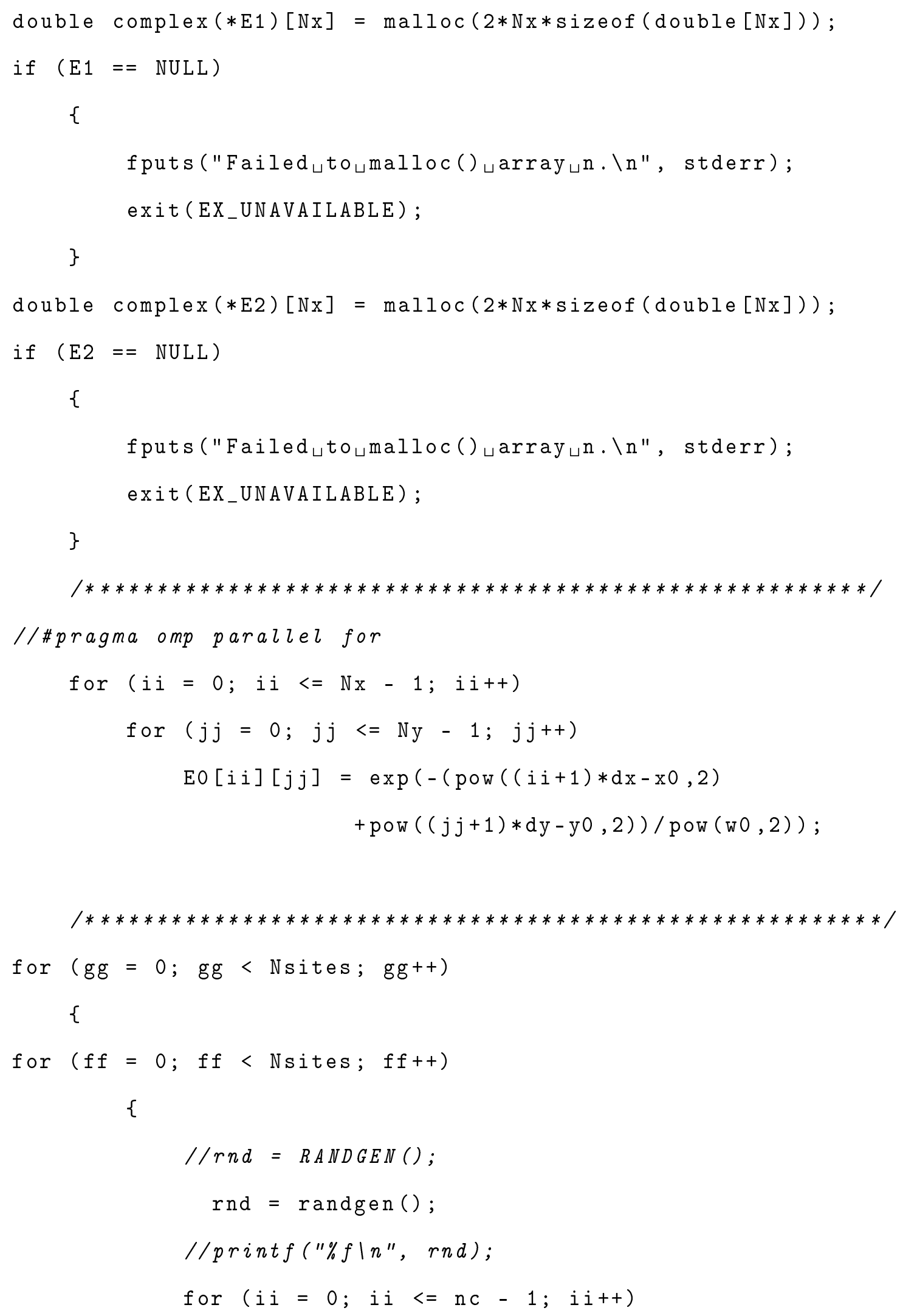




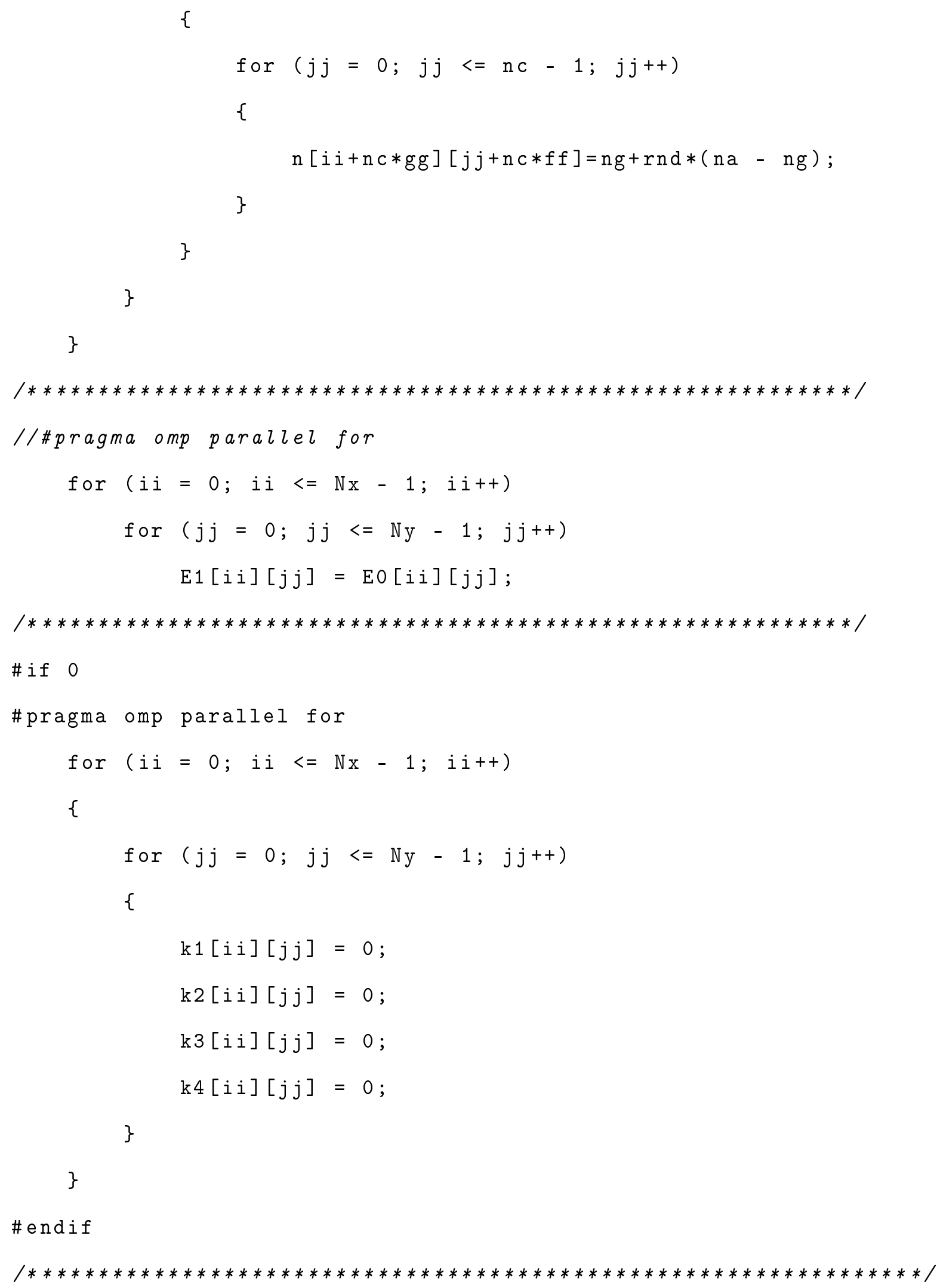


$/ * * * * * * * * * * * * * * * * * * * *$ Runge - Kutta method l $^{*} * * * * * * * * * * * * * * * * * /$

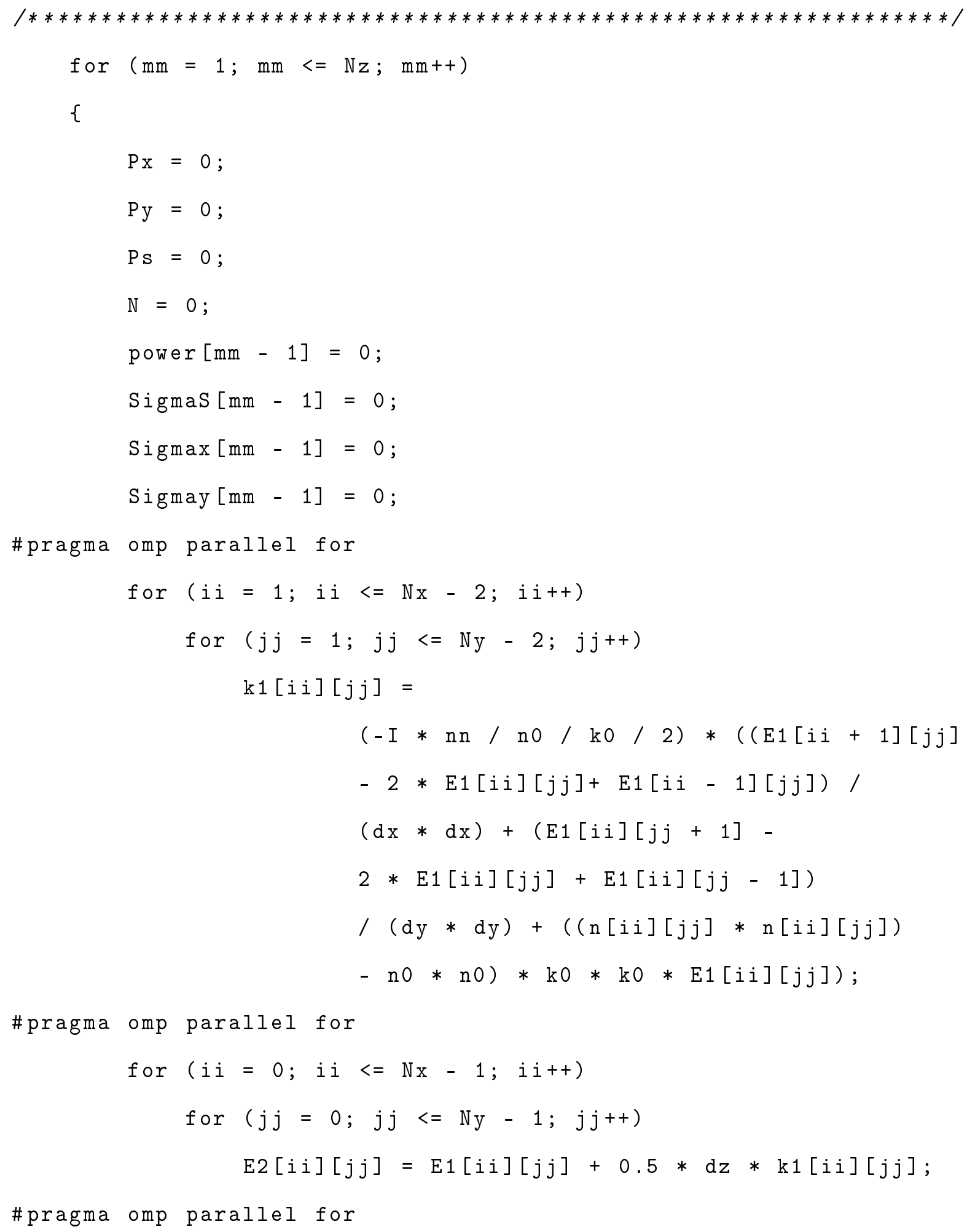




$$
\begin{aligned}
& \text { for }(i i=1 ; i i<=N x-2 ; i i++) \\
& \text { for }(j j=1 ; j j<=N y-2 ; j j++) \\
& \mathrm{k} 2[i i][j \mathrm{j}]=(-\mathrm{I} * \mathrm{nn} / \mathrm{no} / \mathrm{k} 0 / 2) * \\
& ((E 2[i i+1][j j]-2 * E 2[i i][j j] \\
& +E 2[i i-1][j j]) /(d x * d x) \\
& +(E 2[i i][j j+1]-2 * E 2[i i][j j] \\
& +E 2[i i][j j-1]) /(d y * d y) \\
& +(n[i i][j j] * n[i i][j j]-n 0 * n 0) \\
& \text { * } \mathrm{k} 0 * \mathrm{k} 0 * \mathrm{E} 2[i i][j j]) \text {; }
\end{aligned}
$$

\#pragma omp parallel for

$$
\begin{aligned}
\text { for } & (i i=0 ; i i<=N x-1 ; i i++) \\
\text { for } & (j j=0 ; j j<=N y-1 ; j j++) \\
& E 2[i i][j j]=E 1[i i][j j]+0.5 * d z * k 2[i i][j j] ;
\end{aligned}
$$

\#pragma omp parallel for

$$
\begin{aligned}
& / * * * * * * * * * * * * * * * * * * * * * * * * * * * * * * * * * * * * * * * * * * * * * * * / \\
& \text { for }(i i=1 ; i i<=N x-2 ; i i++) \\
& \text { for }(j j=1 ; j j<=N y-2 ; j j++) \\
& \mathrm{k} 3[\mathrm{i}][\mathrm{jj}]=(-\mathrm{I} * \mathrm{nn} / \mathrm{n} 0 / \mathrm{k} 0 / 2) * \\
& ((E 2[i i+1][j j]-2 * E 2[i i][j j] \\
& +E 2[i i-1][j j]) /(d x * d x) \\
& +(E 2[i i][j j+1]-2 * E 2[i i][j j] \\
& +E 2[i i][j j-1]) /(d y * d y) \\
& +(n[i i][j j] * n[i i][j j]-n 0 * n 0) \\
& * \mathrm{k} 0 * \mathrm{k} 0 * \mathrm{E} 2[i i][j j]) \text {; } \\
& \text { for }(i i=1 ; i i<=N x-2 ; i i++) \\
& \text { for }(j j=1 ; j j<=N y-2 ; j j++) \\
& \mathrm{E} 2[\mathrm{i} i][j \mathrm{j}]=\mathrm{E} 1[\mathrm{i} i][\mathrm{j} j]+\mathrm{dz} * \mathrm{k} 3[\mathrm{ii}][\mathrm{j}] \text {; }
\end{aligned}
$$

\#pragma omp parallel for 


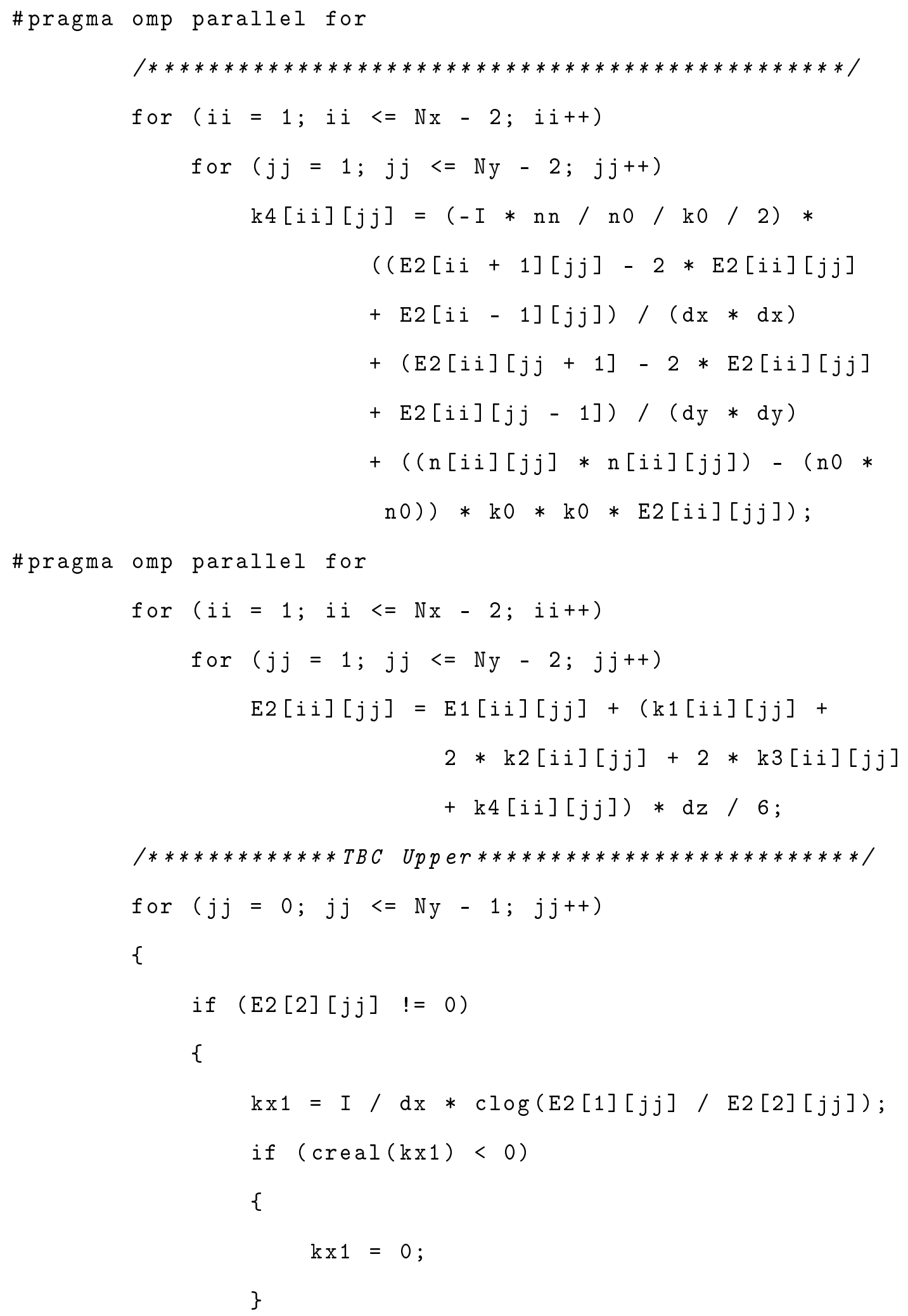




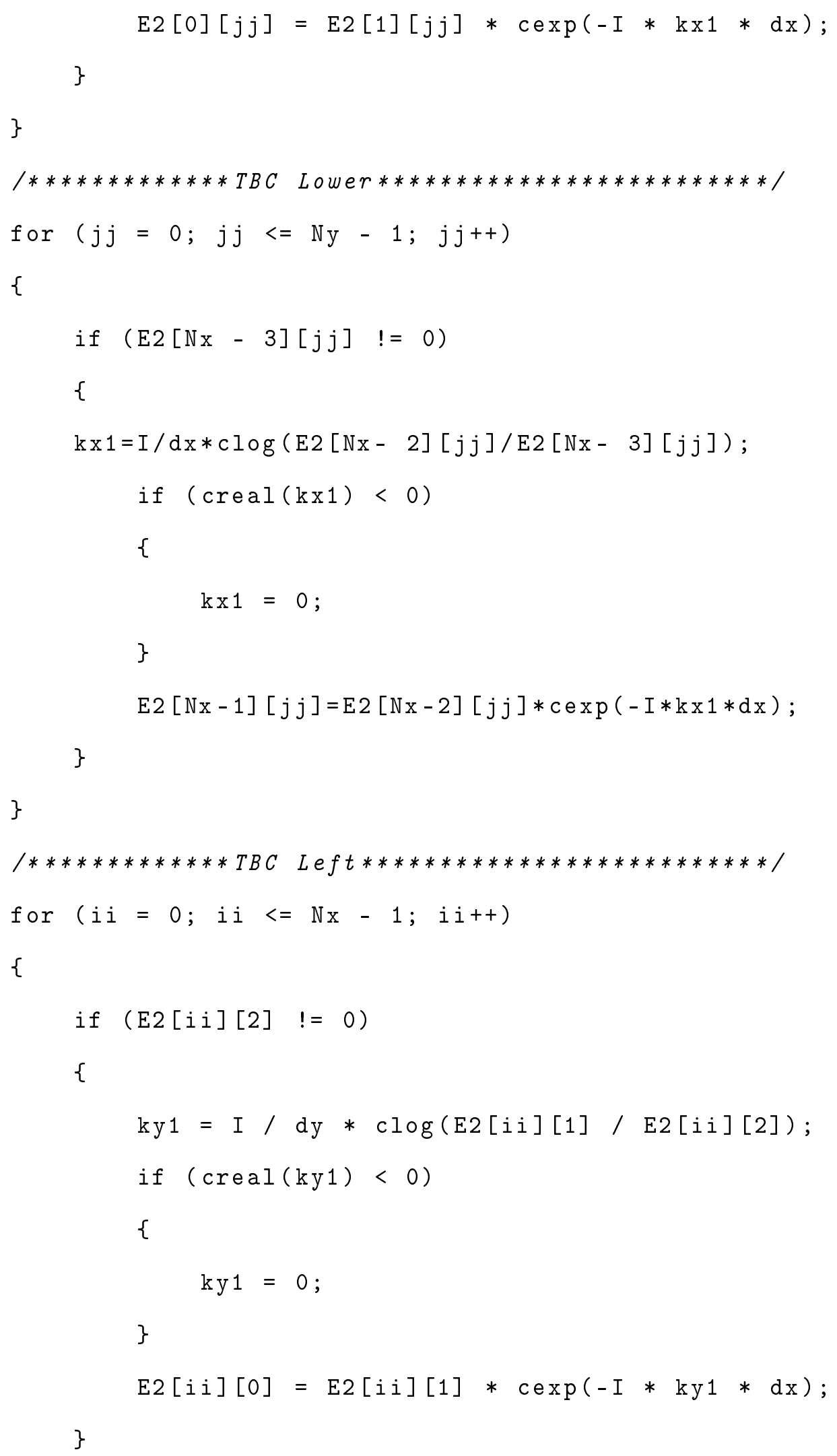




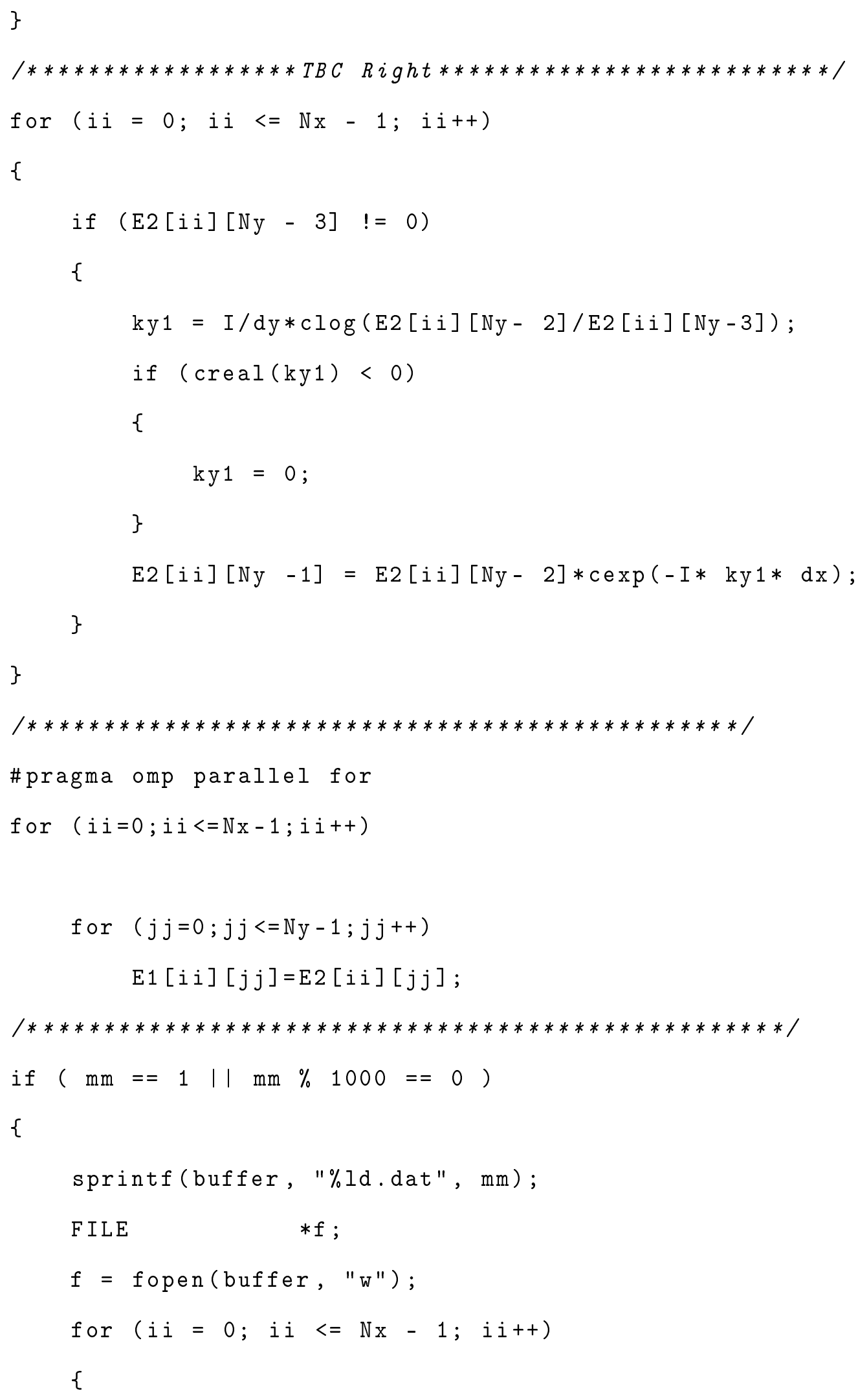




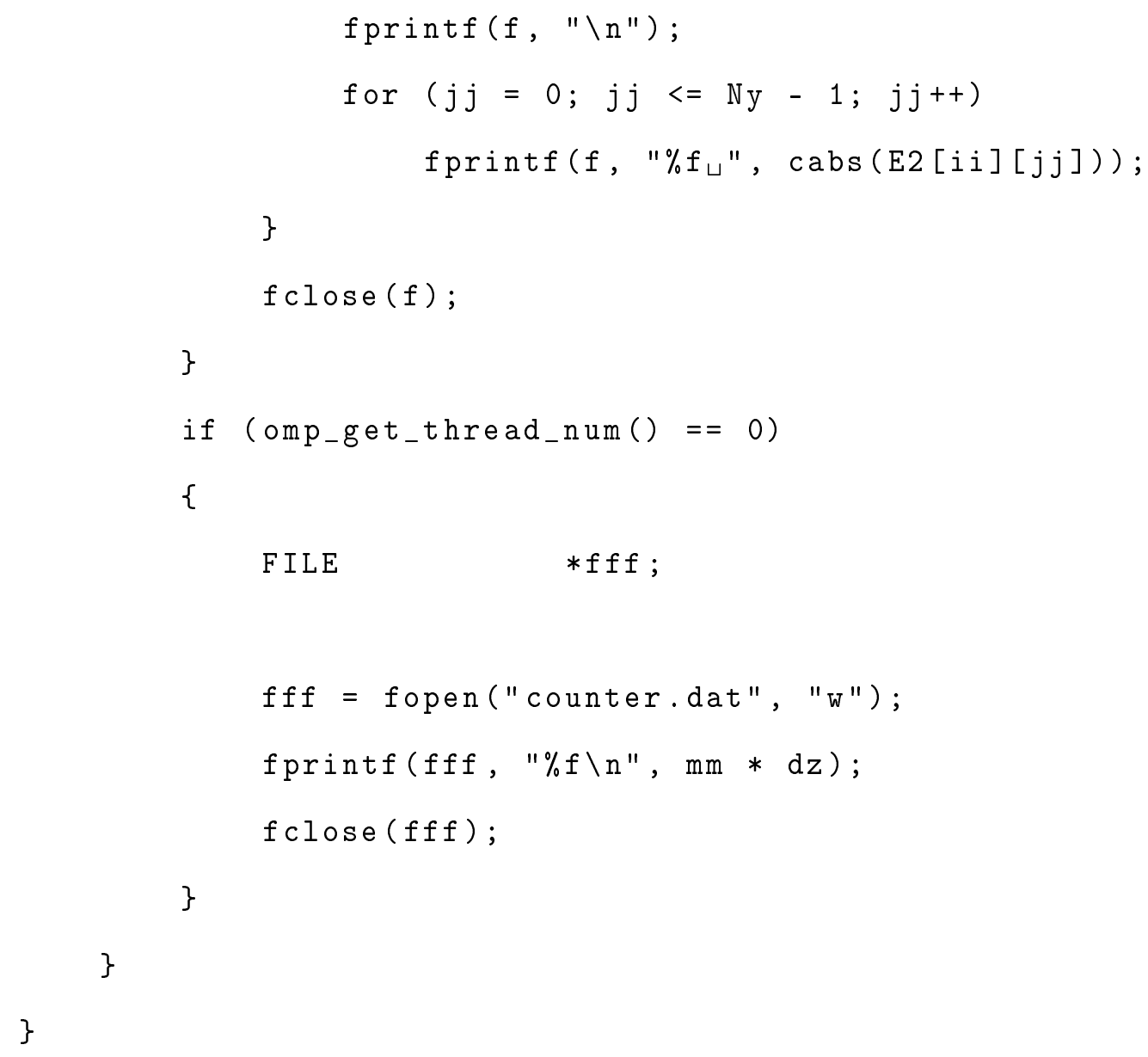

\section{A.2 FD-BPM code in Matlab}

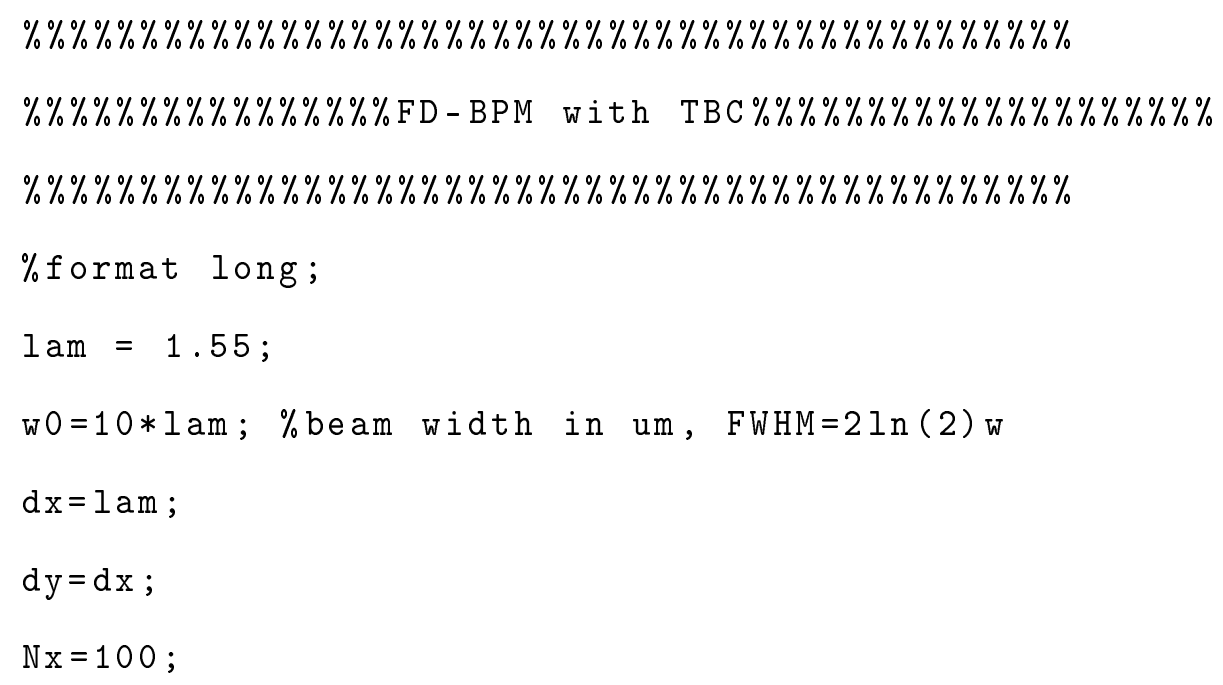




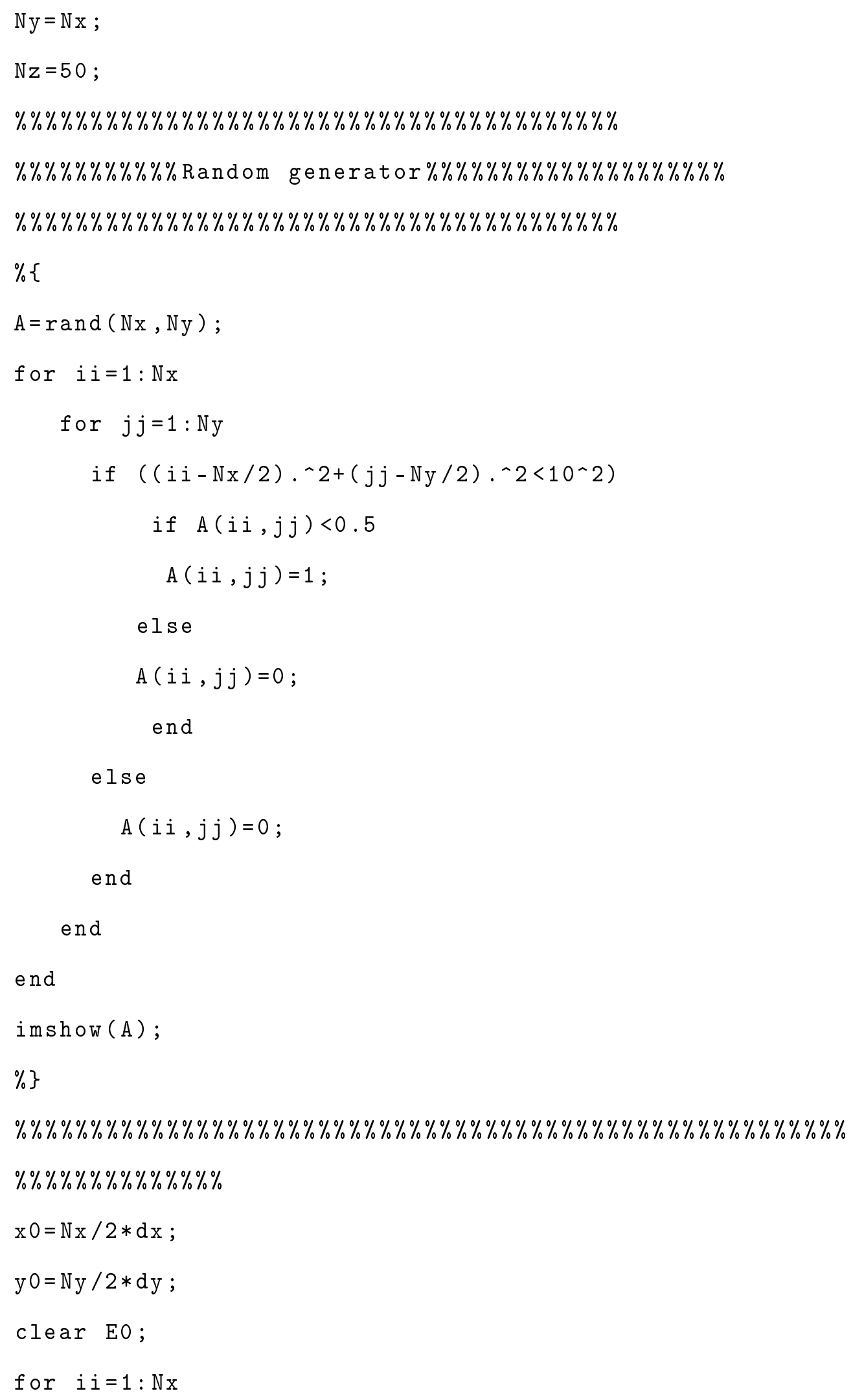




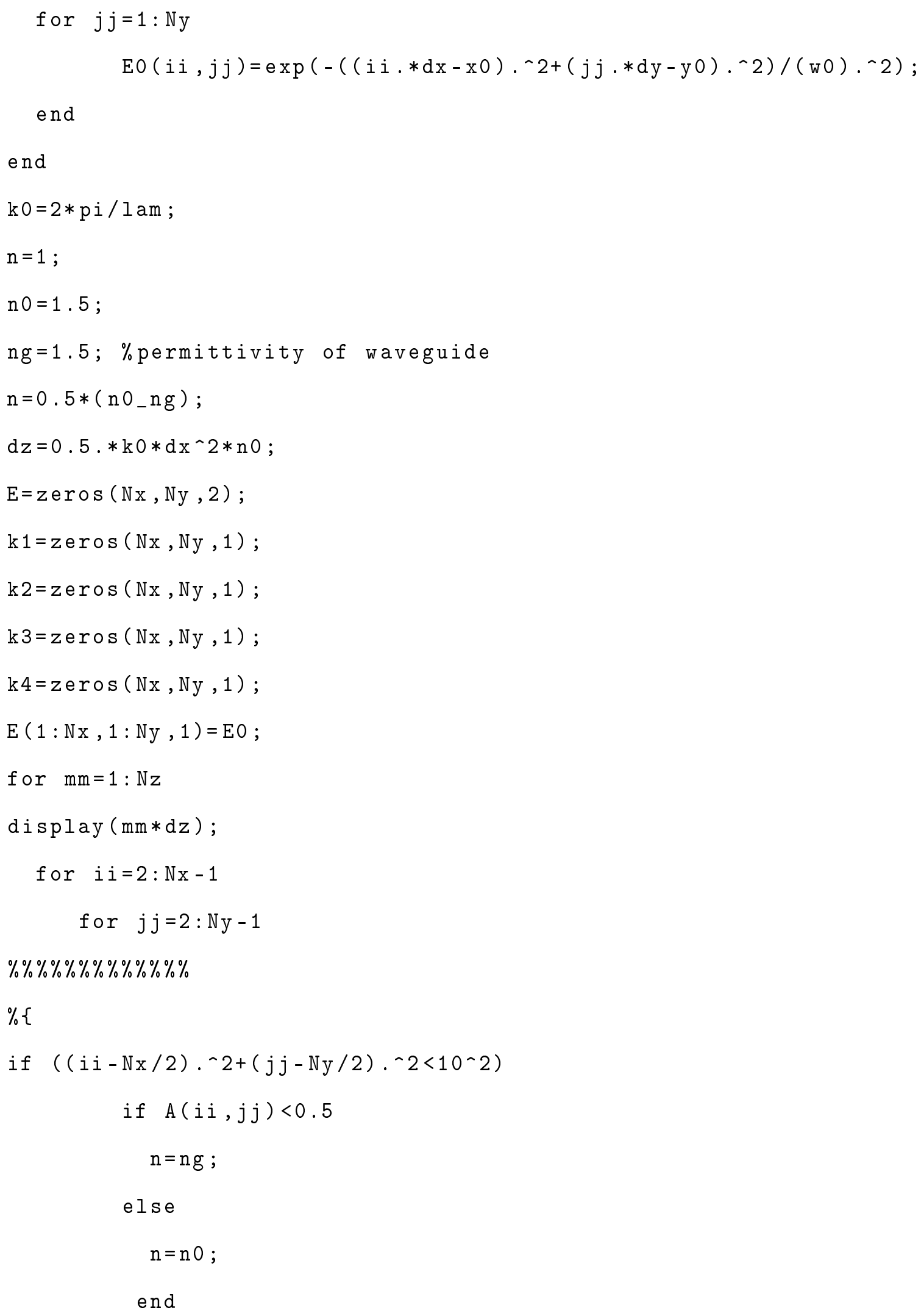


else
$n=n O ;$

end

$\%\}$

$\% \% \% \% \% \% \% \% \% \% \% \%$

$\mathrm{k} 1(\mathrm{i} i, j \mathrm{j})=(-\mathrm{j} / 2 / \mathrm{n} 0 / \mathrm{k} 0) *((\mathrm{E}(\mathrm{i} i+1, j \mathrm{j}, 1)$ $-2 * E(i i, j j, 1)+E(i i-1, j j, 1)) . / d x^{\wedge} 2$ $+(E(i i, j j+1,1)-2 * E(i i, j j, 1)+$ $E(i i, j j-1,1)) . / d y^{\wedge} 2+$ $(n . \wedge 2-n 0 . \wedge 2) . * k 0 \wedge 2 . * E(i i, j j, 1))$;

end

end

$\mathrm{E}(:,:, 2)=\mathrm{E}(:,:, 1)+0.5 * \mathrm{dz} . * \mathrm{k} 1 ;$

for $i i=2: N x-1$

for $j \mathrm{j}=2: \mathrm{Ny}-1$

$\% \% \% \% \% \% \% \% \% \%$

$\%\{$

if $\left((i i-N x / 2) \cdot \wedge 2+(j j-N y / 2) \cdot \wedge 2<10^{\wedge} 2\right)$

if $A(i i, j j)<0.5$

$\mathrm{n}=\mathrm{ng}$;

else

$\mathrm{n}=\mathrm{n} 0$;

end

else

$\mathrm{n}=\mathrm{n} 0$;

end

$\%\}$

$\% \% \% \% \% \% \% \% \% \% \% \%$ 


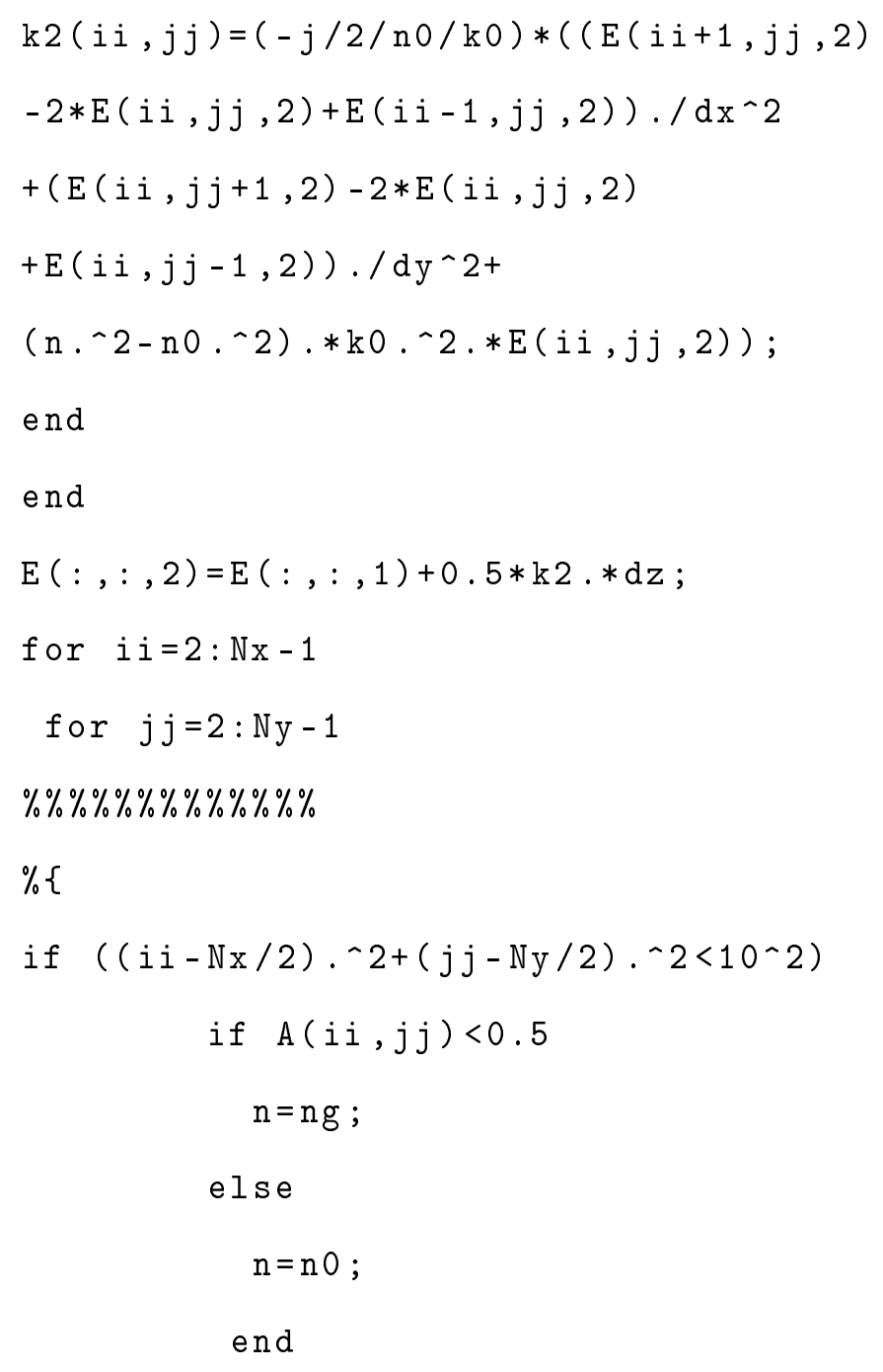




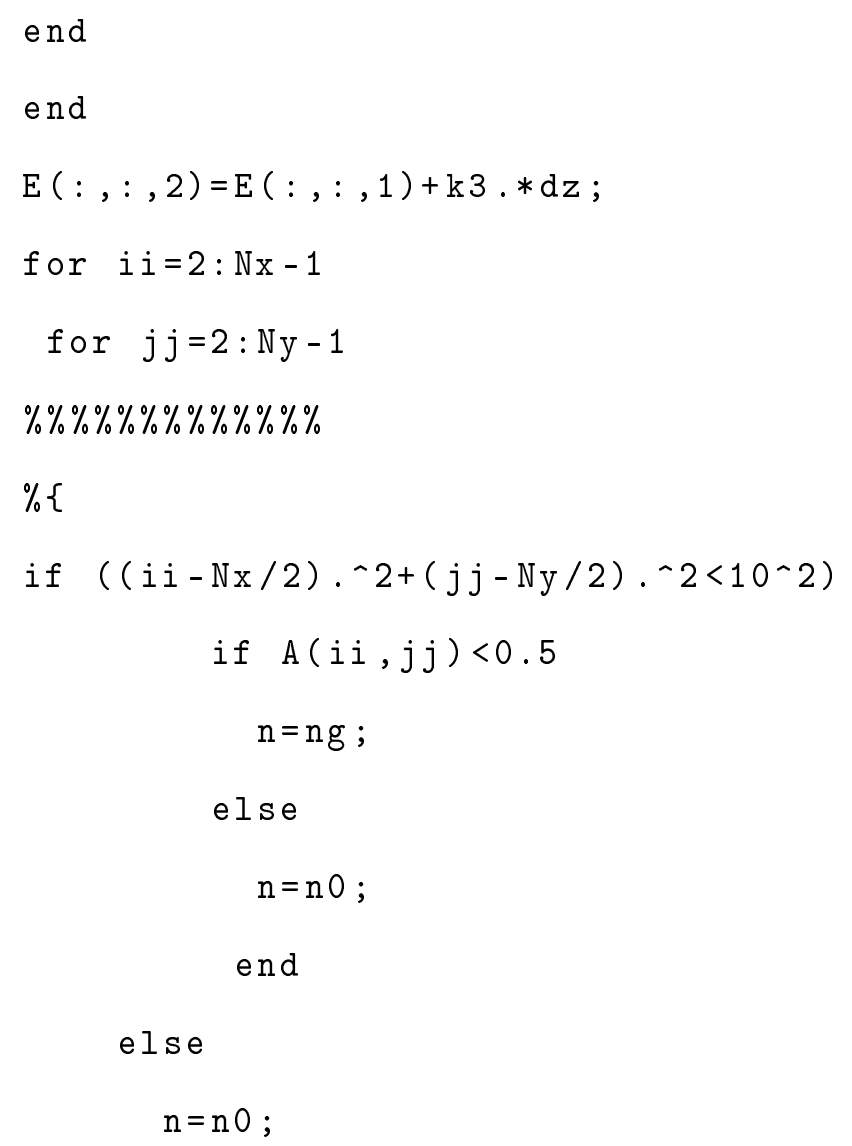


$\% \% \% \% \% \% \% \% \% \% \% \% \% \%$ TBC Right boundary $\% \% \% \% \% \% \% \% \% \% \% \% \% \% \% \% \% \%$ $\mathrm{kr} 1=\mathrm{j} / \operatorname{sqrt}(2) . / \mathrm{dx} \cdot * \log (E(N x-1,:, 2) . / E(N x-2,:, 2))$;

$E(N x,:, 2)=E(N x-1,:, 2) . * \exp (-j * \operatorname{kr} 1 * d x * \operatorname{sqrt}(2)) ;$

$\% \% \% \% \% \% \% \% \% \% \% \% \%$ TBC Left boundary $\% \% \% \% \% \% \% \% \% \% \% \% \% \% \%$

$\operatorname{kr} 2=j / \operatorname{sqrt}(2) . / d x \cdot * \log (E(2,:, 2) . / E(3,:, 2))$;

$E(1,:, 2)=E(2,:, 2) . * \exp (-j * \operatorname{kr} 2 * d x * \operatorname{sqrt}(2))$;

$\% \% \% \% \% \% \% \% \% \% \% \% \% \%$ TBC Upper boundary $\% \% \% \% \% \% \% \% \% \% \% \% \% \% \% \% \%$ $\operatorname{kr} 3=j . / d x / \operatorname{sqrt}(2) . * \log (E(:, 2,2) . / E(:, 3,2))$;

$\%$ display $(\operatorname{abs}(E(:, 2, \mathrm{~mm})))$;

$E(:, 1,2)=E(:, 2,2) . * \exp (-j * \operatorname{kr} 3 * \operatorname{sqrt}(2) * d x)$;

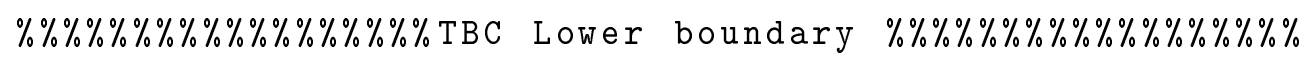

$\mathrm{kr} 4=\mathrm{j} . / \mathrm{dx} / \operatorname{sqrt}(2) . * \log (\mathrm{E}(:, \mathrm{Ny}-1,2) . / E(:, N y-2,2))$;

$E(:, N y, 2)=E(:, N y-1,2) . * \exp (-j * k r 4 * \operatorname{sqrt}(2) * d x)$;

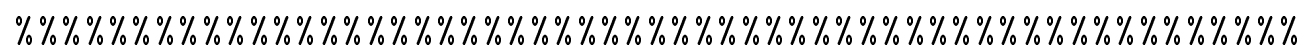

$E(:,:, 1)=E(:,:, 2)$;

end

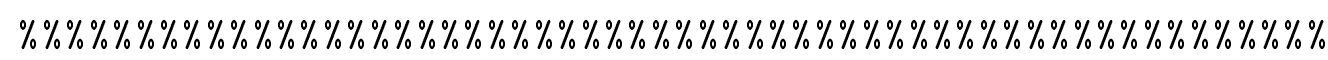
$\operatorname{surf}(\operatorname{abs}(E(:,:, 2)))$;

shading interp; 


\title{
Appendix B
}

\section{Shell scripting for compiling and submitting the jobs}

\author{
B.1 Building a job on Peregrine \\ $\# ! / \mathrm{b}$ in $/ \mathrm{sh}$ \\ \#PBS -o build-output.txt \\ \#PBS -e build-errors.txt \\ cd \$PBS_0_WORKDIR \\ export CFLAGS="-I/usr/local/include $\sqcup-02$ " \\ export LDFLAGS="-L/usr/local/libu-lcomplex" \\ export $\mathrm{CC}=\mathrm{gcc} 46$ \\ make
}




\section{B.2 Submitting a job of Peregrine}

\#!/bin/bash

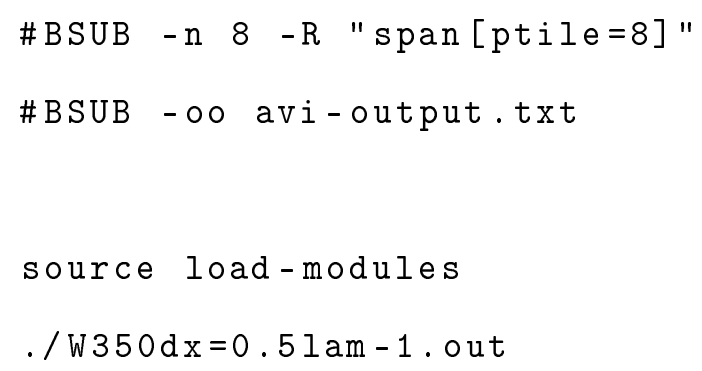

\section{B.3 Compiling multiple jobs on AVI}

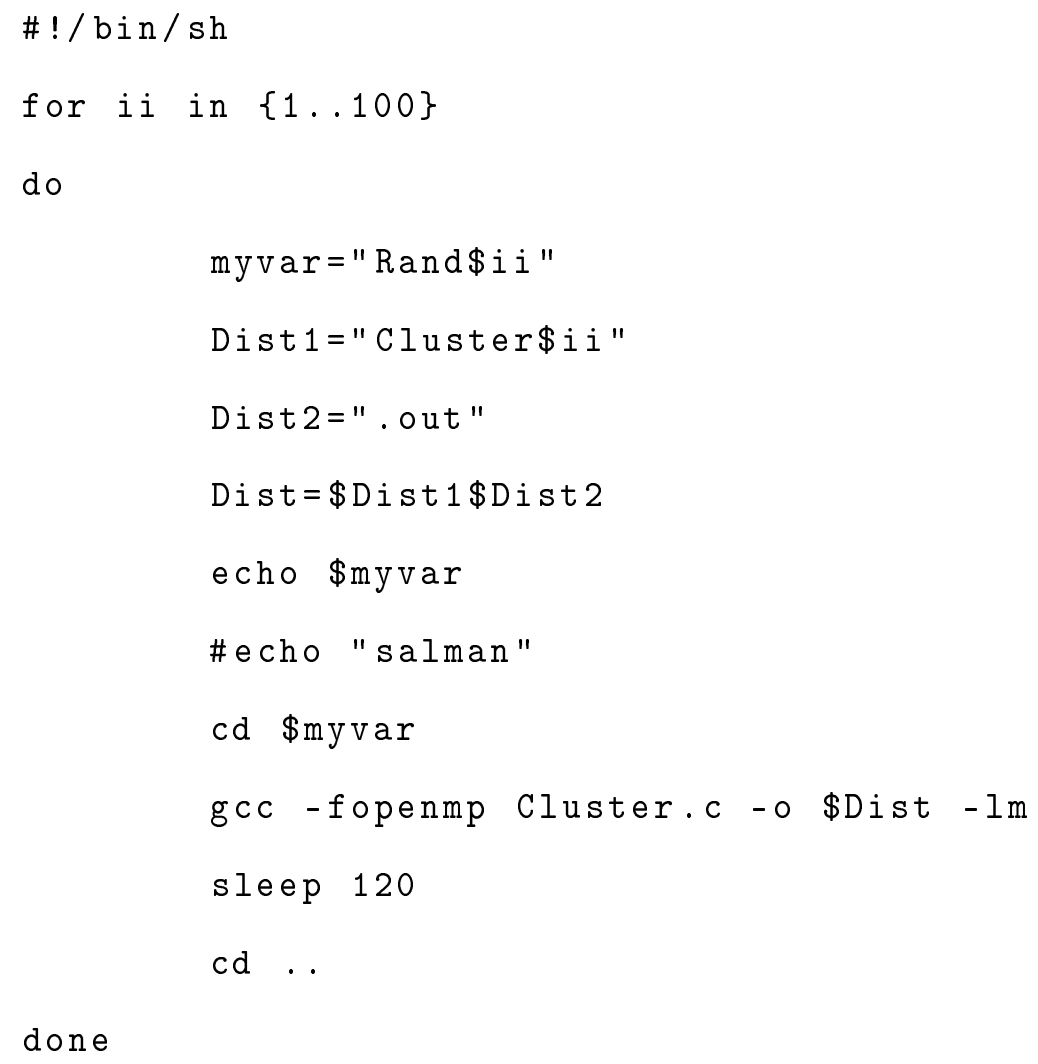




\section{B.4 Submitting multiple jobs on AVI}

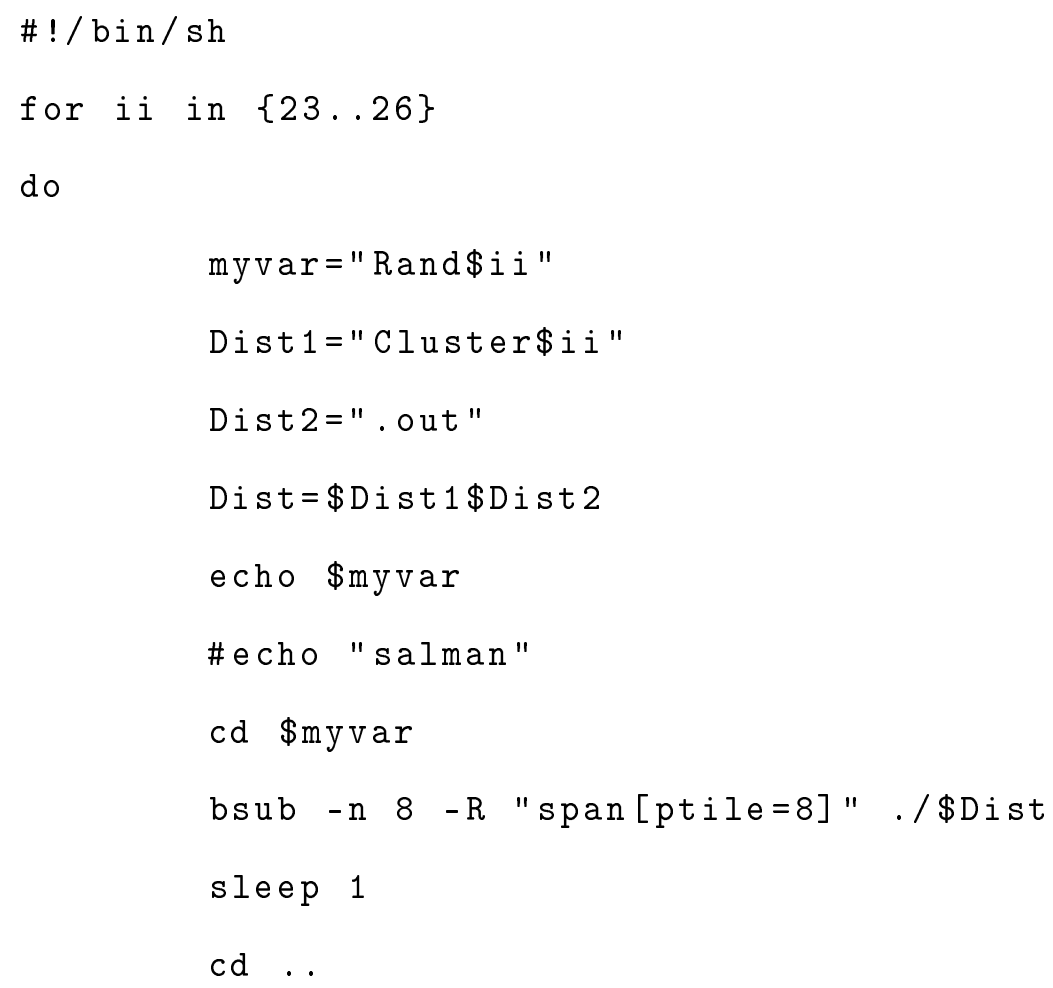




\section{Appendix C}

\section{Stability analysis for $2^{\text {nd }}$ order Runge-Kutta method}

In here, the stability analysis for the $2^{\text {nd }}$ order ordinary differential equation is carried out. The paraxial wave equation using Runge-Kutta method can be written as

$$
A^{m+1}=A^{m}+0.5\left(K_{1}+K_{2}\right) ;
$$

where $k_{1}, k_{2}$ are as follows:

$$
\begin{aligned}
& K 1=\frac{-j}{2 n_{0} k_{0}}\left[\frac{A_{i+1}^{m}-2 A_{i}^{m}+A_{i-1}^{m}}{\Delta x^{2}}+\left(k^{2}-k_{0}^{2}\right) A_{i}^{m}\right], \\
& K_{2}=\frac{-j}{2 n_{0} k_{0}}\left[\frac{A_{i+1}^{m}-2 A_{i}^{m}+A_{i-1}^{m}}{\Delta x^{2}}+\left(k^{2}-k_{0}^{2}\right)\left(A_{i}^{m}+K_{1} \Delta z\right)\right], \\
& A^{m+1}=A^{m}+0.5\left(K_{1}+K_{2}\right) \Delta z
\end{aligned}
$$


If $A(x, z)=A_{i}^{m}=A^{m} e^{j t i \Delta x}$ where $t$ is an arbitrary wave vector:

$$
\begin{aligned}
& K 1=\frac{-j}{2 n_{0} k_{0}} A^{m} e^{j t i \Delta x}\left[\frac{e^{j t \Delta x}-2+e^{-j t \Delta x}}{\Delta x^{2}}+\left(k^{2}-k_{0}^{2}\right)\right], \\
& K_{2}=\frac{-j}{2 n_{0} k_{0}}\left[e^{j t i \Delta x} A^{m} \frac{e^{j t \Delta x}-2+e^{-j t \Delta x}}{\Delta x^{2}}+\left(k^{2}-k_{0}^{2}\right)\left(A^{m} e^{j t i \Delta x}+K_{1} \Delta z\right)\right], \\
& A^{m+1}=A^{m}+0.5\left(K_{1}+K_{2}\right) \Delta z
\end{aligned}
$$

hence,

$$
\begin{aligned}
& A^{m+1}=A^{m}+0.5\left(K_{1}+K_{2}\right) \Delta z \\
& A^{m+1}=A^{m}+0.5 \Delta z A^{m} \frac{-j}{2 n_{0} k_{0}}\left\{\left[\frac{e^{j t \Delta x}-2+e^{-j t \Delta x}}{\Delta x^{2}}+\left(k^{2}-k_{0}^{2}\right)\right]+\ldots\right. \\
& \left.\left[\frac{e^{j t \Delta x}-2+e^{-j t \Delta x}}{\Delta x^{2}}+\left(k^{2}-k_{0}^{2}\right)\left(1+\Delta z\left[\frac{e^{j t \Delta x}-2+e^{-j t \Delta x}}{\Delta x^{2}}+\left(k^{2}-k_{0}^{2}\right)\right]\right)\right]\right\}
\end{aligned}
$$

Or using the $H$ parameter:

$$
\frac{\partial A}{\partial z}=-j H A
$$

where $H$ is

$$
H=\frac{1}{2 k}\left[\frac{\partial^{2}}{\partial x^{2}}+\left(k^{2}-k_{0}^{2}\right)\right]
$$


so $K_{1}$ and $K_{2}$ in the $2^{\text {nd }}$ Runge-Kutta method are:

$$
\begin{aligned}
& K_{1}=\Delta z\left(-j H A^{m}\right) \\
& K_{2}=\Delta z\left(-j H\left(A^{m}+0.5 K_{1}\right)\right)=-j \Delta z H\left(A^{m}-j 0.5 H \Delta z A^{m}\right) \\
& A^{m+1}=A^{m}+K_{2} \\
& \left.A^{m+1}=A^{m}-j \Delta z H A^{m}-0.5 H^{2} \Delta z^{2} A^{m}\right)
\end{aligned}
$$

So

$$
\left|\frac{A^{m+1}}{A^{m}}\right|=\left|1-j \Delta z H-0.5 H^{2} \Delta z^{2}\right|=\sqrt{\left(1-0.5 H^{2} \Delta z^{2}\right)^{2}+\Delta z^{2} H^{2}}
$$

then,

$$
\left|\frac{A^{m+1}}{A^{m}}\right|=\sqrt{1+0.25 H^{4} \Delta z^{4}}>1
$$

The gain factor shows that the $2^{\text {nd }}$ order Runge-Kutta method is unconditionally unstable. 


\section{CURRICULUM VITAE}

\section{Salman Karbasi}

\section{RESEARCH INTERESTS}

- Electromagnetic wave propagation

- Numerical analysis

- Design and development of optical devices

- Image transport

\section{EDUCATION}

- Doctor of Philosophy, Electrical Engineering, $\quad$ Sep. 2009 - Jan. 2014 University of Wisconsin-Milwaukee, Milwaukee, WI, USA.

- Thesis title: Transverse Anderson localization in disordered optical fibers

- Advisor: Prof. Arash Mafi

- Master of Science, Electrical Engineering, $\quad$ Sep. 2006 - Dec. 2008 Tarbiat Modares University, Tehran, Iran.

- Thesis title: Design and optimization of crescent shape surface plasmon resonance nano-Antenna

- Advisor: Prof. Keyvan Forooraghi

- Bachelor of Science, Electrical Engineering, $\quad$ Sep. 2002 - Sep. 2006 Yazd University, Yazd, Iran.

- Thesis title: Design and implement of a rescue robot: recognizer and sorter of objects using 3D image processing

- Advisor: Prof. M. T. Sadeghi 